Portland State University

PDXScholar

Summer 10-8-2014

\title{
SLM-based Fourier Differential Interference Contrast Microscopy
}

Sahand Noorizadeh

Portland State University

Follow this and additional works at: https://pdxscholar.library.pdx.edu/open_access_etds

Part of the Electrical and Computer Engineering Commons, and the Optics Commons Let us know how access to this document benefits you.

\section{Recommended Citation}

Noorizadeh, Sahand, "SLM-based Fourier Differential Interference Contrast Microscopy" (2014).

Dissertations and Theses. Paper 2011.

https://doi.org/10.15760/etd.2010

This Thesis is brought to you for free and open access. It has been accepted for inclusion in Dissertations and Theses by an authorized administrator of PDXScholar. Please contact us if we can make this document more accessible: pdxscholar@pdx.edu. 
SLM-based Fourier Differential Interference Contrast Microscopy

by

Sahand Noorizadeh

A thesis submitted in partial fulfillment of the requirements for the degree of

Master of Science

in

Electrical and Computer Engineering

Thesis Committee:

Donald Duncan

Erik Sánchez

James McNames

Portland State University

2014 


\begin{abstract}
Optical phase microscopy provides a view of objects that have minimal to no effect on the detected intensity of light that are unobservable by standard microscopy techniques. Since its inception just over 60 years ago that gave us a vision to an unseen world and earned Frits Zernike the Nobel prize in physics in 1953, phase microscopy has evolved to find various applications in biological cell imaging, crystallography, semiconductor failure analysis, and more. Two common and commercially available techniques are phase contrast and differential interference contrast (DIC). In phase contrast method, a large portion of the unscattered light that accounts for the majority of the light passing unaffected through a transparent medium is blocked to allow the scattered light due to the object to be observed with higher contrast. DIC is a self-referenced interferometer that transduces phase variation to intensity variation. While being established as fundamental tools in many scientific and engineering disciplines, the traditional implementation of these techniques lacks the ability to provide the means for quantitative and repeatable measurement without an extensive and cumbersome calibration. The rapidly growing fields in modern biology meteorology and nano-technology have emphasized the demand for a more robust and convenient quantitative phase microscopy.
\end{abstract}

The recent emergence of modern optical devices such as high resolution programmable spatial light modulators (SLM) has enabled a multitude of research activities over the past decade to reinvent phase microscopy in unconventional ways. This work is concerned with an implementation of a DIC microscope containing a 4-f system at its core with a programmable SLM placed at the frequency plane of the imaging system that allows for employing Fourier pair transforms for wavefront manipulation. This configuration of microscope provides a convenient way to per- 
form both wavefront shearing with quantifiable arbitrary shear amount and direction as well as phase stepping interferometry by programming the SLM with a series of numerically generated patterns and digitally capturing interferograms for each step which are then used to calculate the objects phase gradient map. Wavefront shearing is performed by generating a pattern for the SLM where two phase ramp patterns with opposite slopes are interleaved through a random selection process with uniform distribution in order to mimic the simultaneous presence of the ramps on the same plane. The theoretical treatment accompanied by simulations and experimental results and discussion are presented in this work. 


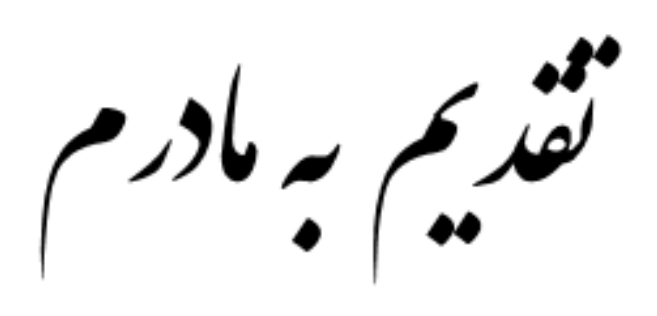

To Mom 


\section{Acknowledgments}

First and foremost, I would like to thank my adviser Dr. Donald D. Duncan for his patience, guidance, and teachings over the past three years and also for personally funding a large portion of this research work. My immense gratitude is extended to Dr. Steven Jacques of Biomedical Engineering department at Oregon Health and Science University for making their brand new SLM available to us. Clearly, without it this work would not have been possible. Dr. Scott Prahl of Oregon Institute of Technology played an instrumental role in getting the experiments started and training me with the basic practices of optics lab experiments. He also made some key materials available from his lab for the experiments. I benefited a great deal from the guidance and mentorship of my friend and peer Jimmy Gladish. His hard-earned experience in building optical systems was an invaluable asset for this project.

I am grateful beyond measure to Mr. Amir Aghdaei and Marcus da Silva of Tektronix for putting their trust and faith in me and helping me obtain education financial assistance for my graduate studies. Accomplishing my degree along with a full-time job would have been impossible without the kind and caring understanding of my managers Thomas Freni, Steve Curtis, and Anthony Woods that allowed me to have flexible time when needed over the past three years. I strive to make this a valuable investment on their part

And last but not least, Dr. John A. Buck and Dr. Paul Steffes of Georgia Institute of Technology have been role models in my academic and professional life. The excitement and inspiration that I got from working with them in their labs during my undergraduate years is still strong within me, thanks to them. 


\section{Contents}

1 Introduction 1

1.1 Phase Microscopy . . . . . . . . . . . . . . . . . . . . . . . 2

1.2 Survey of Recent Literature . . . . . . . . . . . . . . . . 6

1.2.1 Differential Interference Contrast Microscopy with SLM . . . . 6

1.2.2 Spiral Phase Microscopy . . . . . . . . . . . . . . . . . . 10

1.3 A Look Ahead . . . . . . . . . . . . . . . . . . . 14

2 Fourier DIC Microscopy: Theory 18

2.1 Wavefront Distortion . . . . . . . . . . . . . . . 19

2.2 Principles of Differential Interference Contrast . . . . . . . . . . . . . 22

2.3 Wavefront Modulation with a 4-f System . . . . . . . . . . . 26

2.4 Wavefront Shearing with a 4 -f System . . . . . . . . . . . . 28

2.5 Phase Pattern Combination by Random Selection . . . . . . . . . . . 33 
2.6 Phase Shifting Interferometry with a 4 -f System . . . . . . . . . . .

3 Numerical Simulations and Analysis $\quad 38$

3.1 Simulation of the 4 -f System and PSI . . . . . . . . . . . . . . 39

3.2 Simulation of the Phase Object . . . . . . . . . . . . . . . . 40

3.3 Case I: Ideal Fourier DIC . . . . . . . . . . . . . . . . . . . . . . 41

3.4 Case II: Cosine Transmittance . . . . . . . . . . . . . . . . . . . . . . 43

3.5 Case III: Randomly Combined Ramps . . . . . . . . . . . . . . . . . 45

4 Experimental Configuration $\quad 49$

4.1 Hardware Design . . . . . . . . . . . . . . . . 50

4.2 Software Design . . . . . . . . . . . . . . . . . . 54

5 Measurement Results and Analysis $\quad 59$

5.1 System Characterization . . . . . . . . . . . . . 60

5.2 Test Phase Object . . . . . . . . . . . . . . . . 62

5.3 Measurement Results . . . . . . . . . . . . . . . . . . . 63

5.4 Results Analysis . . . . . . . . . . . . . . . . . . . 74 
6 Conclusion

6.1 Summary . . . . . . . . . . . . . . . . . . . . . . . 79

6.2 Future Work . . . . . . . . . . . . . . . . . . . . . . . . . 82

$\begin{array}{ll}\text { A Sinusoidal Amplitude Filtering } & 91\end{array}$

B Analytical Analysis of Shearing with Randomly Multiplexed Phase Ramp Filter Functions in a 4-f System

C MATLAB Scripts for the Numerical Simulations

C.1 Ideal DIC . . . . . . . . . . . . . . . . . . . . . . . . . . . . 99

C.2 Cosine Transmittance . . . . . . . . . . . . . . . . . . . . . 104

C.3 Randomly Combined Ramps . . . . . . . . . . . . . . . . . . . 111

C.4 Random Binary Mask Generator Function . . . . . . . . . . . . 119 


\section{List of Tables}

3.1 SLM Functions of the Simulation Cases . . . . . . . . . . . . . . . 39

5.1 Calculated Shear Distance for Number of Cycles in the Blazed Pattern 64 


\section{List of Figures}

1.1 Mechanisms of interaction of light with optically thin transparent objects (phase objects): (a) phase retardation due to optical path length variation across the object, (b) refraction due to refractive index mismatch. . . . . . . . . . . . . . . . .

2.1 (a) Ideal plane wave propagating along the z-axis. (b) Distorted plane wave. (c) Amplitude vs. z-axis of two beams of an ideal plane wave. (d) Amplitude vs. z-axis of two beams of a distorted wavefront. . .

2.2 The effect of a dielectric object on a plane wave impinging upon it. $E_{\text {out }}$ carries information about the amplitude and phase properties of the object. . . . . . . . . . . . . . . .

2.3 Sheared $E$-fields propagating along the z-axis. . . . . . . . . . . .

2.4 4-f system with with cascaded lenses of equal focal lengths. . . . . . .

2.5 Cosine transmittance function. . . . . . . . . . . . . . . . . .

2.6 Two-dimensional phase ramp on the frequency plane with its center at the origin of the coordinate system. . . . . . . . . . . 
2.7 Wrapped phase patterns (also known as blazed patterns) extending the maximum phase retardation for a given SLM dimension, $L$. The blazed pattern number of cycles for these patterns are (a) $T=1$, (b) $T=1.25,(\mathrm{c}) T=2$, and $(\mathrm{d}) T=4 \ldots \ldots \ldots$

2.8 Two (blue and red) blazed phase patterns of the same phase slope sign used for steering the sheared wavefront away from the specular reflection component. The first order diffraction component of the blazed pattern with the larger slope (blue) . . . . . . . . . . .

2.9 Two phase planes with opposite slopes along the x-axis. . . . . . . . .

2.10 Random pattern combination algorithm. . . . . . . . . . . .

2.11 A zoomed-in section of the phase ramps of Figure 2.9 combined by random selection process. . . . . . . . . . . . . . . .

2.12 Lateral phase shifting of a cosine pattern. . . . . . . . . . . . .

3.1 Block diagram of the system simulating the 4 -f system. . . . . . . .

3.2 (a) Two-dimensional plot of the phase function of the object with $R=$ $1 / 4$ and $\kappa=1 / 6$. (b) Cross section plots of the phase function of the object with with $R=1 / 4$ and $\kappa=1 / 3$ (black), $1 / 4$ (red), and 1/6 (blue). 40

3.3 Intensity images from the four-step PSI with amplitude cosine SLM function. . . . . . . . . . . . . . . . . . 41 
3.4 (a) $\Delta \Phi$ from the ideal Fourier DIC case calculated from the four intensity images of Figure 3.3. (b) Cross section view of $\Delta \Phi$ (blue) and direct phase function derivative over 2 pixels (red). . . . . . . . . .

3.5 Cross section view of $\Delta \Phi$ with different shear amounts. . . . . . . 43

3.6 Intensity images from the eight-step PSI with cosine transmittance SLM function. . . . . . . . . . . . . . . . . . . .

3.7 (a) $\Delta \Phi$ with cosine transmittance SLM function calculated from the four-step PSI. (b) Cross section view of $\Delta \Phi$ (blue) and direct phase function derivative over 2 pixels $($ red $) . \ldots \ldots \ldots \ldots$

3.8 (a) $\Delta \Phi$ with cosine transmittance SLM function calculated from the eight-step PSI images of Figure 3.6. (b) Cross section view of $\Delta \Phi$ (blue) and direct phase function derivative over 2 pixels (red). . . . .

3.9 (a) $\Delta \Phi$ with randomly combined ramps SLM function calculated from the eight-step PSI images of Figure 3.10. (b) Cross section view of $\Delta \Phi$ (blue) and direct phase function derivative over 2 pixels (red). . . . 47

3.10 Intensity images from the eight-step PSI with randomly combined ramps SLM function. . . . . . . . . . . . . . . . . . 48

4.1 Schematic of the experimental configuration. . . . . . . . . . 52

4.2 Relative locations of the front and back focal planes of the objective lenses. . . . . . . . . . . . . . . . . . .

4.3 Program algorithm. . . . . . . . . . . . . . . . . 56 
4.4 The illumination path of the experimental configuration. . . . . . . . 57

4.5 The imaging portion of the experimental configuration. . . . . . . . 57

4.6 Close-up view of the object plane of the experimental configuration. . 58

5.1 Measurement of the number of displacement pixels as a function of the number of ramps in a single blazed pattern. Calculated line equation for the linear fit: $y=4.1 x-1.6 \ldots \ldots \ldots \ldots$

5.2 Grayscale bright-field images of the immersion oil $(n=1.515)$ droplet used as test phase object. . . . . . . . . . . . . . . .

5.3 Measurement results of the test droplet with shear direction along the horizontal-axis and $T=0.3\left(S_{\text {image }}=10.824 \mu \mathrm{m}, S_{\text {object }}=0.660 \mu \mathrm{m}.\right)$.

5.4 Measurement results of the test droplet with shear direction along the vertical-axis. $T=0.3\left(S_{\text {image }}=10.824 \mu m, S_{\text {object }}=0.660 \mu m.\right) \quad \ldots$

5.5 Measurement results of the test droplet with shear direction along the horizontal-axis. $T=0.5\left(S_{\text {image }}=18.040 \mu m, S_{\text {object }}=1.100 \mu m.\right)$. .

5.6 Measurement results of the test droplet with shear direction along the vertical-axis. $T=0.5\left(S_{\text {image }}=18.040 \mu m, S_{\text {object }}=1.100 \mu m.\right)$. . . .

5.7 Measurement results of the test droplet with shear direction along the horizontal-axis. $T=1\left(S_{\text {image }}=36.080 \mu m, S_{\text {object }}=2.200 \mu m\right.$. $)$. . . .

5.8 Measurement results of the test droplet with shear direction along the vertical-axis. $T=1\left(S_{\text {image }}=36.080 \mu m, S_{\text {object }}=2.200 \mu m\right.$. $) \quad$. . . 
5.9 Measurement results of the test droplet with shear direction along the horizontal-axis. $T=1.45\left(S_{\text {image }}=52.316 \mu m, S_{\text {object }}=3.190 \mu m.\right) \quad$.

5.10 Measurement results of the test droplet with shear direction along the vertical-axis. $T=1.45\left(S_{\text {image }}=52.316 \mu m, S_{\text {object }}=3.190 \mu m.\right) \ldots$

5.11 Cross section views of $\Delta \Phi$ with different shear amounts. $\ldots \ldots$. . 73 


\section{Glossary}

CCD Charge-Coupled Device.

CRCP Complement of Randomly Combined Pattern.

DIC Differential Interference Contrast.

LUT Look-Up Table.

PSI Phase Shifting Interferometry.

RCP Randomly Combined Pattern.

SLM Spatial Light Modulator. 


\section{Chapter 1}

\section{Introduction}

With the recent advancements of liquid crystal and solid-state technologies, a number of dense, high resolution spatial light modulators (SLM) have emerged. SLM's are devices with two-dimensional arrays of pixels, each pixel with a footprint of only a few $\mu m^{2}$. The pixels of the array can individually be programmed by a computer to manipulate the amplitude or phase of the light incident on them. This has paved the way for precise and unconventional ways to control optical wavefronts without mechanical motion that were impossible before. SLM's have found applications in holography, optical tweezers, spatial filtering, wavefront correction, real-time adaptive optics, microscopy, beam shaping, and more.The focus of this work is on the application of SLM's in phase microscopy and, in particular, Differential Interference Contrast (DIC) microscopy.

This chapter is organized to first present a short historical background and a brief introduction of phase contrast microscopy followed by explanation of the fundamental

physics of DIC microscopy and closing with a literature survey of recent SLM-based phase microscopy techniques. 


\subsection{Phase Microscopy}

The phase of electromagnetic waves is not a directly observable property and it is lost in the intensity detection process due to time averaging. This is the case for our eyes and all electromagnetic detectors. If the interaction of light with an object does not result in detectable intensity variation, the object is said to be transparent and it is, therefore, not observable. However, if a transparent object contains a variation in physical shape or its dielectric constant (index of refraction) it does affect the phase of light incident on it that goes undetected. The goal of phase imaging is to translate this phase interaction to intensity variation that can be detected and observed. Applications of phase microscopy range from imaging and quantitative analysis of biological specimens to metallography, crystalography, and semiconductor failure analysis [1] [2] [3].

From a macroscopic point of view, the interaction of light with transparent objects that affect the phase of the light has two mechanisms: phase retardation due to difference in the optical path length and refraction (also referred to as scattering in some literature). Figure 1.1(a), shows the interaction of a monochromatic, phase coherent plane wave illumination with a homogeneous phase object with thickness comparable to the wavelength of incident light, $\lambda$, and refractive index of $n_{1} \neq n_{0}$. Depending on the shape profile of the object, the incident fields undergo different phase lags across the object while their magnitudes remain unaffected; therefore, the emerging fields are no longer phase coherent but rather contain a variation pattern related to the shape of the object. Due to the time-averaging nature of the square-law detection process that gives the intensity (power) of the fields, the phase information is lost yielding only the square of the magnitude of the fields. The refraction mechanism

is depicted in Figure 1.1(b). Ray optics approach provides a more intuitive prospective 


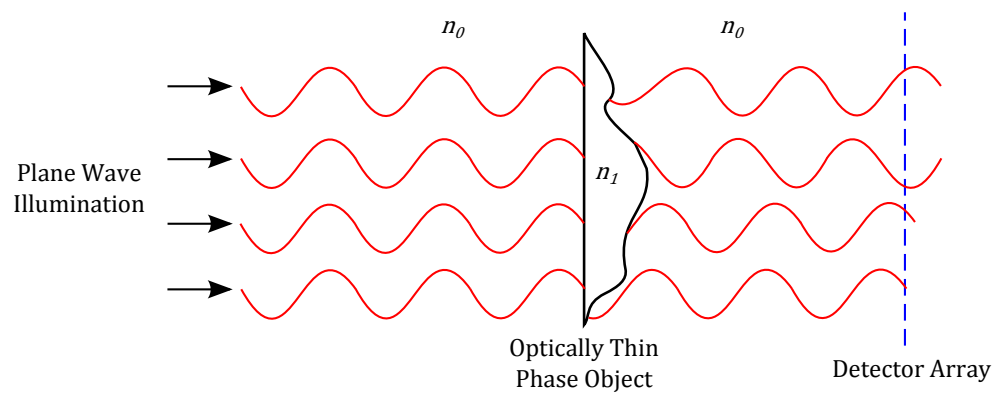

(a)

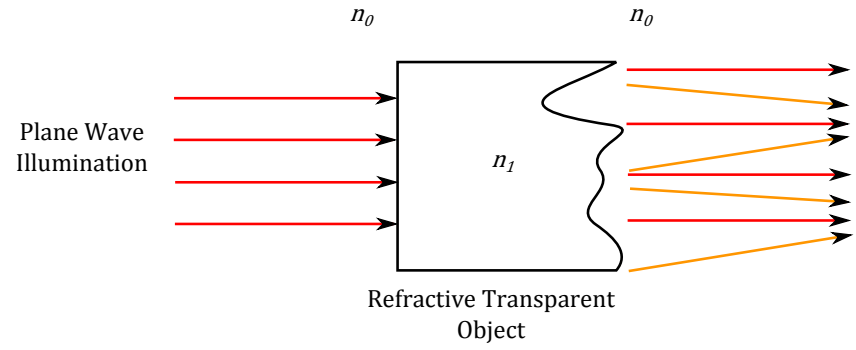

(b)

Figure 1.1: Mechanisms of interaction of light with optically thin transparent objects (phase objects): (a) phase retardation due to optical path length variation across the object, (b) refraction due to refractive index mismatch.

in this case, where the plane wave illumination with parallel rays emerges from the object with some rays in different directions governed by Snell's law of refraction. It should be noted that for optically thin objects with $n_{1} \approx n_{0}$, the majority of the rays passing through remain parallel, and only a small portion of the light is refracted. From the wave optics point of view, this change in direction is equivalent to a shift in the spatial frequency of the fields. These refracted rays carry information about the shape profile of the phase object but the majority of the detected intensity still lies within the unrefracted beams, which gives a very small and difficult-to-observe contrast of the detected intensity.

Two commonly used and commercially available phase microscopy techniques are the Zernike Phase Contrast and the Nomarski DIC imaging [4] [5] that are suitable for qualitative imaging of phase objects. In phase contrast method, the unscattered 
light passing through the object, which for a phase object accounts for the majority of the intensity captured by the detector, is blocked and only refracted light due to difference in the optical path length across the object is allowed to reach the observation (detector) plane. Another common method to increase the contrast between the refracted and unrefracted light is to apply a quarter wavelength relative phase shift between these components with a phase plate. In the resulting intensity image, the brightness level is proportional to the corresponding optical path length at the object plane [6] [7]. The DIC method employs a completely different technique to transduce phase information to intensity. At the core of a DIC microscope are two Nomarksi prisms placed conjugate to each other with the object to be observed in the middle. A Nomarski prism splits an incident beam into two slightly shifted (sheared) beams with orthogonal polarization with respect to each other. These sheared beams propagate through the object and are recombined into path with the second Nomarski prism. Lastly, an analyzer cross interferes these two beams to create an interference pattern. Since each beam propagates along a different path in the object, each one carries amplitude and phase information about the object's effect on the fields along their respective propagation paths. The interference of the sheared beams produces the difference between the effect of the object on the light along those paths converted to intensity. The DIC technique is a common-path interferometry method as no external reference beam is required to generate the interferograms (intensity images). A DIC image provides the gradient of the object's topography along the direction of the shear. Reference [8] provides a comparison of the DIC and phase contrast microscopy techniques along with an interactive online tool that gives a qualitative experience for each of the methods.

While both the DIC and phase contrast microscopy techniques have given a view of the microscopic world that was previously unseen and beyond reach, they do have 
significant shortcomings when it comes to quantitative analysis. In the case of the classic phase contrast method, discrimination of refracted and unrefracted light is done by a phase shift ring where the phase of the background light is shifted by $90^{\circ}$ with respect to the diffracted light. In some cases this discrimination method fails to adequately separate these two components resulting in an artifact in the image called the halo effect. Alternative methods using modern optics techniques such as digital holography [9] [10], structured birefringent pupil filtering [11], and fiber-based differential phase-contrast optical coherence microscopy [12] have been developed providing a more suitable approach for quantitative phase contrast based measurement. In the case of DIC technique there are numerous obstacles for precise, quantitative measurements. The main one is that most commercially available DIC microscopes do not have published specifications for the amount of shear of the system which is needed to calculate the phase gradient from the intensity images. This can be resolved by performing a calibration measurement on a known object [13]. To recover the phase gradient map, phase shifting interferometry is needed which requires manual introduction of a set of at least three phase steps with either known absolute values or equal incremental difference between each step. It should be noted again that the DIC images only provide phase gradient information along the direction of shear. In order to measure the full two-dimensional phase gradient, the object needs to be rotated to be aligned with the shear direction of the microscope which is not very convenient for measuring microscopic objects with great precision.

SLM-based phase microscopy techniques aim to improve upon these traditional techniques by integrating them into automated digital systems to make them more repeatable and flexible and extend their functionalities by means of numerical postprocessing. In the following section, a number of recent publications that have reported use of SLM's in phase microscopy applications with qualitative and quantita- 
tive measurement results are reviewed.

\subsection{Survey of Recent Literature}

Over the past decade, a multitude of reports regarding the use of SLM's for a variety of applications have been published. Many papers in the field of optical tweezers present SLM's as a commonly used component for micro-manipulation of the laser beam as well as aberration correction [14-16]. The use of SLM's as an adaptive optical element for wavefront measurement and correction are reported in a diverse range of configurations and disciplines [17-20]. In this section, a select number of recent peer-reviewed publications concerning the use of SLM in phase microscopy applications are chosen and a brief summary for each is presented. Some of these works have served as prime references in the initial phase of this research work and they have inspired the simulation and experimental methods adapted and presented in the subsequent chapters.

\subsection{Differential Interference Contrast Microscopy with SLM}

In [21], Falldorf et al. used the birefringent property of nematic cells of the SLM on a $45^{\circ}$ polarized incident wavefront to create two slightly spatially apart copies of the wavefront and then recombined them with an analyzer which resulted in the DIC image of the fields. The main principle of this technique is the fact that the refractive index of the slow axis can be locally changed according to the electrically addressed value of the corresponding pixel, whereas the fast axis remains unchanged. A $45^{\circ}$ polarized wave incident on the SLM can be represented by its two orthogonal components: one aligned with the slow axis and the other aligned with the fast axis of the SLM. Therefore, by generating a blazed grating pattern on the SLM one 
component is diffracted into the 1st order (and other higher order but with smaller amplitudes) by the grating while the other component is just reflected. The superposition of these two wave fields by an analyzer is the sheared interference product. The amount and direction of shear can be electronically controlled by changing the period and the orientation of the blazed pattern. In two other publications, Falldorf et al. applied this technique in a normal imaging configuration [22] as in [21] and in the the frequency plane of a 4 -f system [23]. By varying the global phase of the grating pattern, they were able to utilize the four-step phase-shifting interferometry to extract both the amplitude and phase of a monochromatic wavefront generated by deformed water surface and a diffuser.

By building a microscope with LED-based incoherent Köhler illumination and placing a phase-only SLM at the frequency plane of the imaging system in [24], Werber et al. experimented with different phase-only filter patterns to implement the Zernike, DIC, and spiral phase microscopy techniques. Qualitative results from measurements on an injection-molded computer generated hologram and an unstained section of a coney tongue as specimen were reported. Starting with bright-field mode, instead of writing a uniform no-phase modulation on the SLM, a blazed grating was used instead to introduce a carrier frequency to "prevent the superposition of unwanted (created by the SLM) and wanted diffraction orders". Therefore, at the detector plane the bright-field images were laterally shifted away from the axis normal to the SLM plane. This carrier frequency was kept as the default pattern on all the patterns in the experiments with other modes that followed. To realize the dark-field mode by means of phase-only modulation, a small circular area at the center of the SLM where the zero spatial frequencies overlapped was selected with an arbitrary but variable diameter. The phase of the grating in this area was shifted compared to the rest of the pattern which had the effect of shifting the unscattered light to interfere with the scattered 
light. The DIC implementations included patterns having a combination of two blazed gratings with different phase shifts or frequencies with respect to each other. In what was termed W-DIC, half of the SLM's plane contained one grating and the other half contained a slightly shifted version of the same grating pattern. Images obtained in this mode did demonstrate phase gradient profile in the direction of shear but with very poor fine phase gradient details. Another version termed W-DIC-Z extended the pattern of one of the halves to the other half in W-DIC to cover the zeroth order frequencies in an attempt to combine DIC and Zernike methods. This method showed improvements in the finer phase gradients over the W-DIC. Also a full aperture DIC where the pattern is a superposition of two gratings with slightly different frequencies was experimented with but resulted in unclear and unexpected images. Lastly, a modification of the off-axis spiral phase pattern with a constant phase at the center of the pattern was demonstrated to result in anisotropic edge enhancement with directions that are controlled by the phase value of the central constant phase region. All experiments in [24] qualitatively demonstrated the flexibility and power that an SLM can offer in the frequency plane of a microscope by employing different phase microscopy techniques.

Zhao et al. demonstrated shearing and phase shifting interferometry on a macroscopic level in [25] by using a reflectance mode phase-only SLM in the path of an imaging system. In their configuration, an expanded He-Ne laser beam illuminated a $25^{\circ}$ tilted metal plate, then the reflection from this surface was polarized, split, and directed with normal angle to the SLM with an optimized binary phase pattern that generated two diffraction orders of +1 and -1 at $38 \%$ efficiency. This optimization suppressed the zeroth and other higher orders to below $5 \%$ efficiency. The beam splitter placed in front of the SLM directed these diffracted orders to a CCD connected to a computer to capture the intensity interference pattern. To recover the 
phase distribution from the object (reflection from the metal plate), four-step phase shifting interferometry was done by laterally shifting the SLM's pattern by an appropriate number of pixels. The displacement distance between the two diffracted orders was $3 \mathrm{~mm}$ and the recovered unwrapped phase distribution had the profile of a semi-linear ramp with a slope of 1.25 radians $/ \mathrm{mm}$. Although not a microscopy experiment, this work provides a very similar overall structure to phase microscopy imaging with SLM's. Also of note in this work is the technique of using an optimized binary phase grating pattern on the SLM in reflectance mode without a tilt angle and suppressing of the undesired specular reflection from the SLM. Other similar works have reported different techniques to minimize the zeroth diffraction order component and it is one of the important practical factors to be carefully considered in using SLM's for differential contrast interferometry.

A modified Michaelson interferometry technique was developed in [26] where the magnified light from the specimen is split into two paths: one with a reflectance mode phase-only SLM placed at the termination of the arm to provide a copy of the specimen's image and another one with an objective lens facing a mirror to create an inverted copy of the specimen's image. The reflected fields from these two paths are combined and interfered at the CCD plane. Then the SLM is used to apply a series of uniform phase shifts along the propagation path to allow phase shifting interferometry to extract the phase information from the resulting intensity fringe patterns. Test objects for their experimental results included polystyrene beads and red blood cells. Unlike the aforementioned works where the SLM was placed in the frequency plane of the imaging system, phase shifting interferometry was done in the traditional way in this work instead of creating an SLM pattern and laterally shifting it. 


\subsection{Spiral Phase Microscopy}

Another phase imaging technique that has been the subject of extensive recent research and investigation is spiral phase filtering. This in effect creates an interference of the original image of the object with a two-dimensional Hilbert transform of itself [27]. The one dimensional Hilbert transform of a function $f(x)$ with Fourier transform of $F(\omega)$ is done by applying a $-\pi / 2$ phase shift to the negative frequency components of $F(\omega)$ and a $+\pi / 2$ phase shift to the positive frequency components of $F(\omega)$. Performing this transform on the image of an object creates an edge enhanced image in one direction only. For isotropic edge enhancement a two-dimensional transform is required. In the two-dimensional frequency plane, this requires that the one-dimensional Hilbert transform be performed on each radial of the frequency plane thus yields a spiral phase filter function. The theoretical development of the spiral phase function (also known as radial Hilbert transform, phase rotor, and vortex filter) is presented in detail in [28] and [27]. Davis et al. also presented qualitative measurements of 1D, orthogonal, and radial Hilbert transform on a circular aperture as the object in [27] by using a transmissive phase-only SLM in the frequency plane of an imaging system and showed that an isotropic edge enhancement is possible by means of spiral phase filtering.

In a 4-f imaging system, applying the spiral phase function in the frequency plane is possible in two ways: 1) direct phase function or 2) holographic reconstruction by placing the hologram of the interference of a spiral phase modulated wavefront with a reference wave. The hologram in the latter method is created numerically and it is usually based on an off-axis reference illumination whose interference with a spiral phase modulated wavefront creates a fork-like fringe pattern [29]. Both amplitude and phase holograms can be used where the amplitude hologram is encoded by binary 
(black/white) values and the phase hologram encoded in gray scale and also has a blazed grating pattern superposed on the fork-like pattern. By illuminating this numerically generated hologram with the reference wave (in this case the zerothorder components in the frequency plane) the spiral wavefront is reproduced in the first diffraction order of the hologram [30]. The advantage of creating the phase hologram on a phase-only SLM is that it provides the flexibility to digitally adjust the direction of the diffracted beam and its diffraction efficiency.

Although, Davis et al. demonstrated the first SLM-based spiral phase filtering on a macroscopic amplitude object in [27], the first successful qualitative phase object microscopy by means of spiral phase filtering was demonstrated by Fürhapter [31]. Through a series of publications that followed shortly afterwards [32-38] they explored the properties of the images obtained through spiral phase filtering and also further advanced their technique for quantitative measurements of phase objects.

In [31], Fürhapter et al. provided numerical simulations comparing Zernike phase contrast to filtering with spiral phase filter on a weak phase object $(0.25 \%$ of the wavelength) and showed improved contrast from $6 \%$ for the phase contrast to $100 \%$ for the spiral phase filtering. The reason for such drastic improvement is that the background of the phase contrast technique is modulated by the phase object while it remains zero for the spiral phase method except at the edges of the object. The experiments used a modified microscope illuminated with a 780nm laser diode and a phase-only SLM in the frequency plane of their extended 4-f imaging system. Using the same configuration, they demonstrated bright-field, dark-field, phase contrast, and spiral phase microscopy. For the bright-field, a blazed grating pattern was written on the SLM to diffract the light into the first diffraction order and onto a CCD. A circular region in the center of the SLM's blazed pattern was left blank to block 
the lowest spatial frequencies to create the dark-field images. That same circular region was replaced by a laterally shifted blazed pattern to steer the zeroth-order beam to interfere with the higher spatial frequency components (scattered light from the object) which created the phase contrast images. And lastly, they programmed the SLM with a digital phase hologram to reconstruct the spiral phase mode that gets multiplied by the Fourier transform of the object's field. Their phase object included a water coated scratched glass and a thin layer of water and oil mixture. Compared to the bright-field images, the spiral phase technique did show isotropic contrast at objects' phase steps. Although the experiments remained at qualitative level, the authors concluded that imaging of phase objects as optically thin as $1 \%$ of the wavelength is possible with spiral phase contrast microscopy.

An important property of the spiral phase filter function that gives rise to the isotropic edge enhancement is its singularity at its center. Where the spiral phase is used as a filter mask in the frequency plane, the low spatial frequencies would vanish and only the higher order components would undergo phase modulation. This is done by superimposing a blazed pattern on the SLM and diffracting all spatial frequency orders to the detector plane but the low spatial frequencies. On the other hand, the consequence of violating the central singularity and angular symmetry of the spiral phase function is the loss of isotropy of the image resulting in a topographic image with relief-like shadow effect [33]. This effect arises because the low spatial frequency components would also be diffracted with a defined relative phase with respect to other higher orders and would transform into a plane wave at the detector plane and interfere with the Hilbert transform of the object. Separately controlling the phase of the blazed pattern in the central area of the hologram changes the incident angle of this plane wave and causes the direction of the shadow effect to rotate. The same shadow effect can be achieved by angular rotation of the hologram pattern around 
the central blazed pattern. By stepping the phase of this area from 0 to $2 \pi$ with 12 equal increments and summing the resulting images for each step, Jesacher et al. in [33] demonstrated isotropic edge amplification of the image of a human cheek cell. By applying numerical inverse Hilbert transform to the summed images, they obtained a phase contrast image of the cell with an improved phase gradient resolving power over the Nomarski DIC technique. The diameter of the central area of the SLM's pattern was estimated from the diameter of the sharply imaged illumination fiber output. The diameter was varied experimentally until maximum shadow contrast was achieved.

In [35], Brenet et al. developed the theoretical basis for quantitative magnitude and phase measurement of complex samples using the same phase stepping and integration technique and also presented experimental measurements and post-processed results for a Richardson microscope test slide and human cheek cell. The experiments in this work were designed for white light illumination. While still showing qualitative agreement with the object's profile, the results for the Richardson pattern showed nearly $40 \%$ error in phase measurement compared to the images obtained by an atomic force microscope. The cited reason for this large error was attributed to the "limited spatial coherence of the white-light illumination". This was verified when the illumination source was replaced by a coherent laser diode and a $30 \%$ accuracy improvement was achieved. However, the longitudinal coherence of the laser also created artifacts due to laser speckle. Contrary to the initial speculations, it was concluded that with an additional calibration step the spiral phase method can still be used for quantitative measurement of complex samples.

In [37], Maurer et al. revisited the assumption made about the reference plane wave and the way it was redirected in the frequency plane. In the earlier publications 
where a large error was present, it was assumed that the reference wavefront had a uniform amplitude distribution. However, when an object is present in the object plane of the microscope, low frequency Fourier transform components broaden as a consequence of diffraction. With this, the diameter of the central disk on the SLM pattern needs to expand to adapt to the larger point spread function. Maurer experimentally adjusted the diameter of the central disk and made a series of measurements on polystyrene beads in immersion oil with known index of refraction. Since the properties of the test objects were known, he was able to vary the diameter of the central disk and determine the amount that gave the smallest measurement error. Also a calibration step was added in the process that measured the amplitude distribution of the reference wave and applied it in the post processing calculations. With these modifications to the system, the measurement accuracy was $99.4 \%$.

\subsection{A Look Ahead}

The literature review in the previous section provides the state of the art in modern phase microscopy. It indicates what the required elements and methodologies exist for a DIC microscope with quantitative measurement capability. The following is a summary of some of the important lessons learned from surveying these literature that have been used in defining this research project and the design of the experiments.

The use of both common-path and double-path interferometry techniques with SLM's have been reported. The common-path method lends itself to a more compact realization that is more suitable for a microscope system. For accurate quantitative measurements, both the Zernike and spiral phase contrast approaches with an SLM face the challenge of knowing the point spread function of the system for every object under test. This is because an area in the center of the pattern on the SLM, that 
is placed in the frequency plane of the imaging system, needs to be manipulated to control the low spatial frequencies. Knowing the optimal area requires an extensive calibration step. Shearing interferometry does not have this problem but instead the specular reflection from the SLM needs to be considered. Configurations containing both off-axis and on-axis arrangement for the SLM are possible. Furthermore, the reflection from the SLM can be incorporated as one of the mechanisms in the shearing operation. Therefore, shearing interferometry and specifically the DIC technique was selected for this work.

Phase shifting interferometry (PSI) is necessary for the extraction of the phase information from interferograms. Several papers in the reviewed literature reported placement of the SLM in the frequency plane of a 4-f system and performed PSI by lateral shifting of the pattern on the SLM. This is a convenient and compact way to implement PSI.

The measurement process in modern microscopes is performed by a complex closed-loop control system that includes development of a custom software and, in most cases, some custom hardware. The building of such microscope system requires a systematic and methodical approach to the design of the system to ensure robustness. Also for the validation of both qualitative and quantitative measurements, a numerical simulation program is a helpful tool to compare the measurements with the expected results.

A common construction of a weak phase object used for quantitative measurements is immersed polystyrene beads in immersion oil. Polystyrene beads with precisely known physical shape and index of refraction are commercially available. Placing them in immersion oil with the index of refraction very close to that of the beads', creates a small but known optical path length difference. The expected measurement 
results from that path length difference can be calculated as a comparison reference. A few papers also reported comparison of their optical microscopy measurements of an arbitrary object with the measurements obtained from an electron microscope. Two important metrics used to characterize a DIC microscope are 1) the amount of shear and 2) the smallest resolvable phase gradient that can be measured. The latter is typically a small percentage of the wavelength of the illumination source. Therefore, knowledge of the phase object to sub-wavelength orders is necessary for quantitative characterization.

Among the publications that were reviewed, the work in [39] followed by [40] can without a doubt be ranked among the most novel and creative approaches in the use of SLM in a microscope systems. The SLM used in these works is placed in the frequency plane of a custom-built microscope configuration and acts as spatial filter mask. The pattern for the filter masks are two blazed patterns with slightly different frequencies that are randomly interleaved together on the SLM. This forms a diffractive surface in the frequency plane with two closely spaced and adjustable diffraction orders that creates an interference of two spatially shifted images of the object fields at the detector plane. In [40], quantitative measurement results made on polystyrene beads immersed in index matching oil with index of refraction difference $\Delta n=0.06$ with error as small as $3 \%$ were reported. The cited paper [41] for the motivation to randomly combine the two patterns addresses the need for such method for multiplexing multiple functions on a phase-only SLM. Although still applicable to the DIC operation, this cited reference covers a broader and more generic base to justify why pattern randomization for such filter masks is needed.

It was concluded that an alternative point of view that is more suitable in the context of the DIC technique can be presented. In deriving the required SLM function 
from Fourier optics analysis of the shearing operation, an amplitude-only function for the SLM is obtained and the purpose of pattern interleaving of phase-only functions is to replicate the effect of an amplitude-only SLM. This work presents an alternative analysis to explain the need for random combination of the two blazed patterns. Additionally, a novel PSI method that enables the use of amplitude-only SLM for DIC microscopy is developed. This PSI method can also be used to remove the effects of the specular refection when a reflectance mode phase-only SLM is used as the filter mask in the DIC system.

The subsequent chapters are arranged as follows. In Chapter 2, the theoretical Fourier optics analysis of a DIC system with a 4 -f system is presented. Chapter 3 includes numerical simulation results developed to validate the theoretical predictions of Chapter 2 as well as to provide a reference for validation of the measurement results. Chapter 4 contains details on the laboratory implementation of an experimental configuration inspired by the work of [40] using a phase-only SLM and the random pattern combination. The measurement results and discussion are presented in Chapter 5. Lastly, Chapter 6 contains a conclusion and a discussion of future work. 


\section{Chapter 2}

\section{Fourier DIC Microscopy: Theory}

Linear optical signal processing systems may be achieved by exploiting the Fourier transforming property of lenses and a filter mask placed as a spatial light modulator (SLM) in the Fourier transform (frequency) plane of a 4-f optical system. Programmable SLM's permit dynamic manipulation of a wavefront using the Fourier transform pairs and linear systems theory. Some examples of novel and unconventional techniques stemming from this are new approaches to classic microscopy techniques such as Zernike and DIC that recently have experimentally been used in a variety of applications. These new techniques improve repeatable quantitative measurements by eliminating the need for manual and mechanical movements. The aim of this chapter is to provide a thorough theoretical and mathematical basis for a new DIC technique implemented in a 4-f system with a phase-only SLM that is primarily based on the works in [39] and [40]. This also lays the foundation for understanding and analyzing the simulations and experimental results of chapters 3 and 5 .

This chapter first gives an overview of wavefront distortion followed by the principles of wavefront shearing that is the underlying mechanism of forming DIC images and phase gradient measurement. Further sections provide details on 4-f systems 
and how shearing can be done by means of the Fourier shift theorem. Lastly, implementation of this technique with a phase-only SLM that requires a deviation from conventional approaches is discussed.

\subsection{Wavefront Distortion}

Electromagnetic waves propagating through any medium with inhomogeneous dielectric and absorption properties undergo phase distortion and amplitude attenuation. This concept is best described by considering the distortion of an ideal plane wave where the fields on the surface of an imaginary plane normal to the propagation vector have the same magnitude and phase. For an ideal plane wave propagating in a vacuum, the field magnitude remains unchanged and phase coherency is preserved at any position along the propagation direction. The effects of the medium on the plane wave are the distortion of the fields' phase coherency and non-uniform attenuation. Figure 2.1(a) shows a plane wave propagating along the z-axis where the dots in the $\mathrm{x}-\mathrm{y}$ plane mark the peak of the electric field waves, for example, as in Figure 2.1(c). Figures 2.1(b) and (d) show a distorted wavefront.

The time-harmonic monochromatic plane wave with wavelength $\lambda$ is given by:

$$
E(\vec{r}, t)=A e^{i(\vec{k} \cdot \vec{r}-\omega t+\Phi)}
$$

where $\vec{k}$ is the wavevector whose magnitude is the wavenumber, $2 \pi / \lambda$, and its direction is the wave's propagation direction, $\omega$ is the angular frequency, and $\Phi$ is the phase. For analyses concerning the amplitude and phase of the wave, the time and position dependent terms can be omitted and added to the final expression without affecting the results. In the case of a wave traveling along the z-axis as in Figure 2.1, this 


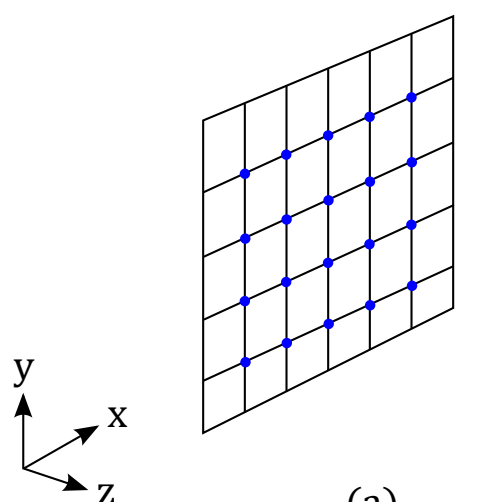

(a)

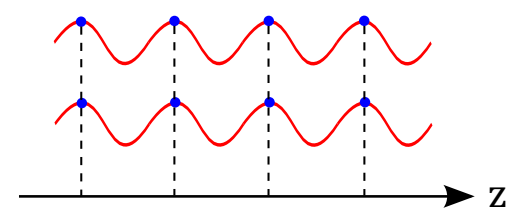

(c)

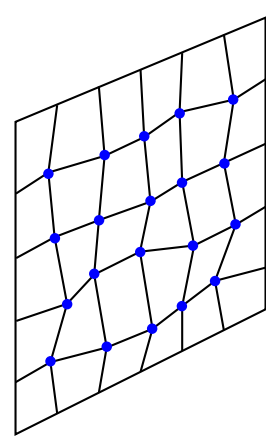

(b)

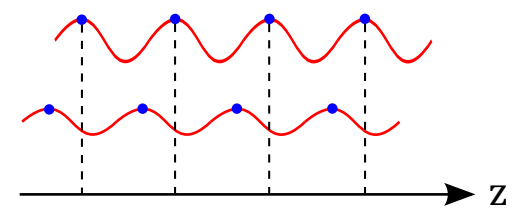

(d)

Figure 2.1: (a) Ideal plane wave propagating along the z-axis. (b) Distorted plane wave. (c) Amplitude vs. z-axis of two beams of an ideal plane wave. (d) Amplitude vs. z-axis of two beams of a distorted wavefront.

yields the two-dimensional phasor expression:

$$
E(x, y)=A e^{i \Phi}
$$

It should be noted that for a plane wave, since both the amplitude and phase are uniform on the $\mathrm{x}-\mathrm{y}$ plane, neither terms on the right hand side of Eq. 2.2 have spatial dependency (Figure 2.1(a)). However, this changes for a distorted wavefront due to unequal phase and amplitude distribution of the fields (Figure 2.1(b)). In the context of microscopy, Figure 2.2 shows the effects of an object on the electric field of an incident monochromatic plane wave illumination where $E_{\text {out }}(x, y)$ is the expression for the electric field as it is exiting the object. $A_{o b j}(x, y)$ and $\Phi_{o b j}(x, y)$ give the amplitude and phase distortion of the output wavefront due to the object respectively. 


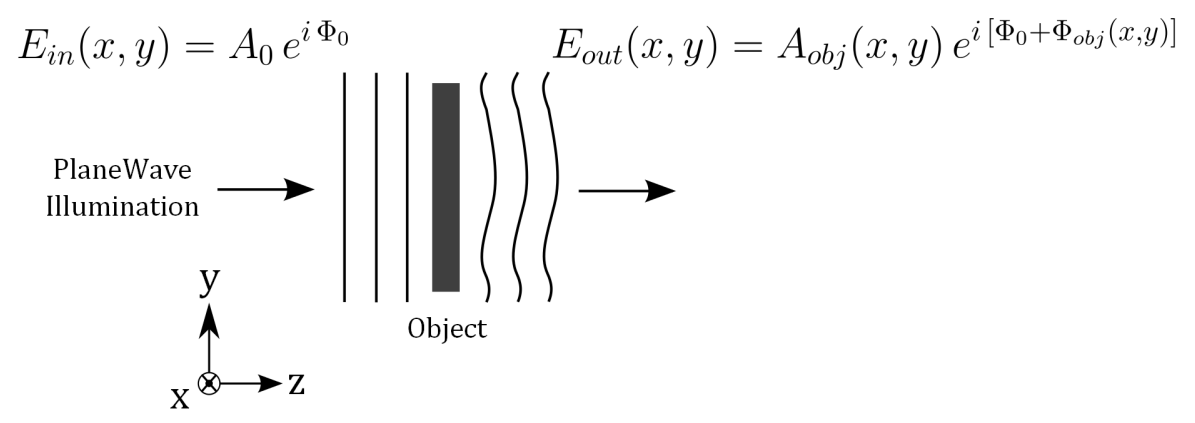

Figure 2.2: The effect of a dielectric object on a plane wave impinging upon it. $E_{\text {out }}$ carries information about the amplitude and phase properties of the object.

$E_{\text {out }}$ now contains information about the amplitude and phase properties of the object. Limiting the application to objects with thickness smaller than the wavelength of the light (also known as optically thin objects), $A_{o b j}$ contains the absorption property as well as diffraction effects, and for an homogeneous object, $\Phi_{o b j}$ contains shape profile of the object given by $h(x, y)=\Phi_{o b j} \lambda / 2 \pi$. In the case of a non-homogeneous object, both the shape profile and dielectric constant (refractive index) variation of the object are embedded in $\Phi_{o b j}$.

The intensity of the light emerging from the object detected by a detector array is given by:

$$
\begin{aligned}
I_{\text {out }}(x, y) & =E_{\text {out }}(x, y) E_{\text {out }}^{*}(x, y) \\
& =A_{o b j}(x, y) A_{o b j}(x, y) e^{i\left[\Phi_{0}+\Phi_{o b j}(x, y)\right]} e^{-i\left[\Phi_{0}+\Phi_{o b j}(x, y)\right]} \\
& =A_{o b j}(x, y)^{2}
\end{aligned}
$$

Where $E_{\text {out }}^{*}(x, y)$ is the complex conjugate of $E_{\text {out }}(x, y)$. In the intensity measurement process the phase information vanishes and only the square of the field magnitude is detected. Recovering the phase term from intensity measurements is the goal of phase microscopy. 
The next section provides the theoretical basis of the DIC technique, where the derivative of the phase term is extracted from a series of intensity measurements.

\subsection{Principles of Differential Interference Contrast}

As the name suggests, DIC uses interference to create an intensity pattern that is related to the wavefront under test. To better understand the DIC technique and its functionality compared to other interferometric measurement methods, a brief review of interferometry is presented. Interferometry is a measurement technique used to translate the phase variation of a wavefront to an intensity pattern by introducing a reference wavefront to interfere with the wavefront under test. The intensity pattern (also known as the interferogram) has a non-linear relationship with the phase difference of the two interfered wavefronts. There are various interferomtry techniques used for a variety of applications ranging from optical and radio astronomy to light and electron microscopy. One of the main differentiating factors in the family of interferometry techniques is the method by which the reference wavefront is introduced: external or self-referencing. An external reference is a known and usually an undistorted wavefront whose phase offset can be adjusted such as the ones in Michaelson and Mach-Zehnder interferometers, where the illumination path is split into two ways. One path propagates through the medium under test and the other path serving as a reference bypasses the medium and is summed and interfered with the wavefront distorted by the medium. In the case of the self-referencing interferometer the waveform under test is interfered with a (typically a delayed or spatially shifted) copy of itself.

The DIC technique is a self-referencing and common-path interferometry technique where the interference takes place between two copies of a wavefront that are 
slightly separated (sheared) from each other in space. The shearing direction is normal to the propagation vector. Figure 2.3 shows an example of sheared $E$-field of a wavefront traveling along the z-axis. Setting the origin of the analysis coordinate about the center of shear, each wavefront can be assigned a displacement vector: $\vec{S}$ and $-\vec{S}$. Continuing with the example in Figure 2.2 with $\Phi_{0}=0$ and omitting the

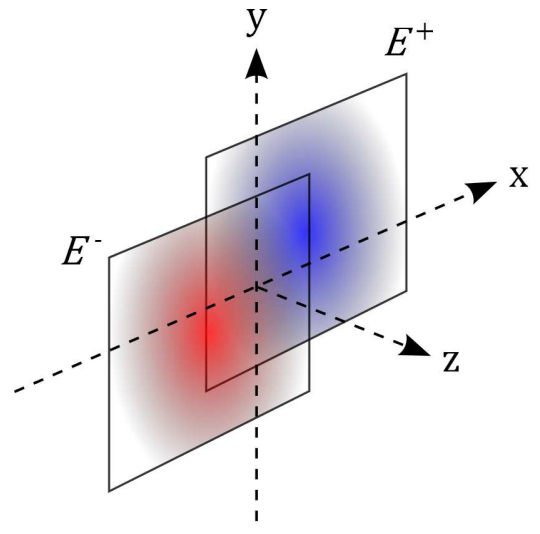

(a)

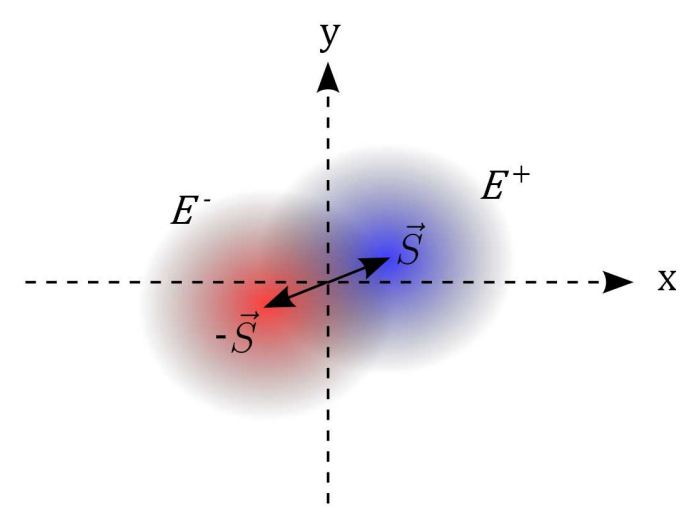

(b)

Figure 2.3: Sheared $E$-fields propagating along the z-axis.

subscript obj for brevity the $E$-field expression for output wavefront becomes:

$$
E_{\text {out }}(\vec{r})=A(\vec{r}) e^{i \Phi(\vec{r})}
$$

And the sheared fields are:

$$
\begin{aligned}
& E^{+}(\vec{r})=\gamma^{+} E_{\text {out }}(\vec{r}+\vec{S}) \\
& E^{-}(\vec{r})=\gamma^{-} E_{\text {out }}(\vec{r}-\vec{S}),
\end{aligned}
$$

where $\vec{r}$ is the two-dimensional coordinate vector on the plane normal to the propagation vector and $\gamma^{+}$and $\gamma^{-}$are the attenuation coefficients due to splitting. For a 
z-axis traveling wave, the above terms become:

$$
\begin{aligned}
E^{+}(x, y) & =\gamma^{+} A\left(x+S_{x}, y+S_{y}\right) e^{i \Phi\left(x+S_{x}, y+S_{y}\right)} \\
& =A^{+} e^{i \Phi^{+}} \\
E^{-}(x, y) & =\gamma^{-} A\left(x-S_{x}, y-S_{y}\right) e^{i \Phi\left(x-S_{x}, y-S_{y}\right)} \\
& =A^{-} e^{i \Phi^{-}}
\end{aligned}
$$

Here the amplitude terms of each of the sheared fields have been lumped together as $A^{+}$and $A^{-}$. The intensity of the interference between the two sheared fields is given by:

$$
\begin{aligned}
I(x, y) & =\left|E^{+}(x, y)+E^{-}(x, y)\right|^{2} \\
& =\left[E^{+}(x, y)+E^{-}(x, y)\right] \times\left[E^{+*}(x, y)+E^{-*}(x, y)\right] \\
& =A^{+}+A^{-}+2 A^{+} A^{-} \cos \left(\Phi^{+}-\Phi^{-}\right) \\
& =A^{+}+A^{-}+2 A^{+} A^{-} \cos (\Delta \Phi)
\end{aligned}
$$

Where $\Delta \Phi \equiv \Phi\left(x+S_{x}, y+S_{y}\right)-\Phi\left(x-S_{x}, y-S_{y}\right)$. Further defining $\alpha \equiv A^{+}+A^{-}$ and $\beta \equiv 2 A^{+} A^{-}$:

$$
I(x, y)=\alpha+\beta \cos (\Delta \Phi)
$$

This equation is the classic intensity fringe pattern resulting from interference of two planar wavefronts. The first term is a constant (background light) and the second term is the cosine term whose argument is the phase difference. Unlike Eq. 2.3, the phase information has not vanished but instead its spatial difference along the shear direction appears in a cosine term. For small shear distances comparable to the resolution of the microscope, the phase gradient along the shear direction is given by 
the following approximation:

$$
\frac{\partial \Phi_{o b j}}{\partial r} \approx \frac{\Delta \Phi}{2|\vec{S}|}
$$

From this result, it can be seen that the shear vector controls two properties of the measured phase gradient: the gradient resolution (i.e. the distance over which the phase difference is measured), given by $2|\vec{S}|$, and the gradient direction, given by $\hat{s}=\vec{S} /|\vec{S}|$.

A further step is required to recover the phase difference term from Eq. 2.8. This can be done using Phase Shifting Interferometry (PSI) where an additional variable phase term is introduced in the cosine argument to create a series of intensity measurements [42]. Including the PSI step, the generalized intensity expression becomes:

$$
I_{j}(x, y)=\alpha+\beta \cos \left(\Delta \Phi+\theta_{j}\right) \quad j=1,2,3, \ldots
$$

In the case of four-step PSI where:

$$
\begin{aligned}
& \theta_{1}=0 \quad \rightarrow \quad I_{1}=\alpha+\beta \cos (\Delta \Phi) \\
& \theta_{2}=\pi / 2 \quad \rightarrow \quad I_{2}=\alpha-\beta \sin (\Delta \Phi) \\
& \theta_{3}=\pi \quad \rightarrow \quad I_{3}=\alpha-\beta \cos (\Delta \Phi) \\
& \theta_{4}=3 \pi / 2 \quad \rightarrow \quad I_{4}=\alpha+\beta \sin (\Delta \Phi)
\end{aligned}
$$

The phase difference expression can be recovered using the following equation:

$$
\Delta \Phi=\tan ^{-1}\left(\frac{I_{4}-I_{2}}{I_{1}-I_{3}}\right)
$$




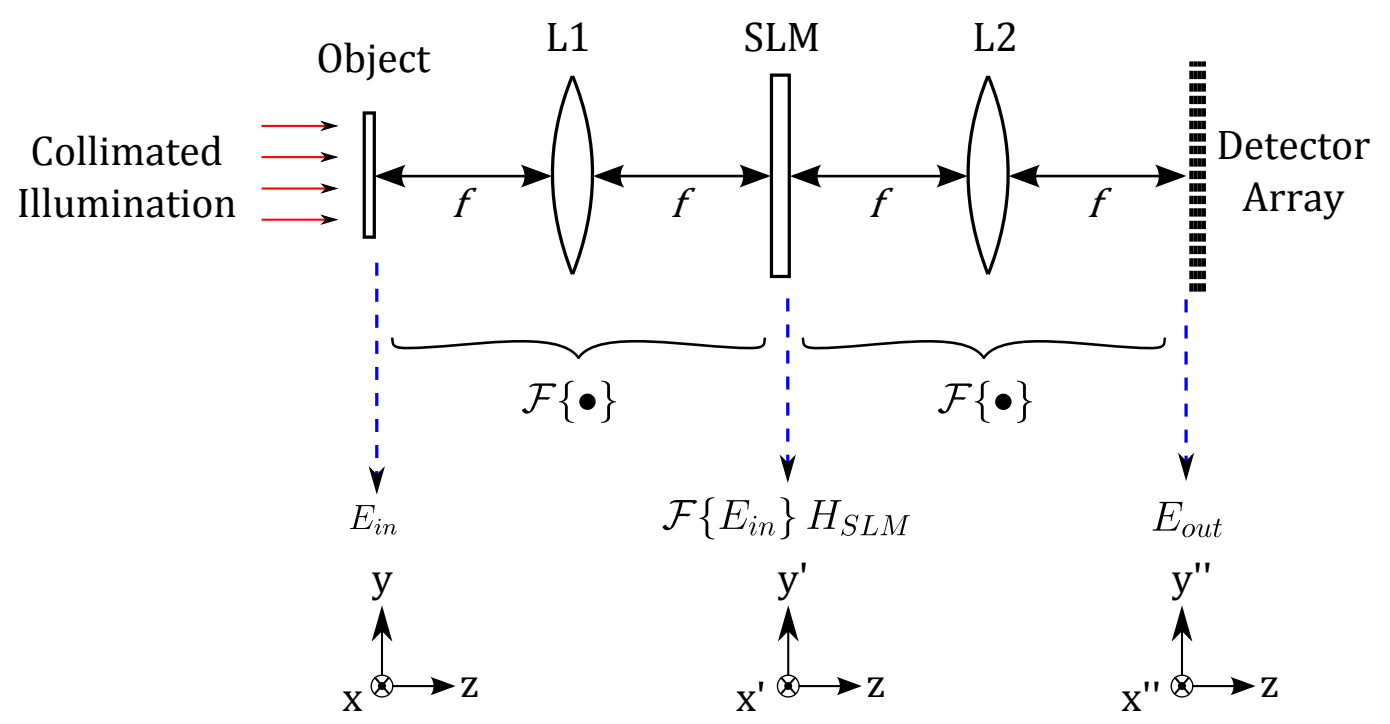

Figure 2.4: 4-f system with with cascaded lenses of equal focal lengths.

\subsection{Wavefront Modulation with a 4-f System}

An optical 4-f system with a filter mask can be used as an analog optical processing system to manipulate a wavefront's amplitude and phase. Figure 2.4 shows a 4-f system where two lenses $L 1$ and $L 2$ with equal focal lengths are separated by the sum of their focal lengths. The input wavefront is placed at the back focal plane of the first lens and by the Fourier transforming property of lenses the input fields are transformed to the spatial frequency domain at the front focal plane of $L 1$. Here a programmable SLM multiplies the transformed fields by a spatial complex function $H_{S L M}\left(f_{x}, f_{y}\right)$. The second lens placed one focal distance from the SLM performs another Fourier transform to convert the modulated fields back to the spatial domain on a detector array. The SLM's function has the following form:

$$
H_{S L M}\left(f_{x}, f_{y}\right)=B\left(f_{x}, f_{y}\right) e^{i \psi\left(f_{x}, f_{y}\right)}
$$

Where $f_{x}=\frac{x^{\prime}}{\lambda \mathrm{f}}, f_{y}=\frac{y^{\prime}}{\lambda \mathrm{f}}, B\left(f_{x}, f_{y}\right)$ and $\psi\left(f_{x}, f_{y}\right)$ are the amplitude and phase functions applied to the Fourier transformed fields by the SLM respectively. 
The output fields of the 4-f system, $E_{\text {out }}$, are the convolution of the SLM's function with the input fields which in the example of Figure 2.4 are the fields exiting the object. This relationship can be used to apply a specific relationship between the output and input fields by programming the appropriate SLM function. Mathematically, there are two ways to express the output of the 4-f system:

$$
\begin{aligned}
& E_{\text {out }}=E_{\text {in }} * \mathcal{F}\left\{H_{S L M}\right\} \\
& E_{\text {out }}=\mathcal{F}\left\{\mathcal{F}\left\{E_{\text {in }}\right\} H_{S L M}\right\}
\end{aligned}
$$

This provides a powerful tool to employ the linear systems theory and Fourier transform pair relationships to manipulate a wavefront.

It should be emphasized that unlike typical linear systems where a Fourier transform is followed by an inverse Fourier transform, the second lens in the 4-f system performs a second consecutive Fourier transform. This is still the same as having an inverse Fourier transform except that the output is mirrored compared to the input due to the following property of Fourier transform:

$$
\mathcal{F}\{\mathcal{F}\{g(x, y)\}\}=g(-x,-y)
$$

Also if the focal lengths of $L 1$ and $L 2$ are not equal, the output fields become scaled by the magnification factor $M=\frac{f_{1}}{f_{2}}$ where $f_{1}$ and $f_{2}$ are the focal lengths of the first and second lens respectively.

Most commercially available programmable SLM's are either amplitude-only or phase-only which means that only one of the parameters of Eq. 2.12 can be controlled. Throughout this work, only the phase-only SLM type is considered. 


\subsection{Wavefront Shearing with a 4-f System}

Thus far the mechanisms of wavefront distortion by an object and a way to recover the phase difference by shearing and PSI have been presented. The next step is to utilize the 4-f system to perform differential contrast interference by wavefront shearing and PSI. Here the task is to find an expression for $H_{S L M}$ that will convert the input wavefront:

$$
E_{i n}(x, y)=A_{o b j}(x, y) e^{i \Phi_{o b j}(x, y)}
$$

to:

$$
E_{\text {out }}(x, y)=\frac{1}{2} E_{\text {in }}\left(x+S_{x}, y+S_{y}\right)+\frac{1}{2} E_{\text {in }}\left(x-S_{x}, y-S_{y}\right)
$$

that is the sum of two spatially shifted versions of the input fields. The spatial shifting can be realized by using the Fourier shift theorem that states multiplication by a linear phase ramp in one domain translates to shifting of the function in the other domain:

$$
\begin{aligned}
& E_{i n}\left(x+S_{x}, y+S_{y}\right) \longleftrightarrow \mathcal{F}\left\{E_{i n}\right\}\left(f_{x}, f_{y}\right) e^{i 2 \pi\left(S_{x} f_{x}+S_{y} f_{y}\right)} \\
& E_{i n}\left(x-S_{x}, y-S_{y}\right) \longleftrightarrow \mathcal{F}\left\{E_{i n}\right\}\left(f_{x}, f_{y}\right) e^{-i 2 \pi\left(S_{x} f_{x}+S_{y} f_{y}\right)}
\end{aligned}
$$

This yields the following expression for the SLM's function:

$$
\begin{aligned}
H_{S L M}\left(f_{x}, f_{y}\right) & =\frac{1}{2}\left[e^{i 2 \pi\left(S_{x} f_{x}+S_{y} f_{y}\right)}+e^{-i 2 \pi\left(S_{x} f_{x}+S_{y} f_{y}\right)}\right] \\
& =\cos \left[2 \pi\left(S_{x} f_{x}+S_{y} f_{y}\right)\right]
\end{aligned}
$$


Or in terms of Eq. 2.12:

$$
\begin{aligned}
& B\left(f_{x}, f_{y}\right)=\cos \left[2 \pi\left(S_{x} f_{x}+S_{y} f_{y}\right)\right] \\
& \psi\left(f_{x}, f_{y}\right)=0
\end{aligned}
$$

This means that the SLM's function is an amplitude-only cosine function. There is, however, a physical limitation to the implementation of it in this form. The cosine values alternate between the positive and negative values but a negative amplitude for the fields is meaningless since they can only take on values from 0 to their peak level. An offset cosine function can be used instead with the transmittance behavior of Figure 2.5 where the SLM's function would take the following form:

$$
H_{S L M}=\frac{1}{2}+\frac{1}{2} \cos \left[2 \pi\left(S_{x} f_{x}+S_{y} f_{y}\right)\right]
$$

This is a sinusoidal amplitude grating with 3 diffraction orders: $-1,0$, and +1 . The zeroth term adds two additional half shear phase difference terms to the intensity expression of Eq. 2.8 that require yet another phase stepping process to obtain the desired phase difference term for the DIC. Since the objective of this work is to implement the DIC technique with a phase-only SLM, further discussion of amplitudeonly SLM function is deferred to Appendix A.

Starting again with Eq. 2.17, $e^{i 2 \pi\left(S_{x} f_{x}+S_{y} f_{y}\right)}$ and $e^{-i 2 \pi\left(S_{x} f_{x}+S_{y} f_{y}\right)}$ are two tilted phase planes (such as glass wedges) with opposite slopes of orthogonal components $2 \pi S_{x}$ and $2 \pi S_{y}$ in the frequency plane of the 4 -f system. Figure 2.6 shows an example

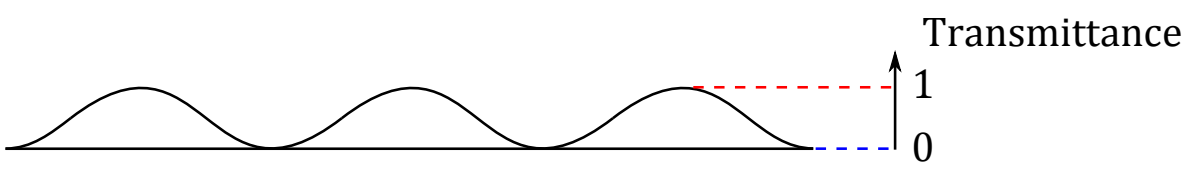

Figure 2.5: Cosine transmittance function. 
of such phase plane with a negative slope along the $f_{x}$ direction. The slope of these phase planes determines the spatial shifting in the detector plane. Considering the example of Figure 2.6, the slope of the tilted plane on a square SLM with the side dimension $L$ is given by:

$$
\begin{aligned}
& 2 \pi S_{x}=\frac{\psi_{x_{\text {peak }}}}{f_{x_{\text {max }}}}=\frac{\psi_{\text {xpeak }}}{L / 2 \lambda \mathrm{f}} \\
& 2 \pi S_{y}=\frac{\psi_{y_{\text {peak }}}}{f_{y_{\text {max }}}}=\frac{\psi_{y_{\text {peak }}}}{L / 2 \lambda \mathrm{f}}
\end{aligned}
$$

where $\psi_{x_{\text {peak }}}$ and $\psi_{y_{\text {peak }}}$ are the peak values of the phase ramp along $f_{x}$ and $f_{y}$ respectively and $\mathrm{f}$ is the focal length of $L 1$ from the 4 -f system of Figure 2.4. Therefore, the amount of shift is given by:

$$
\begin{aligned}
& S_{x}=\frac{\lambda \mathrm{f}}{L} \frac{\psi_{x_{p e a k}}}{\pi} \\
& S_{y}=\frac{\lambda \mathrm{f}}{L} \frac{\psi_{y_{\text {peak }}}}{\pi}
\end{aligned}
$$

It can be seen that for a given 4 -f system, the peak phase values set the amount of the spatial shifting of the wavefront in the detector plane. Since SLM's have a finite range of programmable phase shift, $\psi_{\max }$, that is optimized for a specific range of wavelengths, it might seem that the spatial shifting would also be limited by that; however, a phase ramp can be wrapped as shown in Figure 2.7 to form a blazed grating that virtually extends the phase ramp slope beyond $\psi_{\max } / L$. In practice, this can be done to a certain extent because as the number of phase ramps in the blazed pattern increases, the pixelization of the SLM would increasingly distort the wavefront because the ramps would become less and less linear and take on the shape of a staircase which will produce undesired diffraction orders.

Another way of creating sheared wavefronts is by using two phase ramps of the 


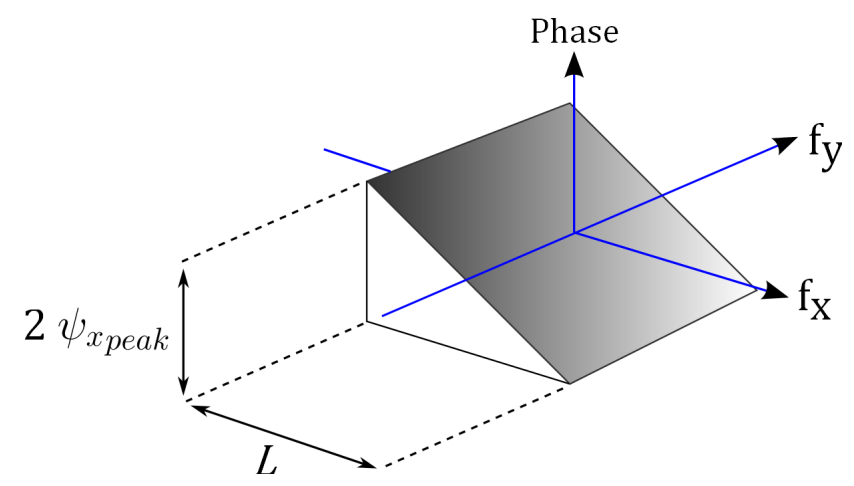

Figure 2.6: Two-dimensional phase ramp on the frequency plane with its center at the origin of the coordinate system.

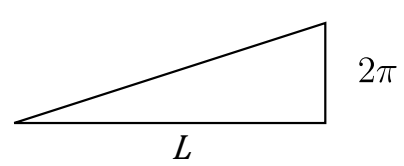

(a)

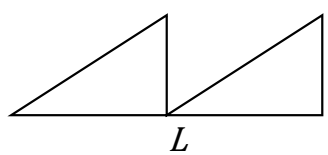

(c)

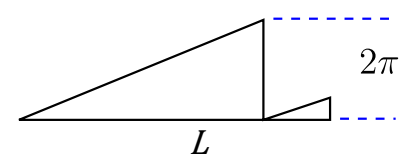

(b)

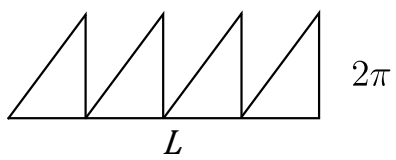

(d)

Figure 2.7: Wrapped phase patterns (also known as blazed patterns) extending the maximum phase retardation for a given SLM dimension, $L$. The blazed pattern number of cycles for these patterns are (a) $T=1$, (b) $T=1.25$, (c) $T=2$, and (d) $T=4$.

same slope sign but with different slope amounts instead of using opposite sloped phase ramps. Figure 2.8 illustrates this concept. The only difference here is that the shearing is not symmetric about the original wavefront's propagation vector but it is offset by the mean distance of the displacements caused by the blazed patterns. This has practical implications for reflectance mode SLM's as there always would be some amount of zeroth-order (specular) reflection from the reflecting surfaces of the device and this way it can be blocked or deflected away from the detector by steering the desired sheared beams away from it.

Regardless of the method used, two phase patterns are needed to create the shear- 


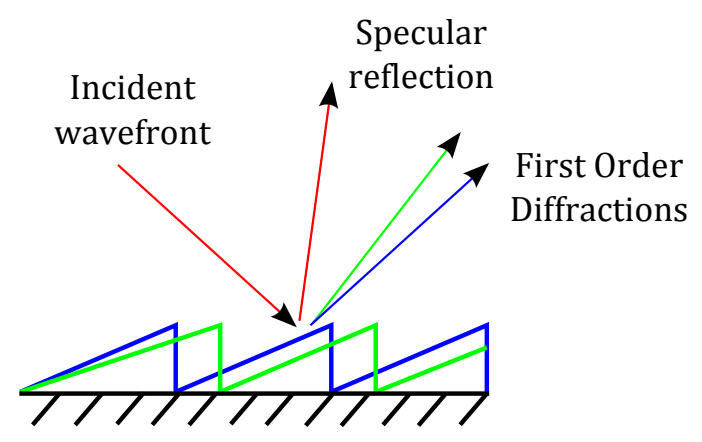

Figure 2.8: Two (blue and red) blazed phase patterns of the same phase slope sign used for steering the sheared wavefront away from the specular reflection component. The first order diffraction component of the blazed pattern with the larger slope (blue)

ing effect but implementing them in the 4-f system with only one phase-only SLM is problematic. This is because it would require the presence of two independent SLM's with two phase ramp functions at the same exact location or simultaneously two different values for each pixels of the SLM. For example Figure 2.9 shows a pair of phase planes of Eq. 2.17 needed for shearing along the x-axis. From this, it is evident that the exact realization of these two patterns with a single SLM is not possible. A common first-approach mistake is to sum these two patterns into one and apply it to the SLM but this produces:

$$
e^{i\left(\psi_{1}+\psi_{2}\right)}=e^{i \psi_{1}} e^{i \psi_{2}}
$$

which is just a phase shift applied to the Fourier transform of the input wavefront not two different phase shifts to two independent copies of the input. The main principle at work here is that the summation of the two exponential terms in Eq. 2.17 implies two independent SLM's. The next section explores combining these two phase patterns into one by a random selection method to emulate the effects of this summation operation with only one phase-only SLM. 


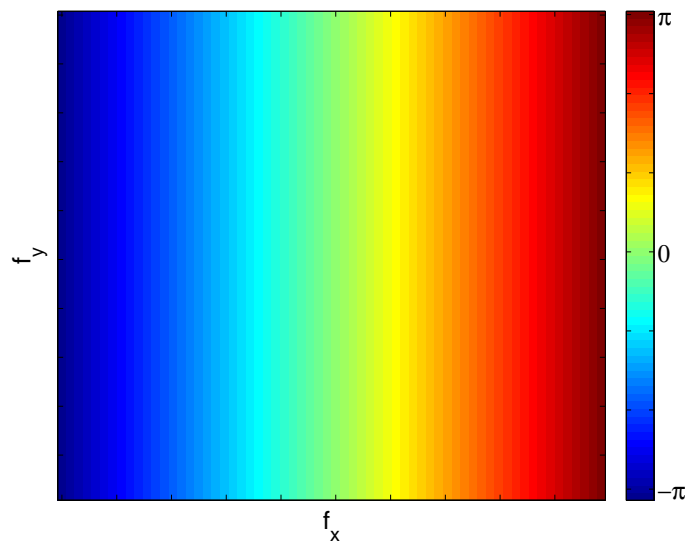

(a) Phase ramp with slope $=+\pi / f_{x_{\max }}$.

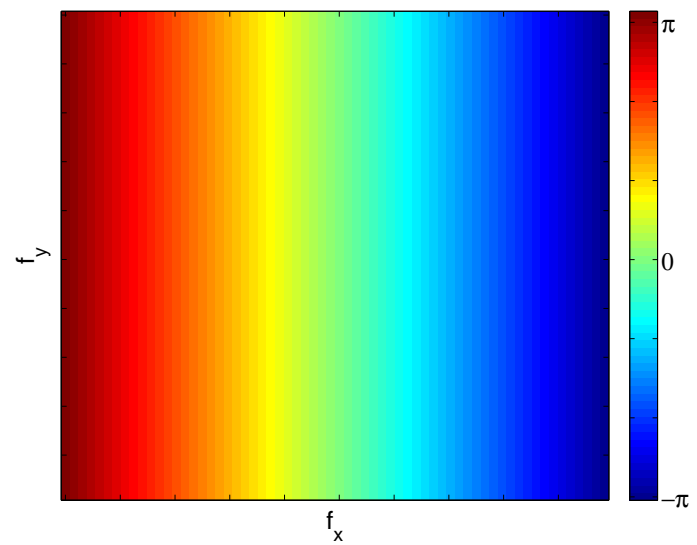

(b) Phase ramp with slope $=-\pi / f_{x_{\max }}$.

Figure 2.9: Two phase planes with opposite slopes along the x-axis.

\subsection{Phase Pattern Combination by Random Selection}

Here the objective is to have a selection method where half of SLM's pixels are selected randomly and assigned values from the corresponding pixels of the first phase pattern and the remaining half of SLM's pixels are assigned values from corresponding pixels of the second phase pattern. Pixel selection is done using a random binary matrix with uniform distribution and size equal to that of the pixels in the SLM. The uniform distribution of this matrix ensures equal numbers of 0's and 1's pixels. Figure 2.10 illustrates the selection and combination algorithm based on a random binary matrix. Figure 2.11(a) shows a numerically generated random binary mask with uniform distribution used to combine patterns of Figure 2.9 resulting in the pattern of Figure 2.11(b).

Mathematically the expression for the transfer function of the 4-f system becomes:

$$
H_{S L M}=e^{i\left(R \psi_{1}+\bar{R} \psi_{2}\right)}
$$

where $R$ is the two dimensional function of the random binary matrix and $\bar{R}$ is the 


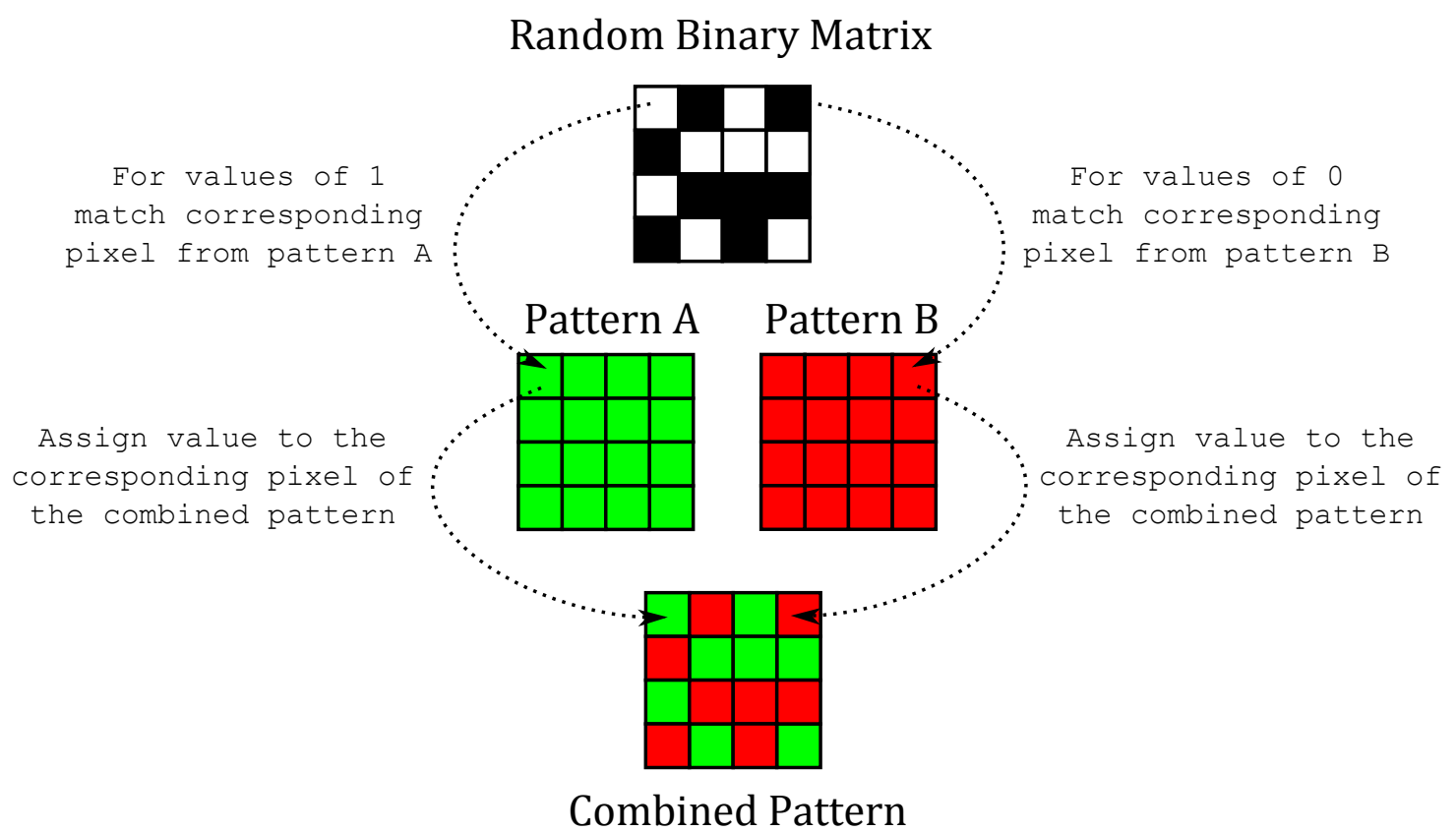

Figure 2.10: Random pattern combination algorithm.

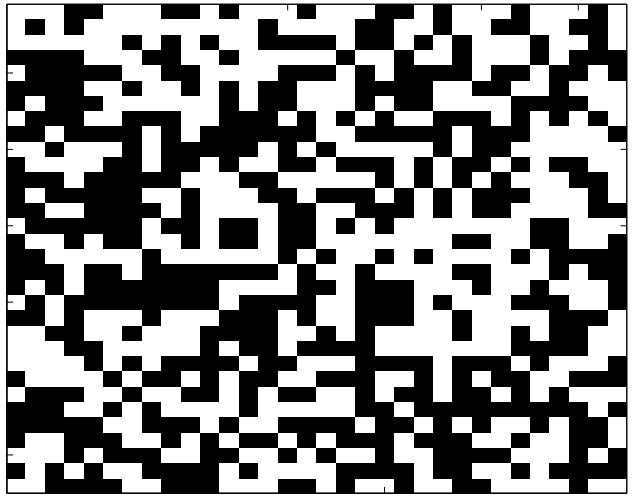

(a) Uniform random binary mask.

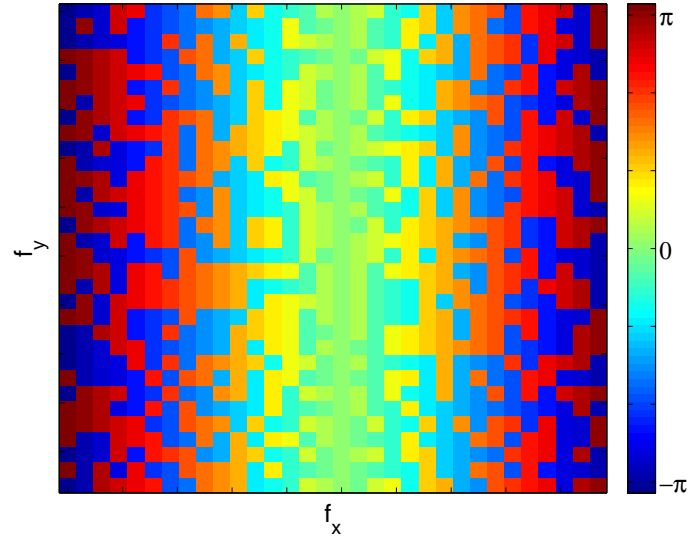

(b) Randomly combined ramps.

Figure 2.11: A zoomed-in section of the phase ramps of Figure 2.9 combined by random selection process.

complement of $R$. Clearly, combining two blazed patterns in this manner is not a one-to-one replacement of two independent blazed patterns in the DIC system and some added distortion is the consequence of this approach. This distortion can be improved by performing DIC in two steps. Once with the transfer function of Eq. 
2.25 and once with its complement:

$$
\bar{H}_{S L M}=e^{i\left(\bar{R} \psi_{1}+R \psi_{2}\right)}
$$

and then averaging together the resulting DIC images from each transfer function.

The analyitical treatment of shearing with the SLM function given by Eq. 2.25 is presented in Appendix B with the intensity expression given by Eq. B.9.

\subsection{Phase Shifting Interferometry with a 4-f System}

In a 4-f system, phase shifting interferometry can be performed by shifting the SLM's pattern laterally. For example, for a SLM pattern with periodic behavior such as a sinusoidal or a sawtooth (blazed) pattern of period $M$ pixels and $T$ number of cycles on an $N$ by $N$ pixel SLM, a circular shift of $M / 4$ pixels along the pattern's tilt

direction is equivalent of a lateral phase shift of $\pi / 2$. Figure 2.12 shows four examples of a laterally phase shifted cosine pattern.

A pattern phase shift of $\theta$ changes the transfer function of the DIC 4 -f system given by Eq. 2.12 to:

$$
H_{S L M}=\frac{1}{2}\left[e^{i 2 \pi\left(f_{x} S_{x}+f_{y} S_{y}\right)} e^{i \theta}+e^{-i 2 \pi\left(f_{x} S_{x}+f_{y} S_{y}\right)} e^{-i \theta}\right]
$$

The output fields are then given by:

$$
E_{\text {out }}(x, y)=\mathcal{F}\left\{\mathcal{F}\left\{e^{i \Phi(x, y)}\right\} H_{S L M}\right\}
$$


with the captured intensity image:

$$
I(x, y)=E_{\text {out }}(x, y) E_{\text {out }}^{*}(x, y)
$$

that yields a similar expression to Eq. 2.10:

$$
I(x, y)=\alpha+\beta \cos (\Delta \Phi+2 \theta)
$$

This shows that twice the lateral phase shift value of the SLM pattern appears in the intensity expression; therefore, if, for example, a phase shift of $\pi$ is required for PSI the SLM pattern should be shifted by $\pi / 2$.

In Appendix A, this concept is extended to an 8-step PSI process to extract $\Delta \Phi$
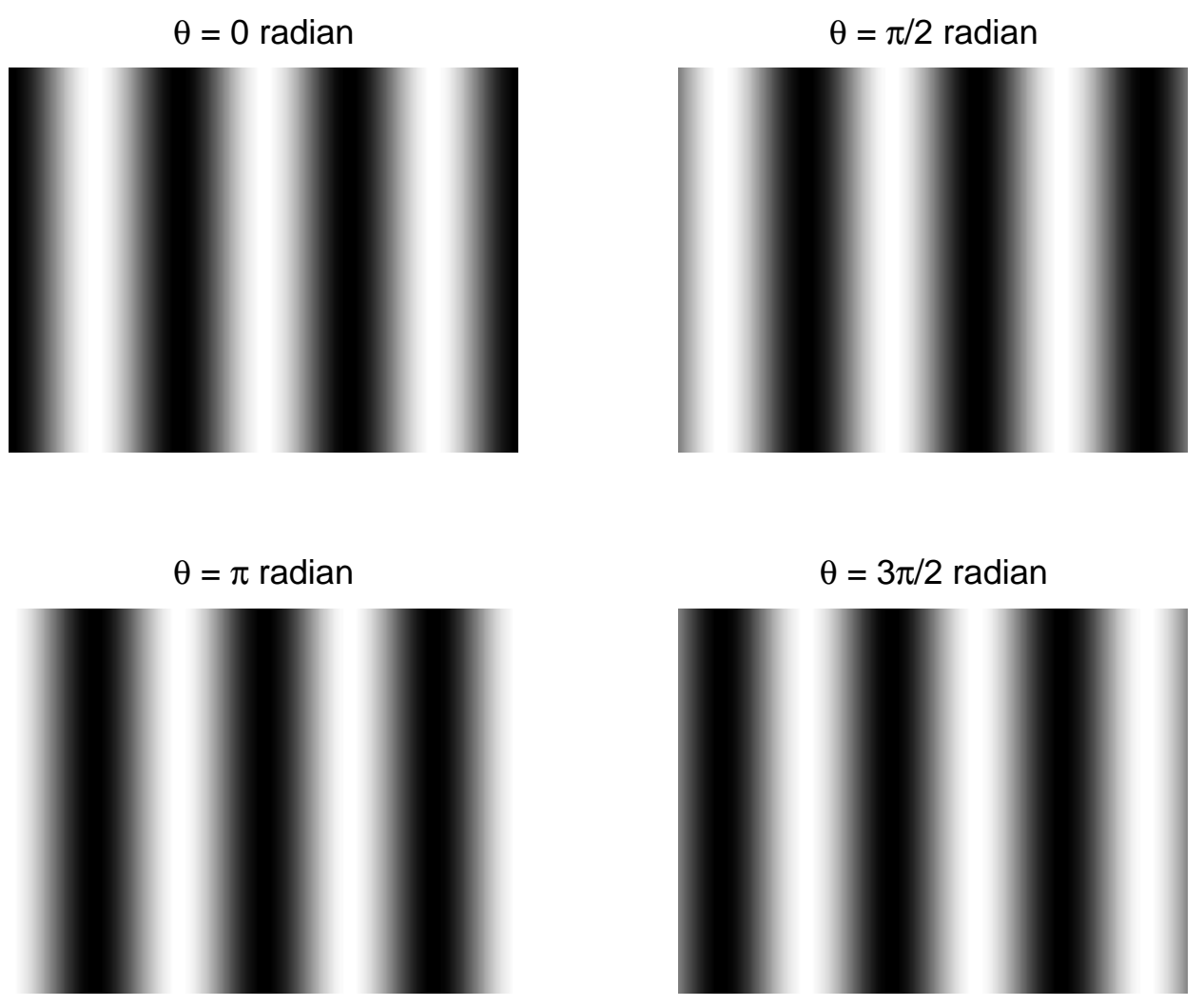

Figure 2.12: Lateral phase shifting of a cosine pattern. 
when a zeroth order diffraction component is also present at the detector plane along with the two sheared fields.

The implications of this result is one of the key advantages of the Fourier DIC imaging technique with an SLM discussed in this chapter because it eliminates the need for external hardware such as multiple phase plates (formally known bias retardation in conventional DIC microscopes) to introduce phase stepping into the system. 


\section{Chapter 3}

\section{Numerical Simulations and Analysis}

This chapter is an accompaniment for the previous chapter to provide visualization and verification for some of the theoretical concepts developed. It also serves as a reference tool to assist with verification of the lab measurements. First, an overview of the simulation method implementing the 4 -f system and the test object are given followed by examples and analysis from three cases for the SLM function: 1) amplitude cosine for the ideal DIC, 2) cosine transmittance, and 3) randomly combined ramps. The simulation programs are written in MATLAB and the scripts for each of these cases that are used to generate the results in this chapter are available in Appendix C. 


\subsection{Simulation of the 4-f System and PSI}

The signal processing system implemented for the simulations is shown in Figure 3.1 where the two-dimensional fast Fourier transform (FFT) of the input fields, $E_{o b j}$, given as a two-dimensional matrix is taken and multiplied by the SLM function that is generated in the frequency domain. The second FFT gives the output fields, $E_{\text {out }}$, and the intensity is calculated by multiplying the output fields matrix by its complex conjugate. PSI is performed by repeating this process in a loop for each of the SLM function's lateral phase offset. The intensity matrix obtained for each step, $I_{j}$, is then used to calculate the phase difference, $\Delta \Phi$, using Eq. 2.11. The simulations only

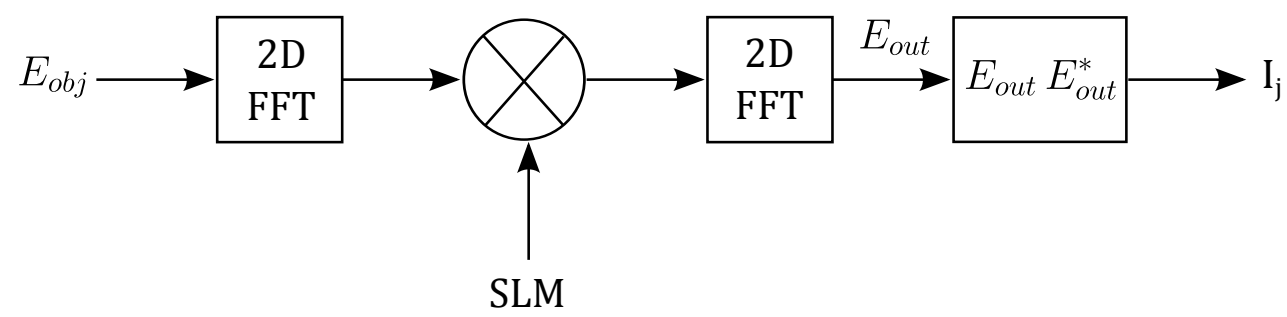

Figure 3.1: Block diagram of the system simulating the 4-f system.

differ in the SLM functions and the PSI method used (Table 3.1). No magnification is introduced in the system. The object geometry selected for $E_{o b j}$ in the simulations has radial symmetry and therefore in the examples of this chapter only shearing along the $\mathrm{x}$-axis is considered; however, the simulation program does include a shear rotation angle parameter.

Table 3.1: SLM Functions of the Simulation Cases

\begin{tabular}{l|c|c}
\multicolumn{1}{c|}{ Case } & SLM Function & Sec. \\
\hline Ideal & $\cos \left(2 \pi s f_{x}+\theta_{j}\right)$ & 2.5 \\
\hline Cosine Transmittance & $\frac{1}{2}+\frac{1}{2} \cos \left(2 \pi s f_{x}+\theta_{j}+\theta_{k}\right)$ & 2.4 \\
\hline Randomly Combined Ramps & $A_{0}+\left(1-A_{0}\right) e^{R\left(i 2 \pi s f_{x}+\theta_{j}+\theta_{k}\right)+\hat{R}\left(-i 2 \pi s f_{x}+\theta_{j}+\theta_{k}\right)}$ & 2.5
\end{tabular}




\subsection{Simulation of the Phase Object}

The object's phase function used in the simulations is a semi-spheroid formed on a normalized $512 \times 512$ symmetric coordinate matrices $X$ and $Y$ whose axes values range from $-1 / 2$ to $+1 / 2$ with interval values of $1 / 512$. The phase function of the object is given by:

$$
\Phi_{o b j}=\kappa 2 \pi \Re\left\{\sqrt{R^{2}-\frac{X^{2}+Y^{2}}{R}}\right\}
$$

where $R$ is the lateral radius, $\kappa$ is a scaling factor determining the weakness of the phase function by scaling the height of the semi-spheroid, and $\Re$ denotes the real part. For a given $R$, larger $\kappa$ values produce larger heights for the semi-spheroid and therefore the phase difference between two adjacent pints on the object increases resulting in a "stronger" phase effect on an incident plane wave. Figure 3.2a shows the two-dimensional graph of the phase function with its cross section view in Figure $3.2 \mathrm{~b}$. The object fields are simulated as $E_{o b j}=e^{i \Phi_{o b j}}$ which is a wavefront with uniform amplitude of 1 and phase given by $\Phi_{o b j}$.

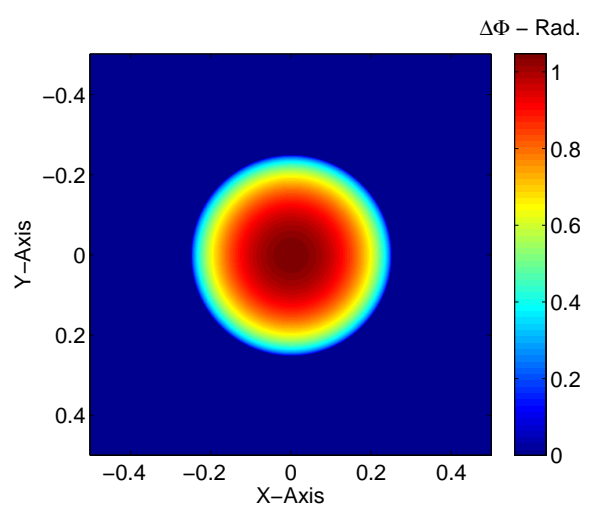

(a)

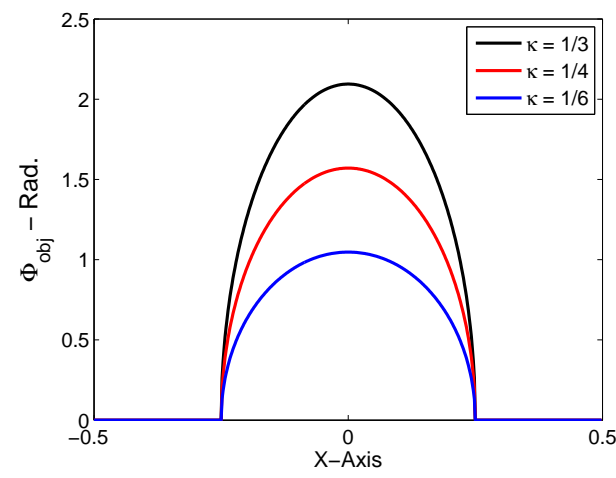

(b)

Figure 3.2: (a) Two-dimensional plot of the phase function of the object with $R=1 / 4$ and $\kappa=1 / 6$. (b) Cross section plots of the phase function of the object with with $R=1 / 4$ and $\kappa=1 / 3$ (black), $1 / 4$ (red), and $1 / 6$ (blue). 


\subsection{Case I: Ideal Fourier DIC}

The ideal case was derived from applying the Fourier shift theorem to create two shifted copies of the input fields at the output with a cosine amplitude SLM function. Physical implementation of this is impossible since it requires negative field amplitudes but this ideal case serves as a comparison for other methods. Figure 3.3 shows the intensity images resulting from the four-step PSI for $s=a / N=1 / 512$ that sets the shear distance to two pixels. Images for each offset manifests the typical edge enhancement characteristics of a DIC image. The calculated phase difference, $\Delta \Phi$,
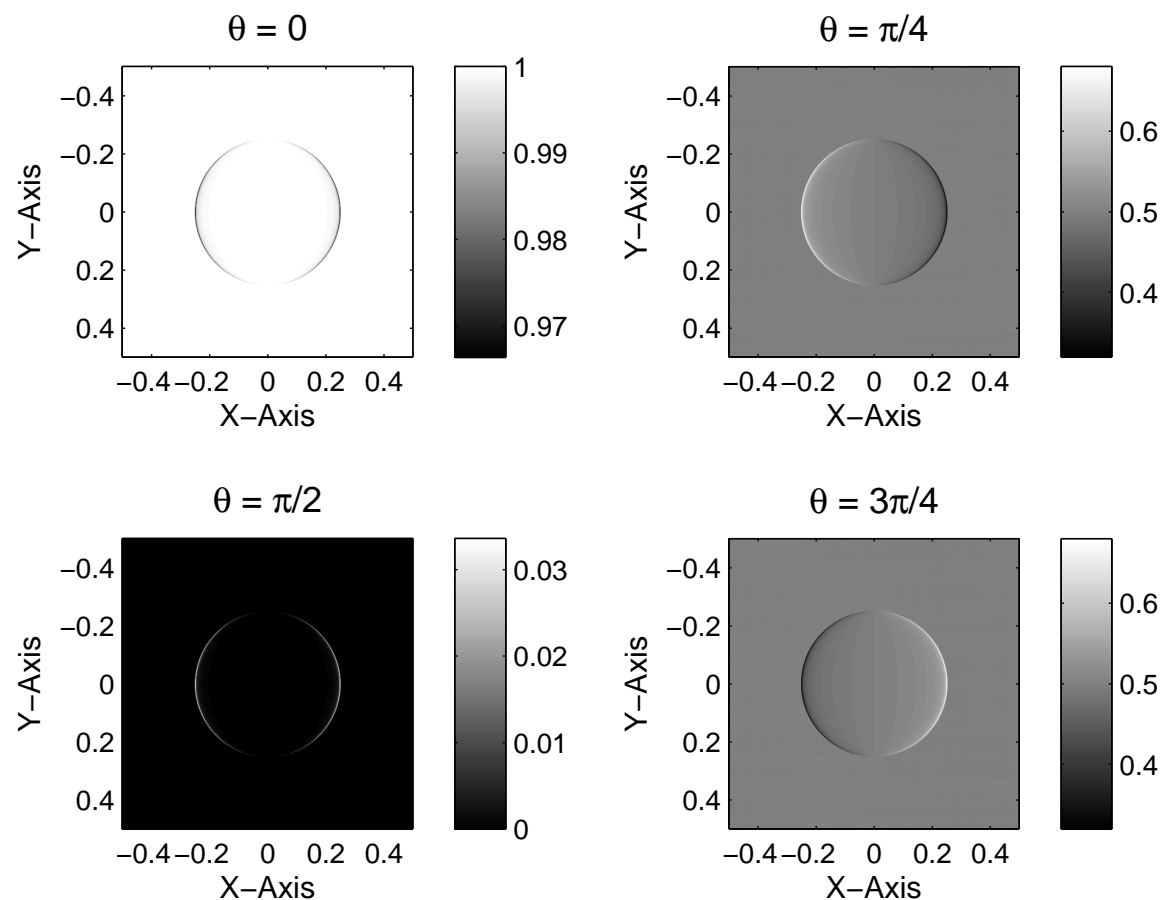

Figure 3.3: Intensity images from the four-step PSI with amplitude cosine SLM function.

from these four images is shown in Figure 3.4a. To validate this result, two copies of the phase function of the object matrix, $\Phi_{o b j}$ (Figure 3.2a), one shifted by one column to the right and the other shifted by one column to the left are subtracted from each other to obtain the derivative of $\Phi_{o b j}$ along the x-axis over two pixels. Figure 3.4b 
shows the plots of the cross section taken from the middle row. The two results are identical. This verifies the shearing operation by employing the shift theorem as well as the PSI method derived in section 2.6

Figure 3.5 shows the comparison of the cross section plots of $\Delta \Phi$ for 3 different shear amounts along the x-axis. As expected, the phase difference increases for larger shear distances. The zero crossing point indicates the location of the peak of the phase object where the slope of its topography is zero.

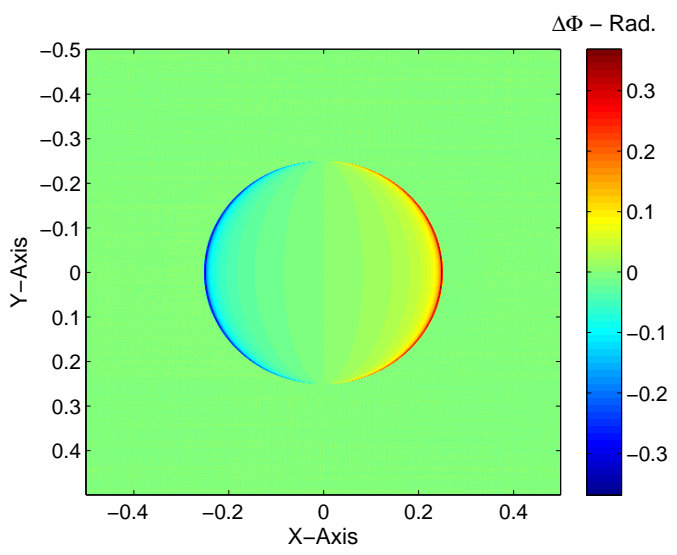

(a)

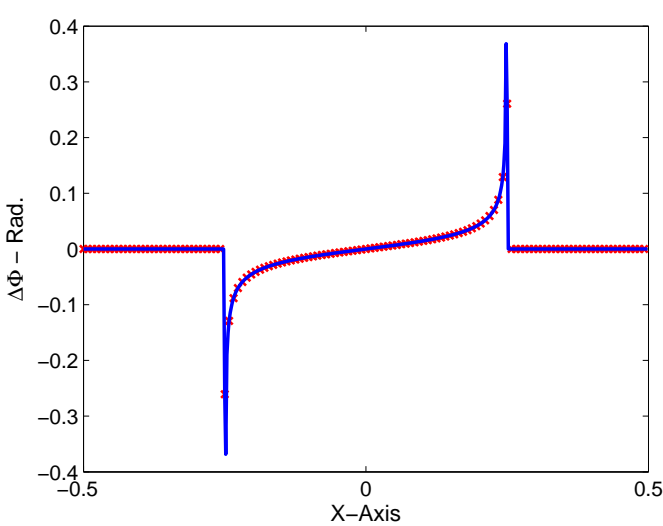

(b)

Figure 3.4: (a) $\Delta \Phi$ from the ideal Fourier DIC case calculated from the four intensity images of Figure 3.3. (b) Cross section view of $\Delta \Phi$ (blue) and direct phase function derivative over 2 pixels (red).

MATLAB scripts for the simulations of this section are available in Appendix C.1. 


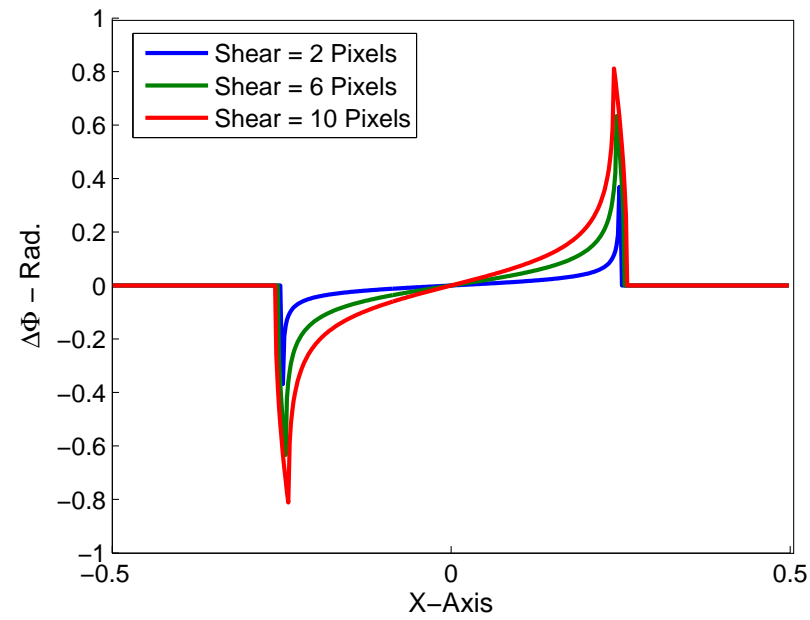

Figure 3.5: Cross section view of $\Delta \Phi$ with different shear amounts.

\subsection{Case II: Cosine Transmittance}

The cosine transmittance filter function produces three shear terms (Eq. A.5) at the detector plane due to the interference of the diffracted orders with the zeroth order and therefore recovering the phase difference is not possible with the conventional PSI techniques. Figure 3.7a shows the phase difference map with $s=1 / 512$ calculated from the four-step PSI with the cross section view in Figure 3.7b. This result does in fact resemble the characteristics of the result obtained from the ideal case and may be sufficient for qualitative analysis but as shown in Figure 3.7b it yields an incorrect phase difference map. The eight-step PSI developed in Appendix A to eliminate this error is a modification of the four-step PSI where for each of the phase offset vales of the filter function, $\theta_{j}$, two intensity images are required one with the secondary phase offset term $\theta_{k}=0$ and another one with $\theta_{k}=\pi$. Figure 3.6 shows the simulated intensity images from this PSI method and Figure 3.8a is the resulting phase difference map with the cross section view in Figure $3.8 \mathrm{~b}$ where $\Delta \Phi$ is now identical to the ideal case. This validates the theoretical predictions of the eight-step PSI method. 

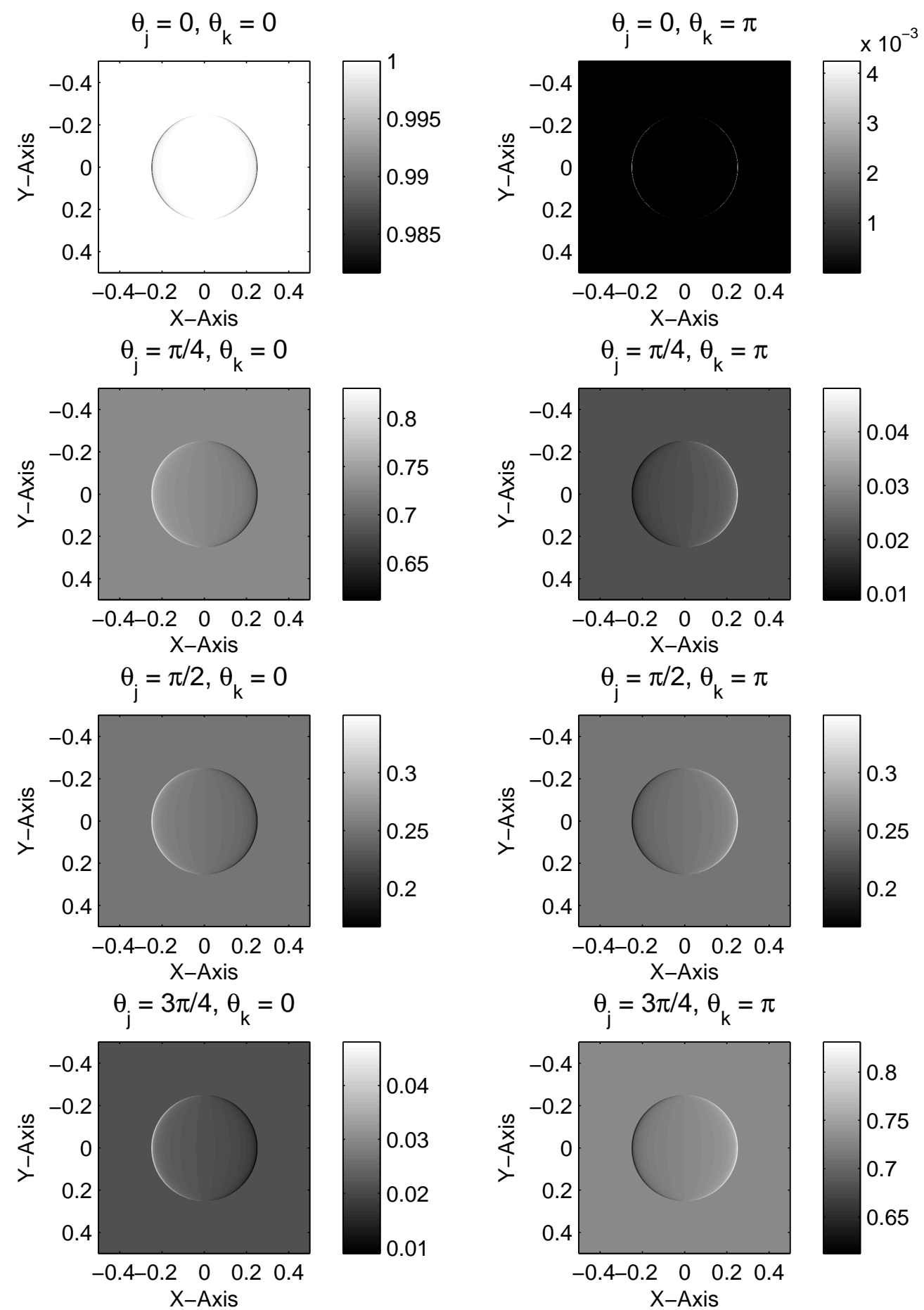

Figure 3.6: Intensity images from the eight-step PSI with cosine transmittance SLM function. 


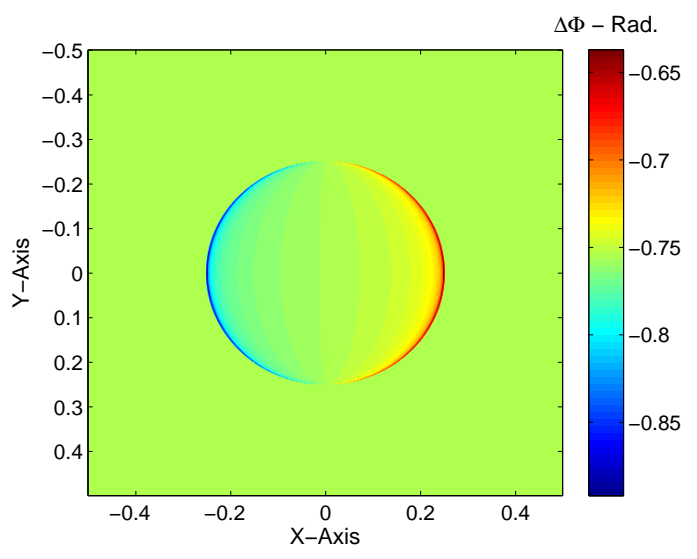

(a)

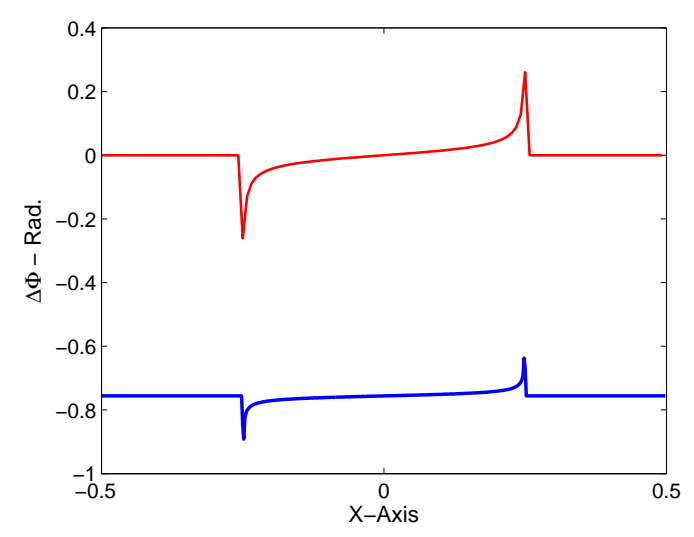

(b)

Figure 3.7: (a) $\Delta \Phi$ with cosine transmittance SLM function calculated from the four-step PSI. (b) Cross section view of $\Delta \Phi$ (blue) and direct phase function derivative over 2 pixels (red).

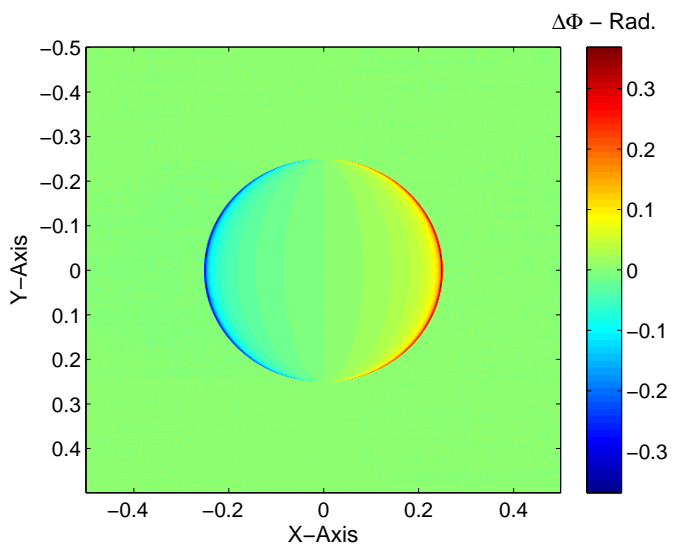

(a)

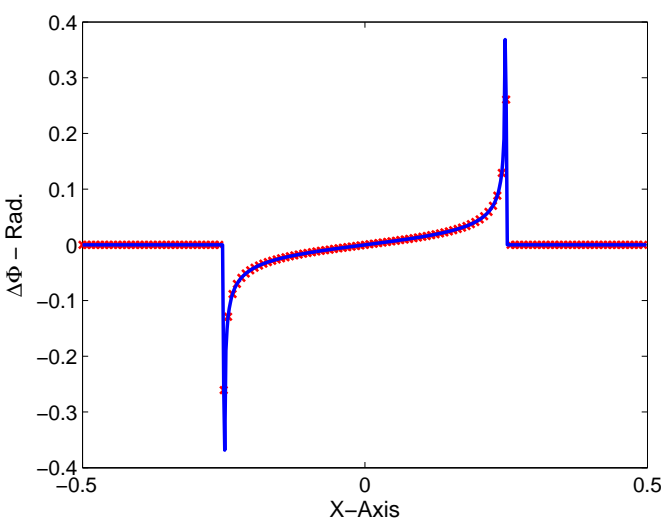

(b)

Figure 3.8: (a) $\Delta \Phi$ with cosine transmittance SLM function calculated from the eight-step PSI images of Figure 3.6. (b) Cross section view of $\Delta \Phi$ (blue) and direct phase function derivative over 2 pixels (red).

MATLAB scripts for the simulations of this section are available in Appendix C.2

\subsection{Case III: Randomly Combined Ramps}

Combining two phase ramps of opposite slopes by a random process aims to replicate the ideal DIC case on a phase-only SLM (section 2.5). Generation of noise 
as a result of introducing a random mask is expected in both the intensity images and the calculated phase difference map. To simulate the specular reflection from the surface of the SLM, an arbitrary small DC term $A_{0}=1 / 10$ is added to the SLM function (table 3.1)that produces a weak, un-diffracted copy of the input fields along with the two sheared fields in the detector plane; therefore, similar to the cosine transmittance function case, the 8-step PSI is required to extract $\Delta \Phi$. Figure 3.10 shows the DIC images for each of the eight steps with $s=1 / 512$. Each of these DIC images is an average of two images resulting from the randomly combined SLM function and its complement to reduce noise (see section 2.5 for details).

The DIC image $I_{j k}=I_{00}$ with $\theta_{j}=0, \theta_{k}=0$ shows similar edge enhancement features to the DIC image from the ideal case $I_{j}=I_{0}$ with $\theta_{j}=0$ (Figure 3.3) with some added small amount of distortion. From this it can be concluded that the pattern combination by random selection method does closely approximate the shearing operation of the ideal case. The remaining simulated DIC images with $\theta_{j} \neq 0$ are cluttered with severe distortion that consequently results in a highly distorted phase difference map shown in Figure 3.9a. The cross section of the calculated phase difference is compared with the ideal case in Figure 3.9b. In areas around the edges of the object the shape of the phase difference approximately matches the expected case. Simulation iterations with with different phase object dimensions, various $\kappa$ values, larger matrix sizes $(N)$, different shear distances $(s)$ and angles, as well a case without the DC term in the SLM function showed that this distortion is insensitive to these parameters. Furthermore, the lab measurement results from this method do exhibit distortions but not with such strength and large signature present in the numerical simulation results. Therefore, it is inconclusive at this point as to what causes the severely distorted DIC images generated with phase offsets other than 0 and further investigation is needed. 
MATLAB scripts for the simulations of this section are available in Appendix C.3 and Appendix C.4

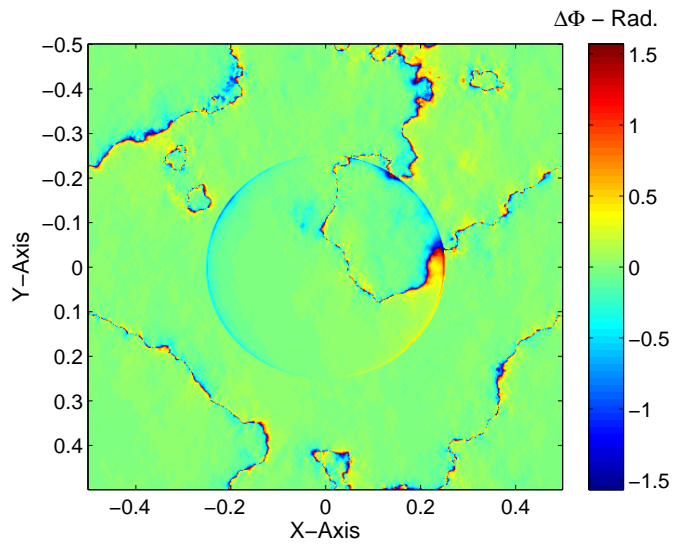

(a)

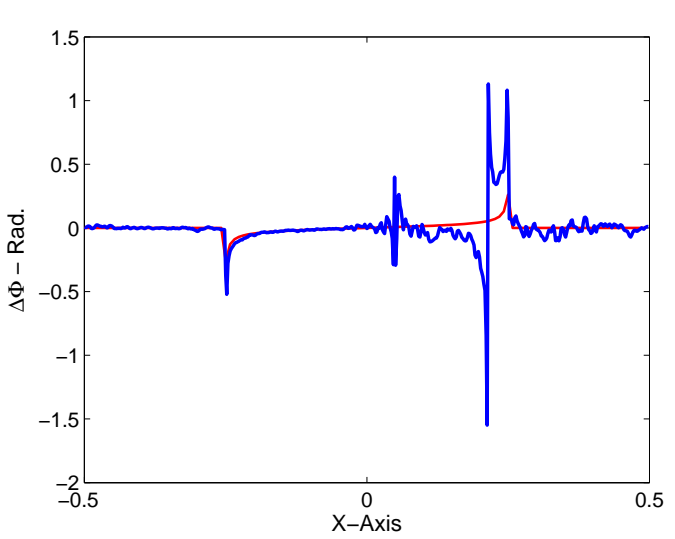

(b)

Figure 3.9: (a) $\Delta \Phi$ with randomly combined ramps SLM function calculated from the eight-step PSI images of Figure 3.10. (b) Cross section view of $\Delta \Phi$ (blue) and direct phase function derivative over 2 pixels (red). 

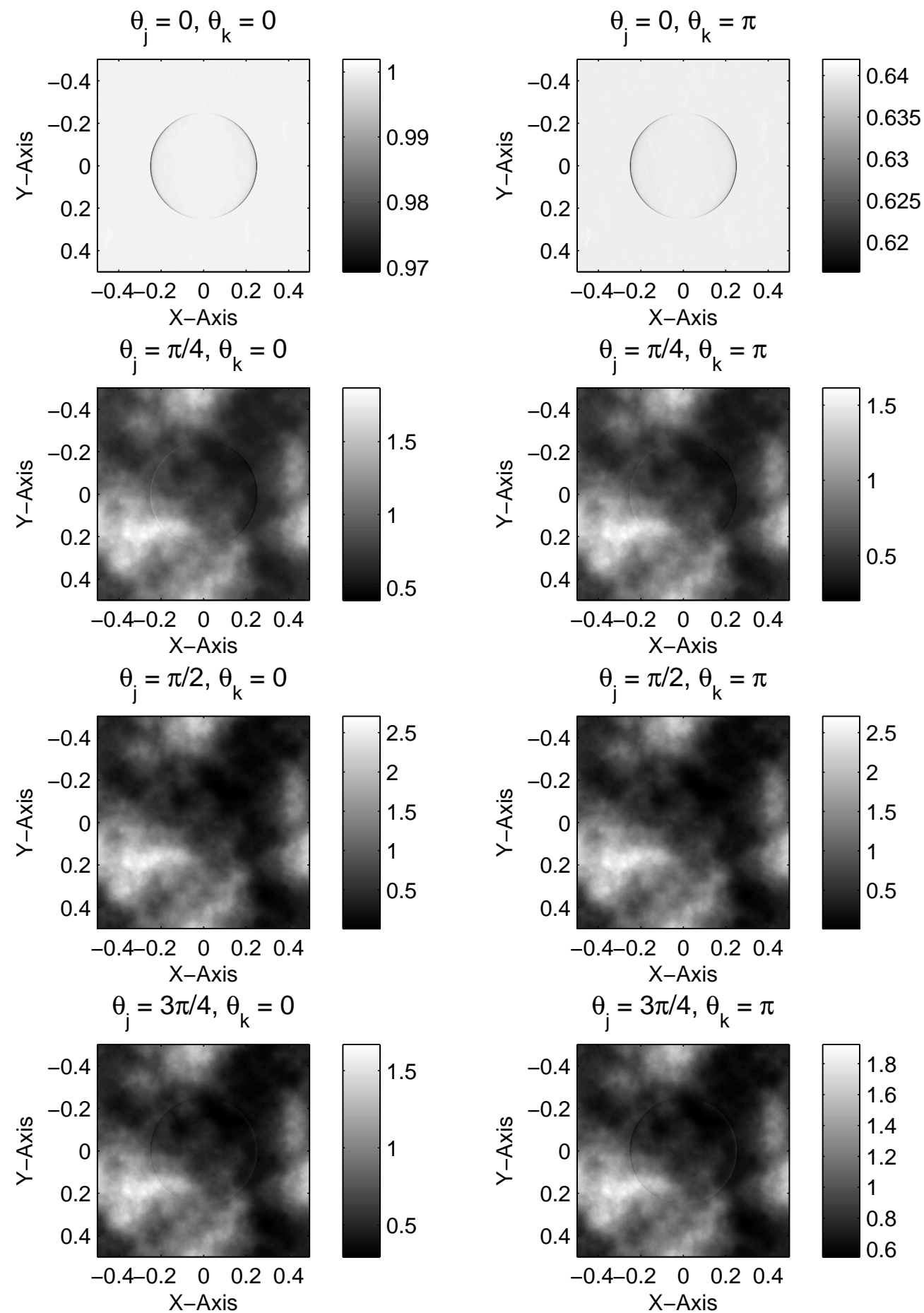

Figure 3.10: Intensity images from the eight-step PSI with randomly combined ramps SLM function. 


\section{Chapter 4}

\section{Experimental Configuration}

This chapter provides information about the structure and operation of the custom-

built DIC microscope where the shearing by two phase ramps combined by random selection process and PSI are implemented in a close-loop imaging system controlled by a computer. 


\subsection{Hardware Design}

The configuration design is based on [40] in which a benchtop optical chain is arranged to form a microscope with its functionality extended to a 4-f system with a phase-only SLM and a CCD. Figure 4.1 shows the schematic of the configuration. The CCD and the SLM are connected to a PC and they are controlled with a custom MATLAB software application that loads numerically generated patterns onto the SLM and retrieves images from the CCD.

Since the SLM's operation is optimized for a specific spectrum range $(615 \mathrm{~nm}$ to $700 \mathrm{~nm})$, a $60-\mathrm{mW}$ diode laser with wavelength of $660 \mathrm{~nm}$ coupled into a short-length optical fiber is used as the illumination source. To eliminate speckle the spatial coherence of the laser light needs to be destroyed. This is done by a rotating ground glass diffuser that is placed about $10 \mathrm{~cm}$ in front of the fiber to scatter and diffuse the light to the backside of a stationary diffuser that acts as the virtual illumination source for the microscope. The rotating diffuser is a custom apparatus in which the center of a ground glass is drilled and attached with a screw to a shaft that is connected to a gear assembly and a DC motor with a variable voltage supply. The angular speed of the rotating diffuser is manually adjusted while monitoring the live image stream from the CCD until the speckle noise is minimized.

The illumination aperture A1 is an iris diaphragm adjusted for an opening of about $1.5 \mathrm{~cm}$. L1 and L2 image the aperture to the back focal plane of OBJ1 that is a 10x objective lens with $\mathrm{NA}=0.25$. This objective lens acts as the condenser of the illumination path by Fourier transforming the light spot on its back focal plane to a wide, uniform beam at the object plane that is placed at OBJ1's front focal plane.

The front and back focal distances of the objective lenses are measured experimentally 
by placing them on the path of a collimated laser beam and measuring the distance where the beam is converged into a small dot. The back focal plane is found to be at the inside end of the barrel threads and the front focal distance was found to match the published working distance $(6.8 \mathrm{~mm})$. Figure 4.2 shows the locations of the focal planes of the objective lenses.

The section of the optical chain starting from the object plane to the CCD is essentially a 4-f modulation system with a relay optics arm. The spatial Fourier transform of the fields emerging from the object is formed on the back focal plane of OBJ2 that is relayed by L3 and L4 onto the SLM and L5 performs another Fourier transform projected on the CCD. 
L1, L2: $\quad f=120 \mathrm{~mm}, \mathrm{D}=38.1 \mathrm{~mm}$

L3, L4: $f=160 \mathrm{~mm}, \mathrm{D}=38.1 \mathrm{~mm}$

L5: $\quad f=220 \mathrm{~mm}, \mathrm{D}=38.1 \mathrm{~mm}$

D1: $\quad$ Rotating ground glass diffusor

D2: $\quad$ Static ground glass diffusor

A1: Adjustable circular aperture

OBJ1, OBJ2: $10 \mathrm{X}, \mathrm{NA}=0.25$

P1: $\quad$ Polarizer

CCD: $\quad 1200 \times 1600 \mathrm{pxls}, 30 \mathrm{FPS}, 4.4 \mathrm{um}$

SLM: $\quad 512 \times 512,16-$ bit, $7.68 \times 7.68 \mathrm{~mm}$ active area,

$83.4 \%$ fill factor, wavelength $615-700 \mathrm{~nm}$
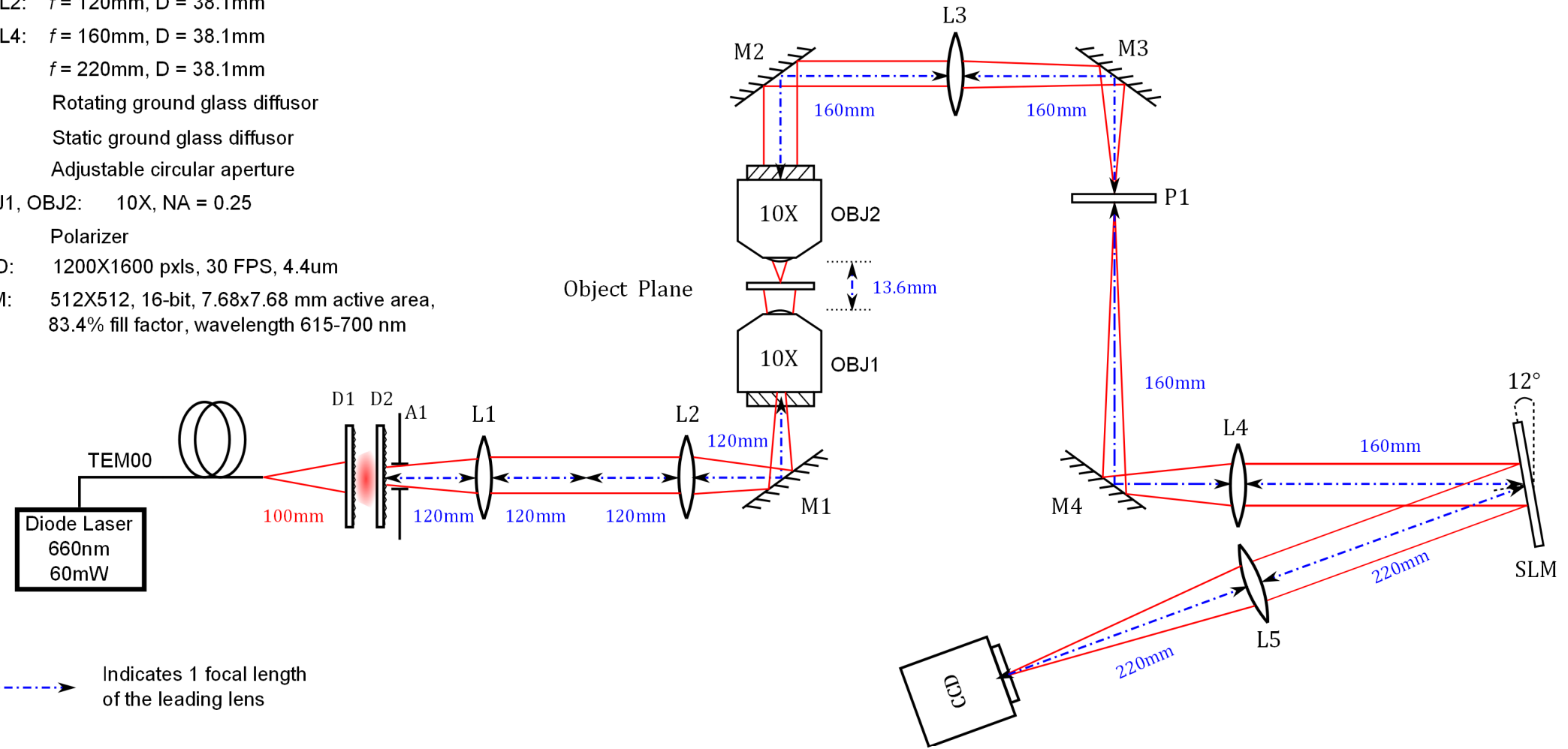

Figure 4.1: Schematic of the experimental configuration. 
OBJ2 is a matched pair of OBJ1 with 10x magnification and NA=0.25. The polarizer P1 aligns the fields along the fast axis of the SLM to ensure phase-only modulation.

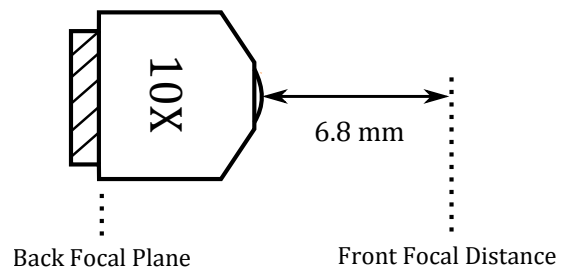

Figure 4.2: Relative locations of the front and back focal planes of the objective lenses.

The SLM pattern files are 512x512 16-bit unsigned integers in TIFF format where for each pixel the phase shift range of 0 to $2 \pi$ is encoded between integer numerical values of 0 to $2^{16}$. The MATLAB SDK supplied with the SLM includes a library of functions to interact with the device, a look-up table (LUT) calibration file specific to the product's serial number, and an example GUI script. The LUT file is loaded to the SLM prior to the loading of the pattern files for all experiments of this work. Since the SLM used in the configuration is a reflectance mode device, normal incident angle of the light is optimum for minimum cross talk between the adjacent pixels of the SLM. The manufacturer was consulted and it was recommended that for incident angles less than $15^{\circ}$ no re-calibration is needed and the original LUT is sufficient. Therefore, the SLM is mounted on a rotary translation stage and the tilt angle is adjusted to about $12^{\circ}$.

The CCD is a lens-less 1600 pixels horizontal by 1200 pixels vertical image sensor camera with unit cell dimension of $4.4 \mu \mathrm{m}$ square. Image capturing is done using commands from MATLAB's Image Acquisition toolbox library. To reduce image noise, every image used in the experiments is an average over five consecutive captures.

Figures 4.3, 4.5, and 4.6 show the pictures of the experimental configuration. 


\subsection{Software Design}

The software application developed for this work initializes and powers up the SLM and initially forms a brightfield image by writing a blank pattern (all pixel values set to zero) on the SLM which converts the SLM to simply a reflecting surface and captures it with the CCD. This brightfield image is used as the reference for visual comparison to the DIC images. Next using MATLAB's sawtooth function, it creates two $512 \times 512$ blazed patterns with opposite slopes and peak values of $2^{16}-1$ ( $2 \pi$ phase shift) with specified number of cycles, lateral phase shift angle, and rotation angle. Then it combines the two blazed patterns by random selection process as explained in section 2.5 to form the combined pattern $(\mathrm{RCP})$ and its complement pair (CRCP). The RCP and CRCP are loaded to the SLM and the DIC images resulting from each one of these two patterns is captured and averaged to form the final DIC image. The 8-step PSI is implemented by repeating this process from pattern generation to DIC image capture for each of the pattern's lateral phase shift that contains two components: $\theta_{j k}=\theta_{j}+\theta_{k}$ (Appendix A). $\theta_{j}$ is stepped through $0, \pi / 4, \pi / 2$, and $3 \pi / 4$ and for each of these steps two DIC images are captured. One with $\theta_{k}=0$ that produces the DIC image $I_{j k}=I_{j 0}$ and another one with $\theta_{k}=\pi$ that produces the DIC image $I_{j k}=I_{j 1}$. Finally, the summation $I_{j}=I_{j 0}+I_{j 1}$ gives the DIC image for every $\theta_{j}$ that is used to calculate the phase difference map of the object. The following expression summarizes the DIC image capturing progression for every $\theta_{j}$ :

$$
I_{j}=\frac{1}{2}\left(I_{j 0_{R C P}}+I_{j 0_{C R C P}}\right)+\frac{1}{2}\left(I_{j 1_{R C P}}+I_{j 1_{C R C P}}\right)
$$


The phase difference calculated using the atan2 function:

$$
\Delta \Phi=\operatorname{atan} 2\left\{I_{j=3}-I_{j=1}, I_{j=0}-I_{j=2}\right\}
$$

The random mask used for pattern combination is only generated once at the beginning of the program and used for all steps. Figure 4.3 shows the detailed algorithm implemented by the software application. 


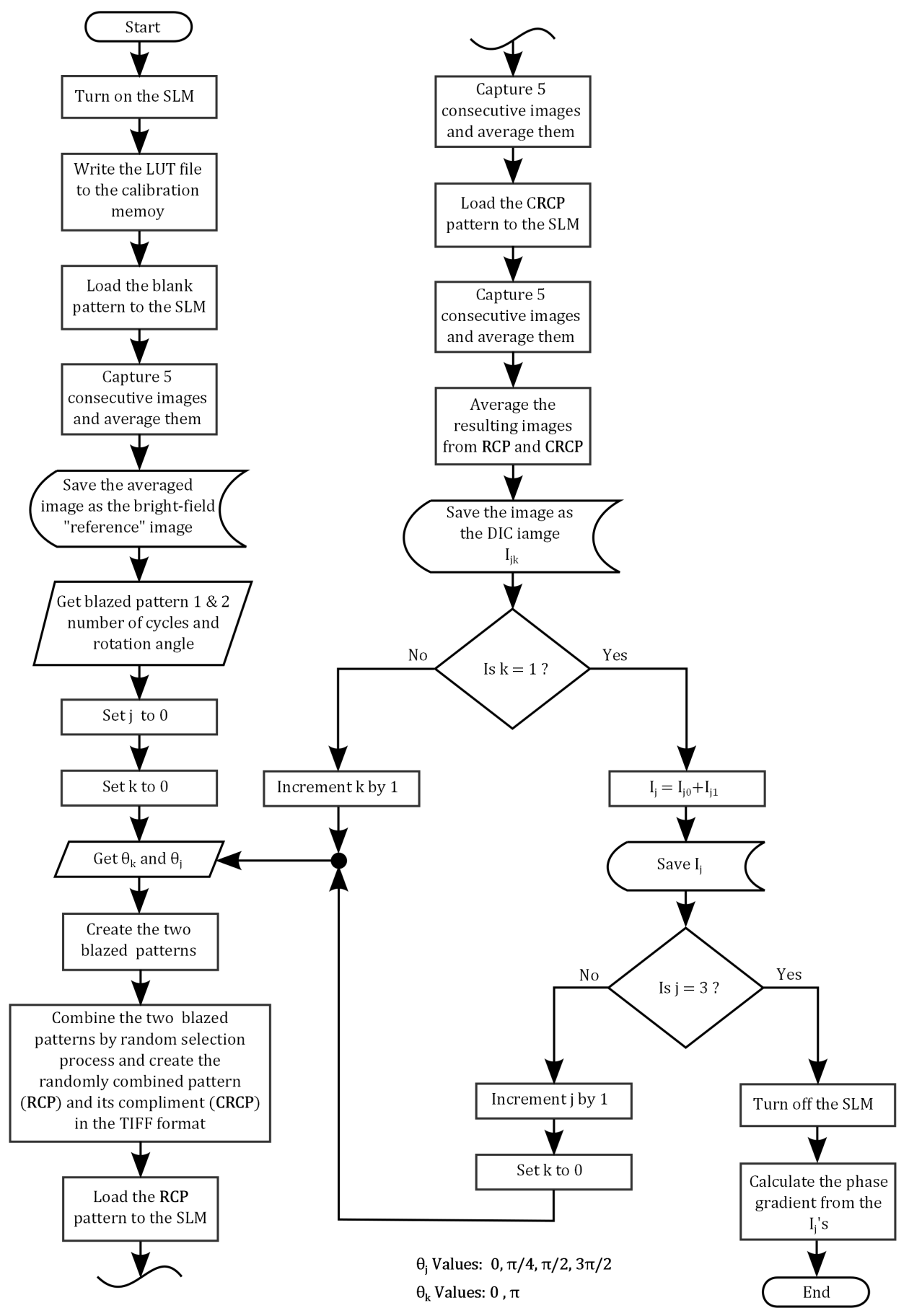

Figure 4.3: Program algorithm. 


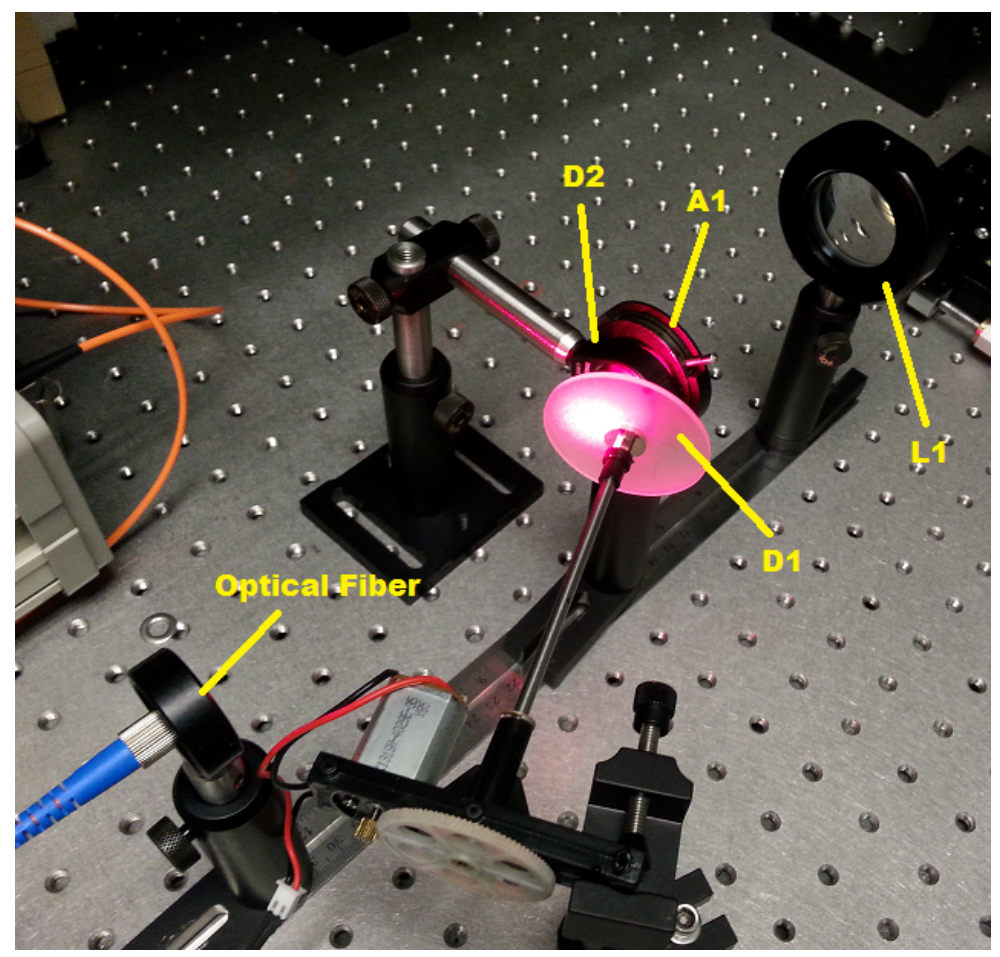

Figure 4.4: The illumination path of the experimental configuration.

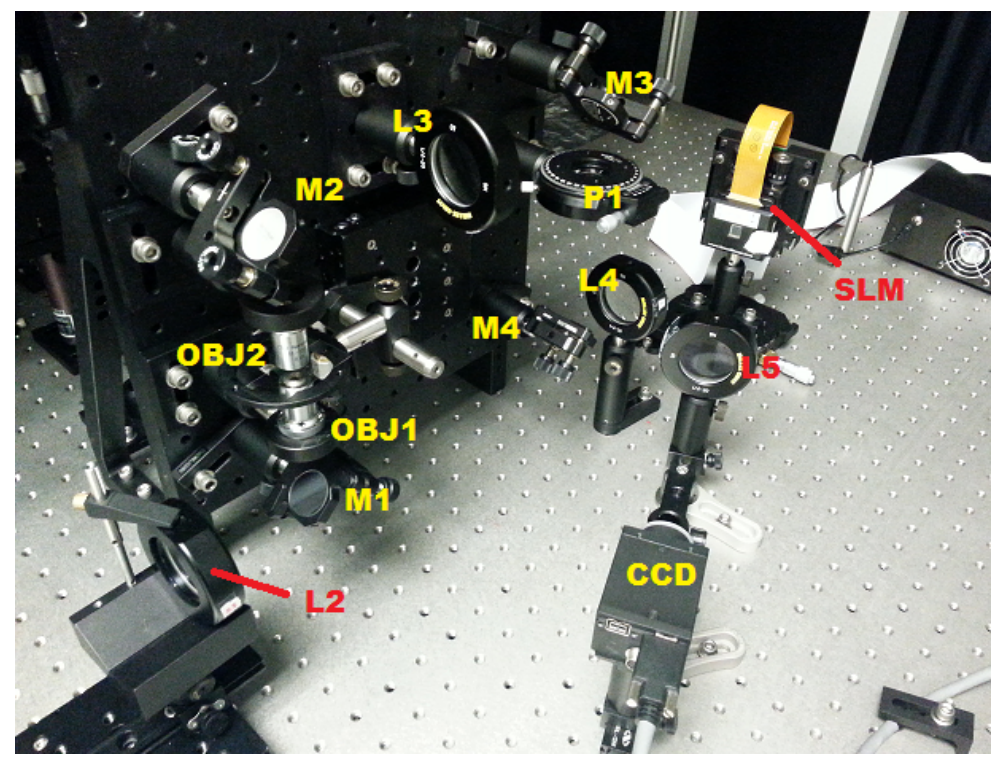

Figure 4.5: The imaging portion of the experimental configuration. 


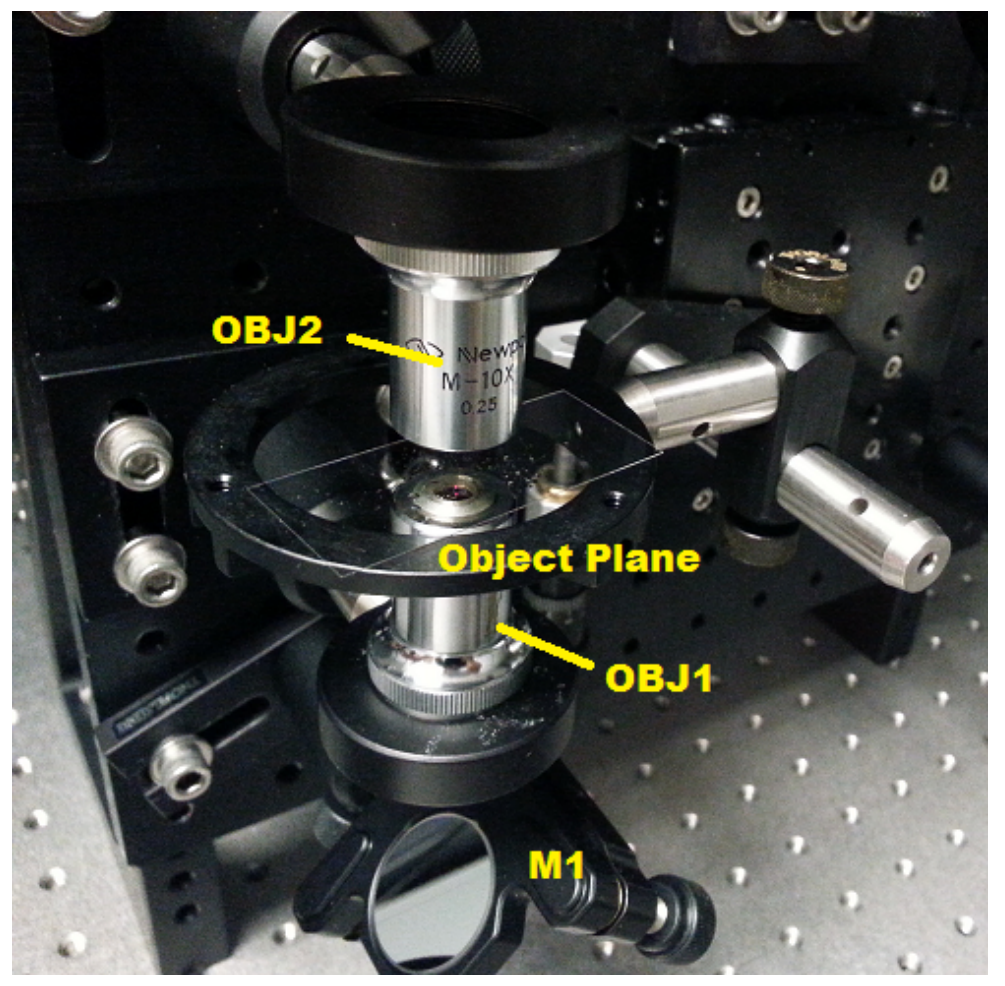

Figure 4.6: Close-up view of the object plane of the experimental configuration. 


\section{Chapter 5}

\section{Measurement Results and Analysis}

In this chapter, a series of measurements that are made to determine the shear amount as a function of the number of cycles in the blazed pattern as well as the effective magnification of the system are presented. The DIC images from PSI measurement steps along with the calculated phase difference map for a range of different shear distances and directions are followed along with data analysis and verification of the system's DIC and PSI operations. 


\subsection{System Characterization}

Two key parameters of the DIC microscope are its shear distance and shear direction. The shear direction is set by the rotation angle of the SLM pattern. The shear distance is set by the blazed pattern and the effective magnification of the system. The experimental methods and measurement results used to determine the shear amount and magnification of the system are presented in this section.

The shear amount of the microscope is controlled by the slope of the phase ramps applied to the SLM. In section 2.4, it was shown that this slope can be extended to beyond the maximum phase shift of the SLM over its side length by wrapping the phase ramp pattern to form a blazed pattern. Therefore, the number of blazed cycles (wrapped ramps), $T$, determines the spatial shift amount of the fields (Figure 2.7). To measure this relationship, displacement of the image of an object is tracked and compared to its original pixel location as $T$ of a single blazed pattern on the SLM is swept. Figure 5.1 shows the measurement results of this experiment for a blazed pattern with $T$ swept from 0.25 to 6.5 by 0.25 along the horizontal-axis. As expected, larger number of blazed cycles result in larger displacement amount. This relationship also has a strong linear behavior with the exception of some points where they are only off by one pixel from the linear curve. Since the detectable displacement resolution is also only one pixel, it is concluded that the assumption of linearity is an accurate approximation for $T>0.75$. Furthermore, for $T=0.25$ and $T=0.5$ the displacement is too small to be detected by the CCD. Maintaining the same assumption of linearity for $T<0.75$, the amount of displacement for smaller blazed cycles is determined by using the slope of the line equation for the linear curve fit of Figure 5.1 that is 4.1 . This means that the displacement due to one cycle is given by $4.1 \times$ Pixel Pitch $=18.04 \mu m$. For example, for $T=0.3$, the displacement is given by 


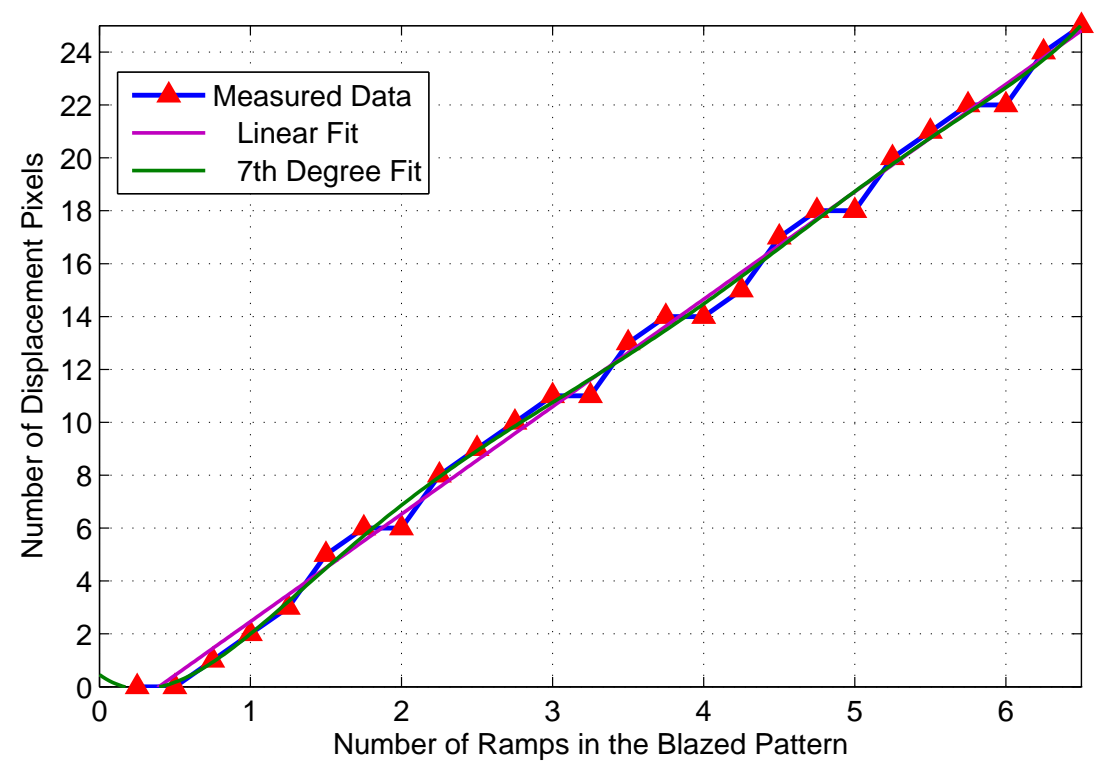

Figure 5.1: Measurement of the number of displacement pixels as a function of the number of ramps in a single blazed pattern. Calculated line equation for the linear fit: $y=4.1 x-1.6$.

$18.04 \mu m \times 0.3=5.412 \mu m$

The magnification of the system is determined by averaging the measured diameter of the image of a few $11 \mu \mathrm{m}$ polystyrene microspheres (Copolymer, P/N: 7510B) with specified size uniformity coefficient of variance of $<18 \%$. With $4.4 \mu m$ pixel pitch of the CCD, the nominal effective magnification of the system is found to be $M=16.4$.

Since two phase ramps are needed to shear the image, the shear amount is twice the displacement distance for a given $T$. In the example of $T=0.3$, the image shear amount is $S_{\text {image }}=10.824 \mu \mathrm{m}$ and the nominal amount of shear in the object plane is given by $S_{\text {object }}=S_{\text {image }} / M=0.660 \mu \mathrm{m}$. 


\subsection{Test Phase Object}

Droplets of highly transmissive microscope immersion oil with refractive index of $n=1.515$ sprayed with a pipette on a microscope slide are used as phase objects. These droplets meet the requirement for a weak phase object because 1) by design they have very minimal effect on the intensity of the light and 2) their formation on the microscope slide has a smooth and slow-varying topography with surface curvature that could be comparable to the wavelength of the light and therefore the light beams exiting them also have slow varying phase lags over their top surface.

Figure 5.2a shows the full field of view of the bright-field image of the droplets. Areas where there are abrupt changes in the intensity that become very large (bright) or very small (dark) indicate a strong phase interaction where there could be strong ray bending and/or crossing present. The indicated droplet in Figure 5.2a has a more uniform intensity distribution compared to other droplets on the slide and hence it is selected as the test droplet. It also has a circular boundary shape which suggests that it could have a dome-like topography. Figure 5.2b shows the cropped view of the bright-field image of this test droplet. With $M=16.4$, the physical dimension of the droplet is approximately $24 \mu m$ in diameter. Although this object does not provide a means for quantitative measurements, it can be used for qualitative analysis and verification of the DIC and PSI operations. 


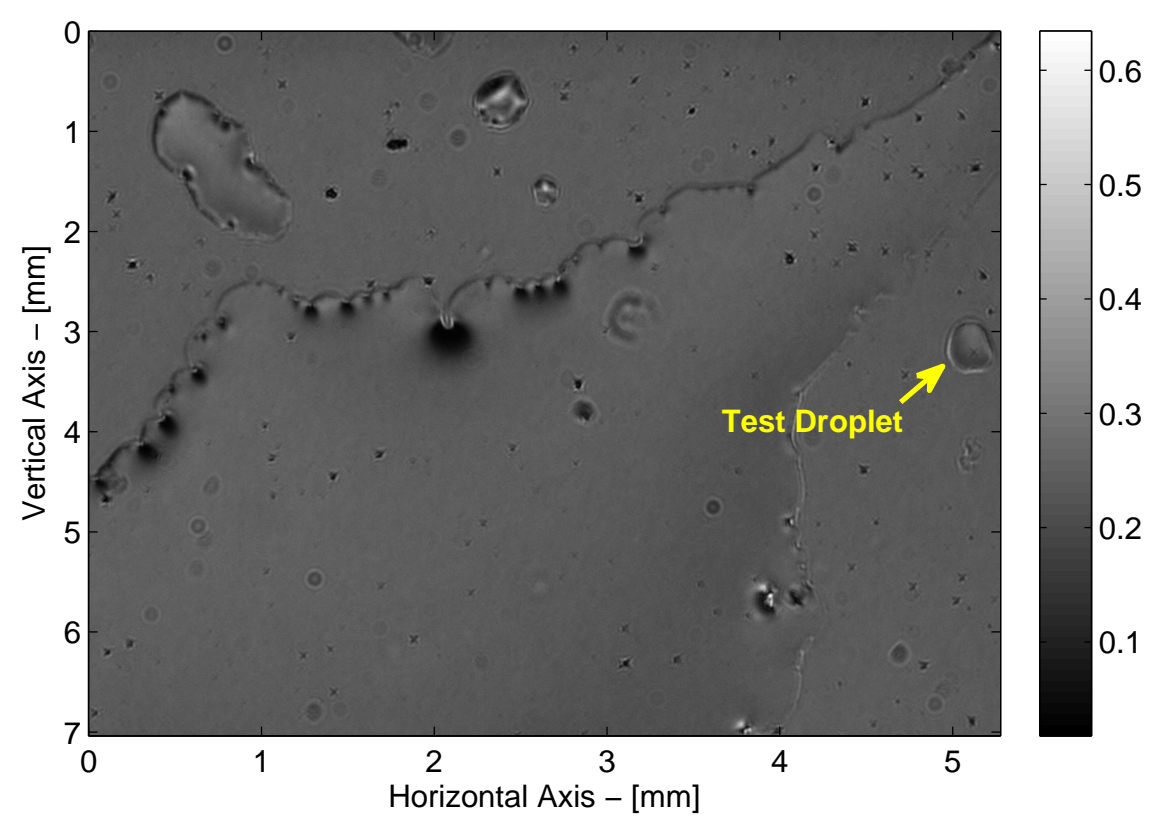

(a) Full field of view of the bright-field image of the immersion oil droplets sprayed on microscope slide with the droplet selected for analysis labeled as 'Test Droplet'.

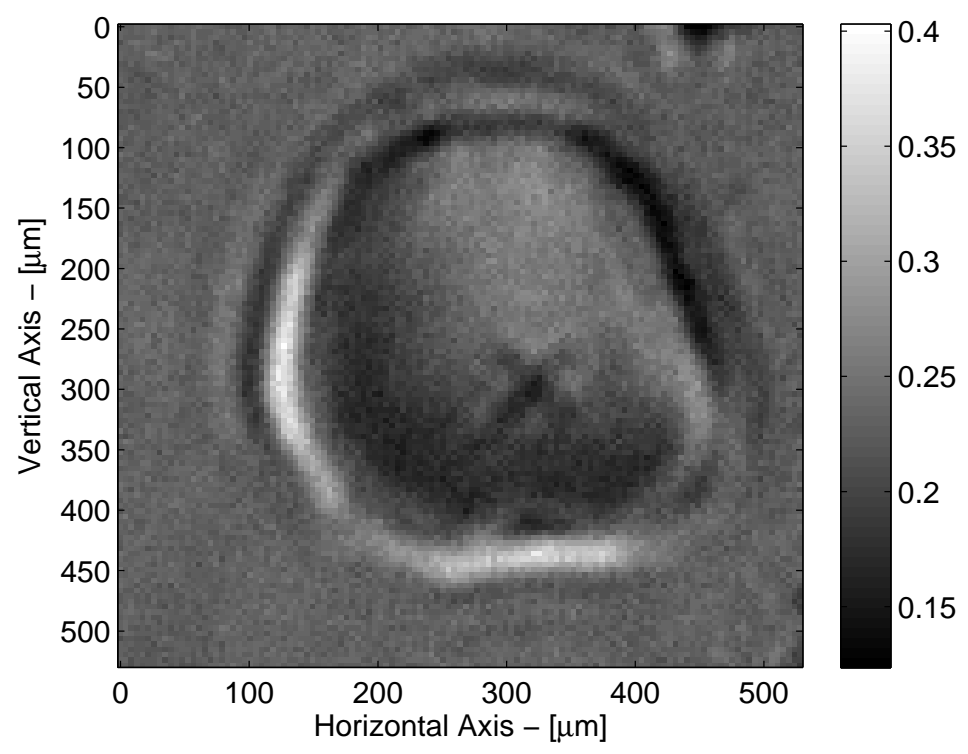

(b) Cropped bright-field image of the test droplet.

Figure 5.2: Grayscale bright-field images of the immersion oil $(n=1.515)$ droplet used as test phase object.

\subsection{Measurement Results}

In this section, a series of measurement results of the test droplet with different shear distances and directions are presented. For a given shear distance and direction, 
the results include the four DIC images $I_{j}$ resulting from every $\theta_{j}$ of PSI (see Chapter 4 for details) and the calculated wrapped phase difference map from these DIC images.

The shear distance is controlled by the number of cycles, $T$, in the blazed pattern and the direction of shear is controlled by the tilt angle of the randomly combined ramp patterns written to the SLM. The number of blazed pattern cycles and their resulting shear distances in the image and object plane that are used in the Figures of this section are listed in Table 5.1.

Table 5.1: Calculated Shear Distance for Number of Cycles in the Blazed Pattern

\begin{tabular}{l|c|c}
$T$ & $S_{\text {image }}[\mu \mathrm{m}]$ & $S_{\text {object }}[\mu \mathrm{m}]$ \\
\hline 0.3 & 10.824 & 0.660 \\
\hline 0.5 & 18.040 & 1.100 \\
\hline 0.75 & 27.060 & 1.650 \\
\hline 1 & 36.080 & 2.200 \\
\hline 1.15 & 41.492 & 2.530 \\
\hline 1.35 & 48.708 & 2.970 \\
\hline 1.45 & 52.316 & 3.190
\end{tabular}

Figures 5.3 through 5.10 show the measurement results with $T=0.3,0.5,1$, and 1.45 for shear directions both along the horizontal and vertical axis respectively. The plots in Figure 5.11a are the cross section of the calculated unwrapped phase difference for $T=0.75,1,1.15$, and 1.35 with shear direction along the horizontal axis. The plots in Figure 5.11b are the cross section of the calculated unwrapped phase difference for $T=0.75,1,1.15$, and 1.35 with shear direction along the vertical axis. A 50-point average of the constant phase offset (background value of $\Delta \Phi$ ) for each of the plots of Figure 5.11 is calculated and subtracted from the values plotted to center them about 0 radians in order to provide a convenient visualization scheme to compare them against each other.

The sizes of the DIC images shown in the following figures are the same as the bright-field image shown in Figure 5.2b. 

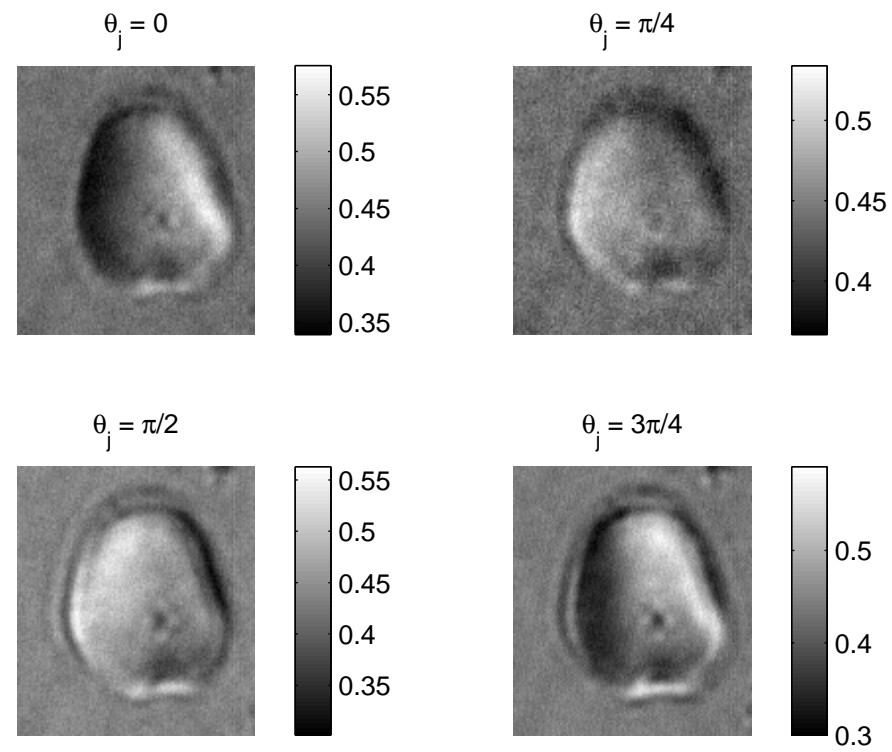

(a) DIC images for each $\theta_{j}$.

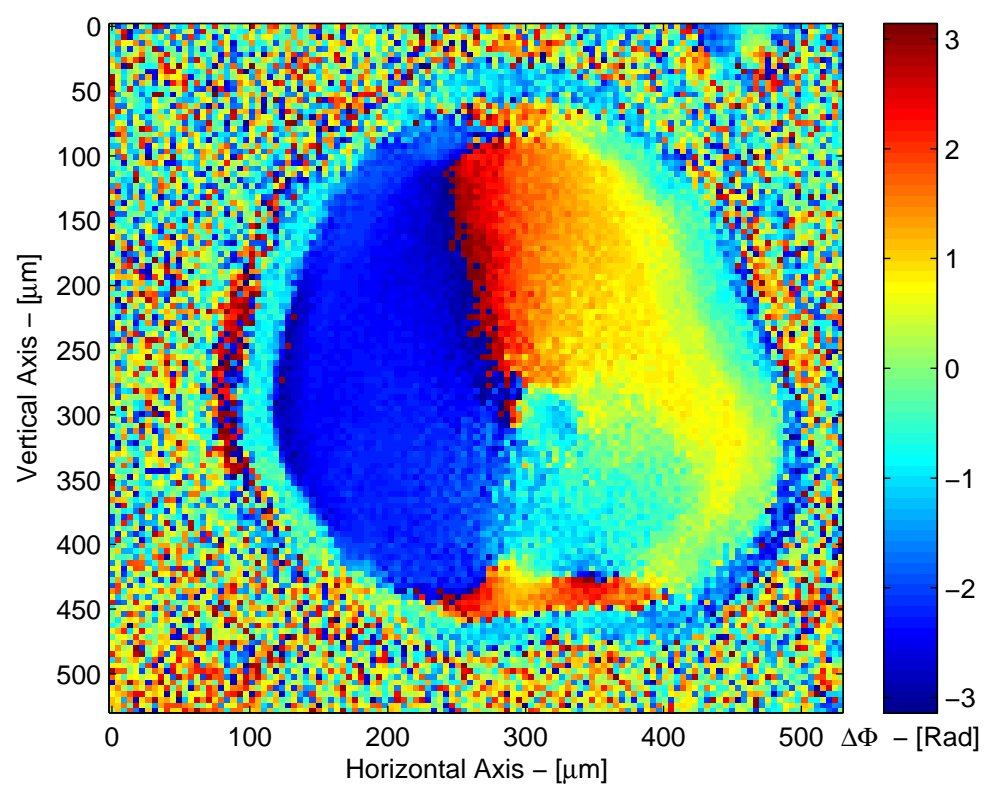

(b) Calculated wrapped phase difference, $\Delta \Phi$.

Figure 5.3: Measurement results of the test droplet with shear direction along the horizontalaxis and $T=0.3\left(S_{\text {image }}=10.824 \mu \mathrm{m}, S_{\text {object }}=0.660 \mu \mathrm{m}\right.$. $)$ 

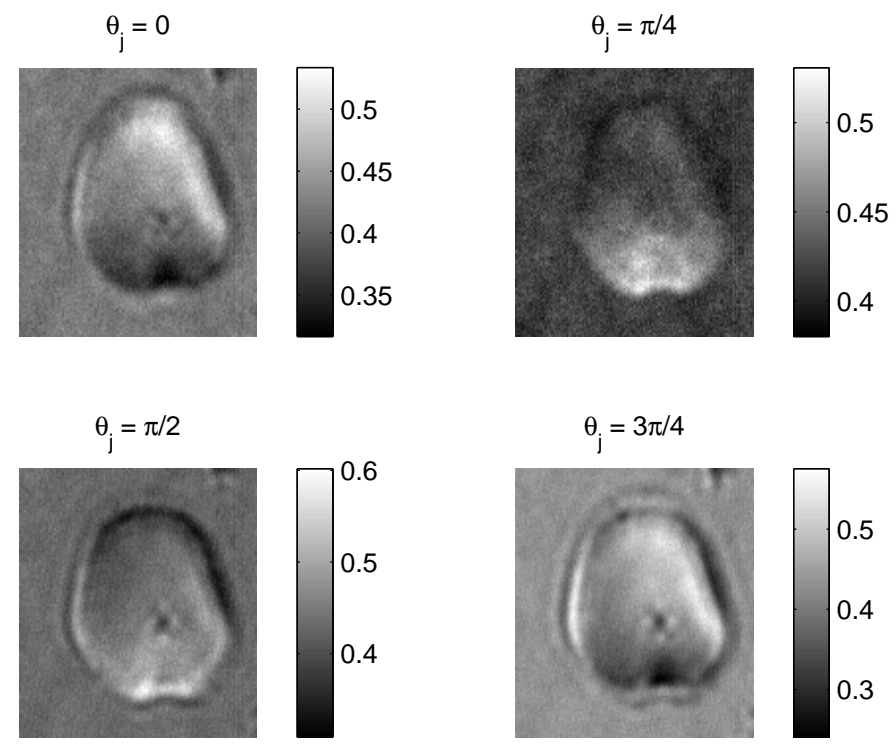

(a) DIC images for each $\theta_{j}$.

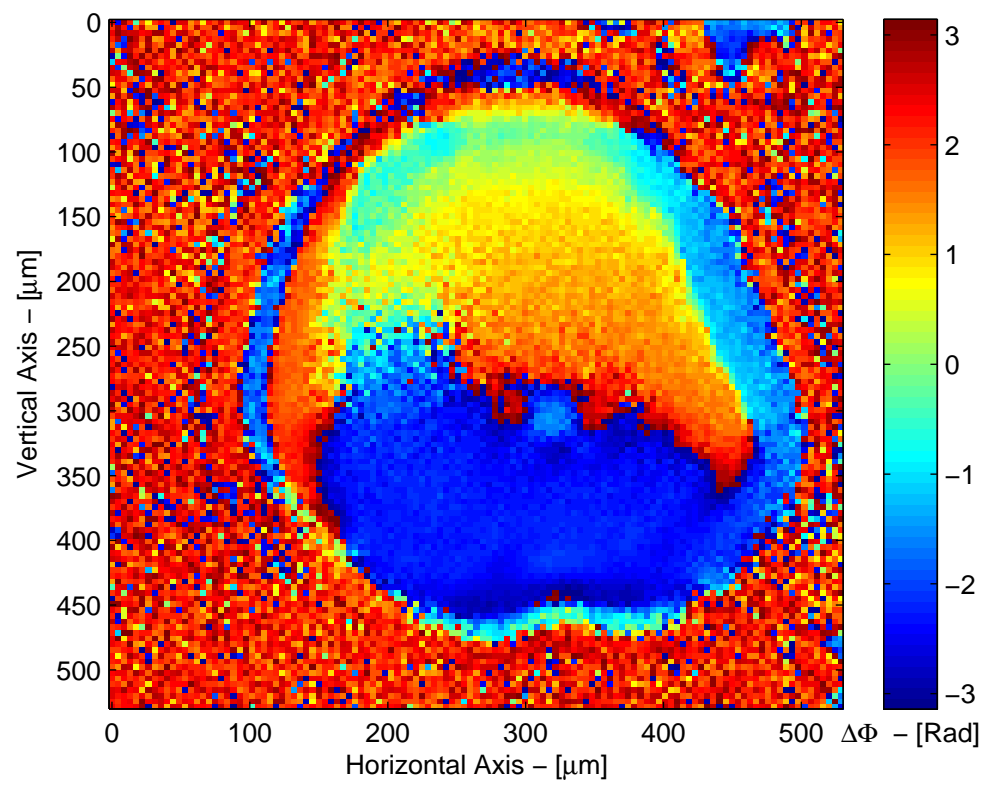

(b) Calculated wrapped phase difference, $\Delta \Phi$.

Figure 5.4: Measurement results of the test droplet with shear direction along the verticalaxis. $T=0.3\left(S_{\text {image }}=10.824 \mu \mathrm{m}, S_{\text {object }}=0.660 \mu \mathrm{m}\right.$. $)$ 

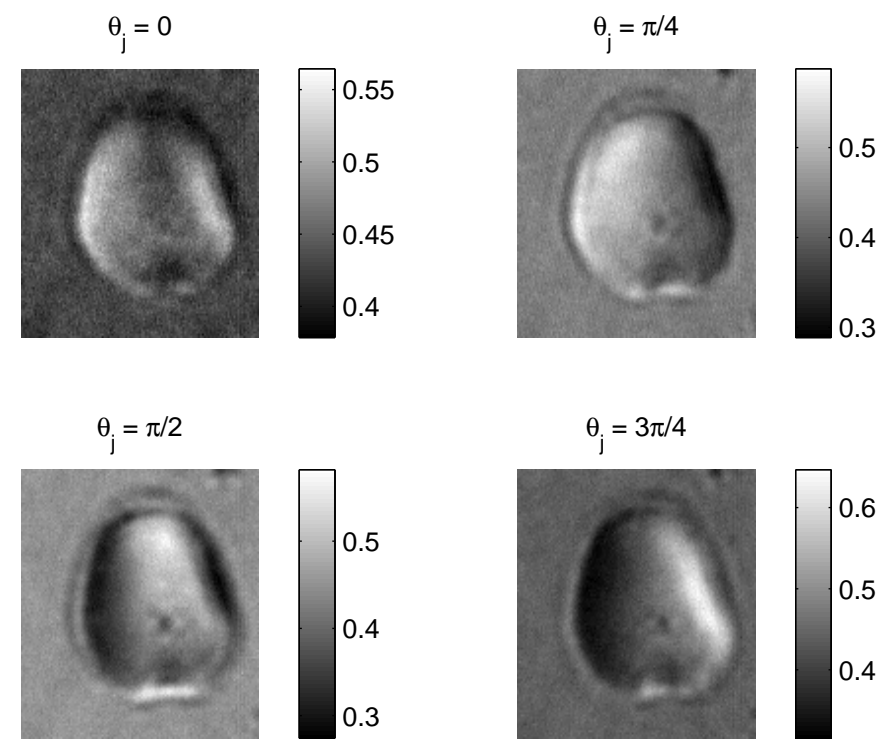

(a) DIC images for each $\theta_{j}$.

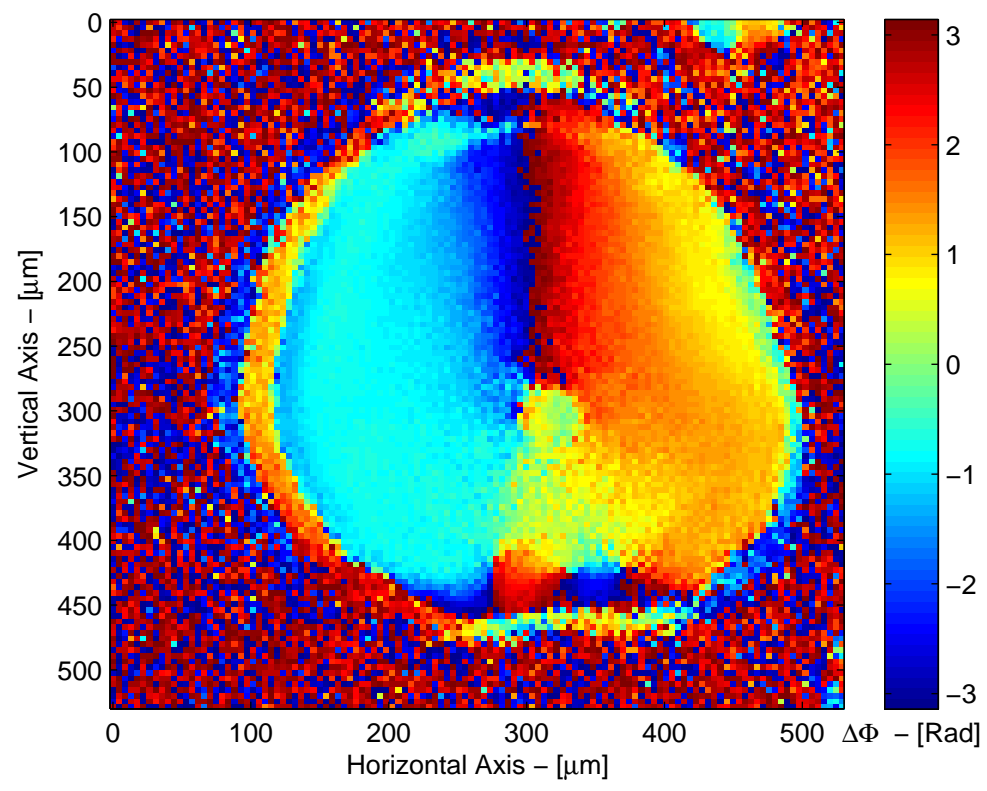

(b) Calculated wrapped phase difference, $\Delta \Phi$.

Figure 5.5: Measurement results of the test droplet with shear direction along the horizontalaxis. $T=0.5\left(S_{\text {image }}=18.040 \mu \mathrm{m}, S_{\text {object }}=1.100 \mu \mathrm{m}\right.$. $)$ 

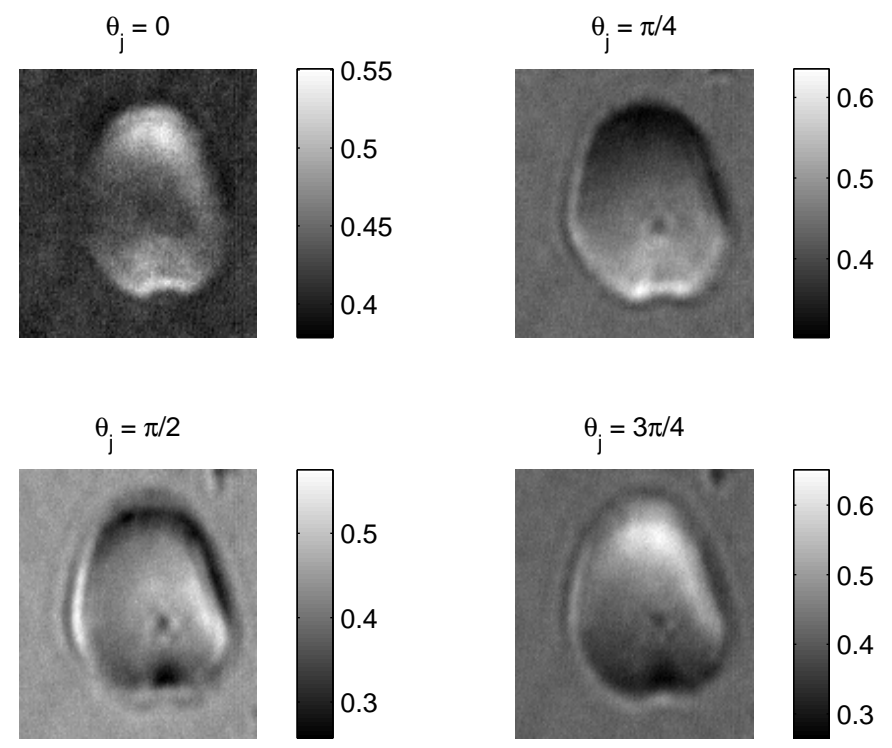

(a) DIC images for each $\theta_{j}$.

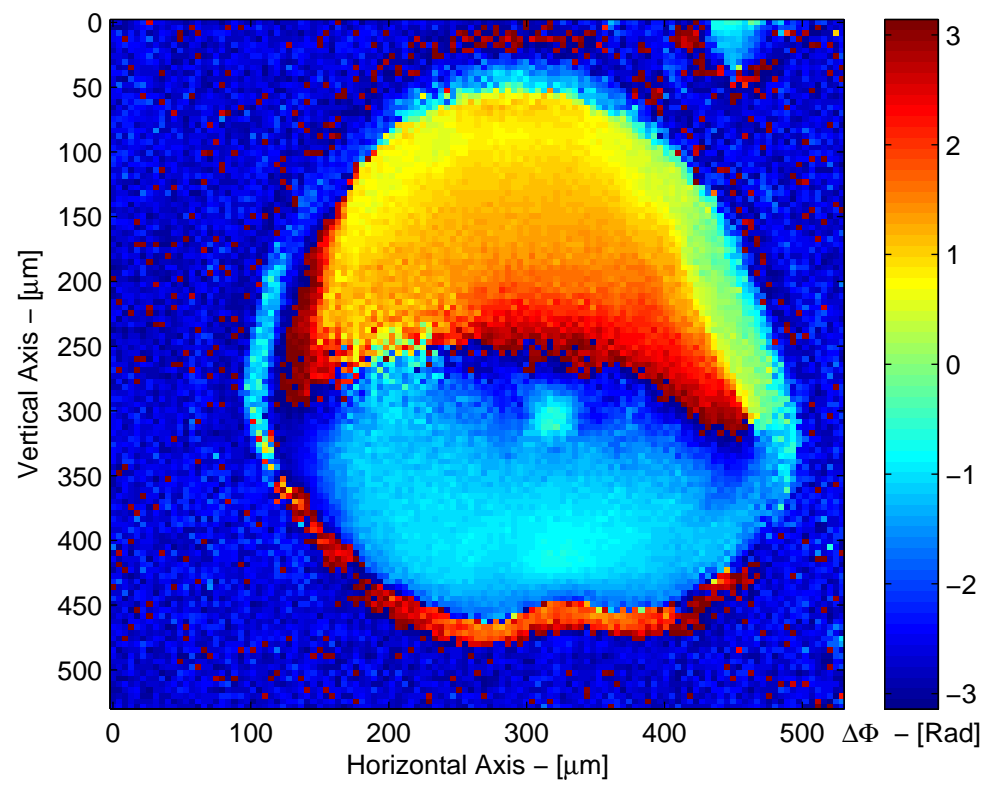

(b) Calculated wrapped phase difference, $\Delta \Phi$.

Figure 5.6: Measurement results of the test droplet with shear direction along the verticalaxis. $T=0.5\left(S_{\text {image }}=18.040 \mu \mathrm{m}, S_{\text {object }}=1.100 \mu \mathrm{m}\right.$. $)$ 

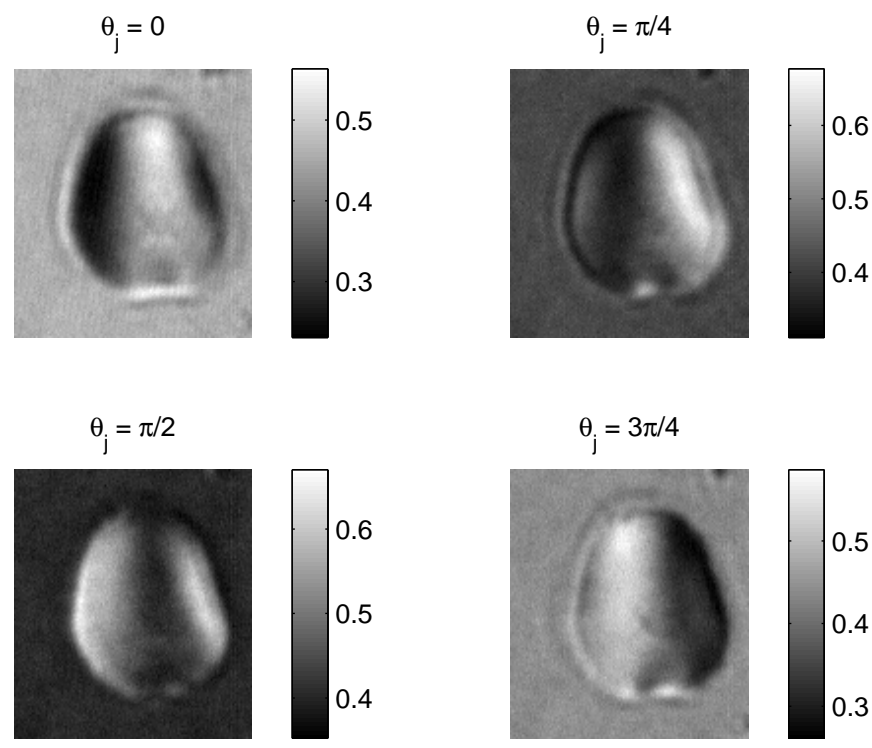

(a) DIC images for each $\theta_{j}$.

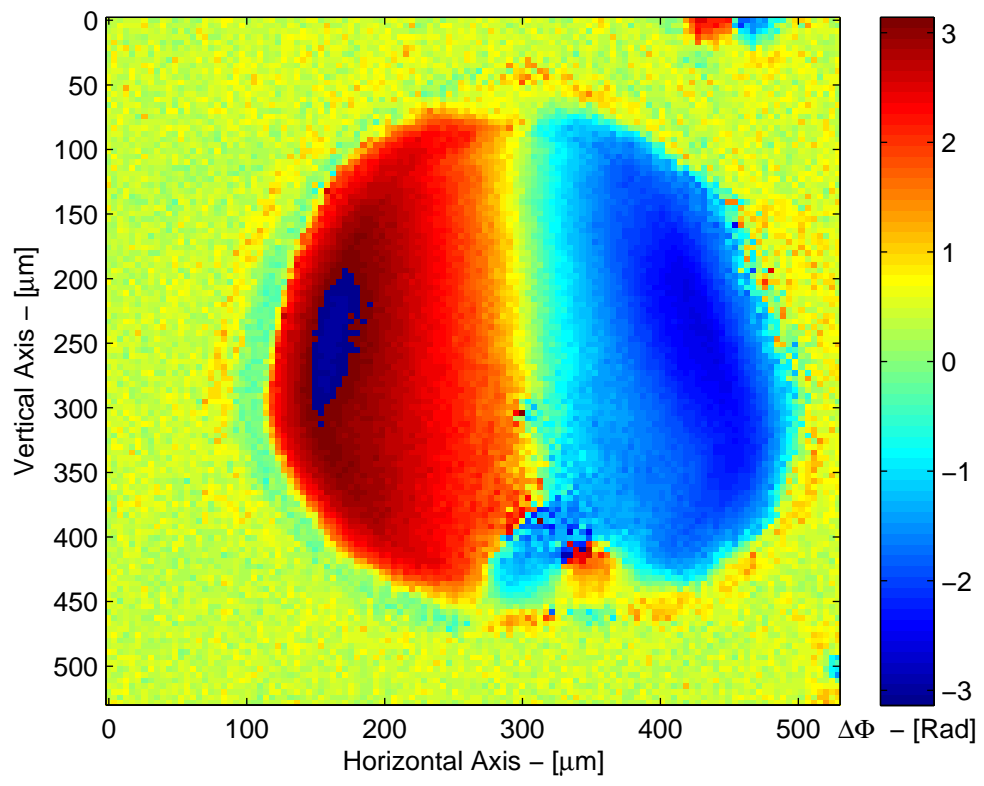

(b) Calculated wrapped phase difference, $\Delta \Phi$.

Figure 5.7: Measurement results of the test droplet with shear direction along the horizontalaxis. $T=1\left(S_{\text {image }}=36.080 \mu \mathrm{m}, S_{\text {object }}=2.200 \mu \mathrm{m}\right.$. $)$ 

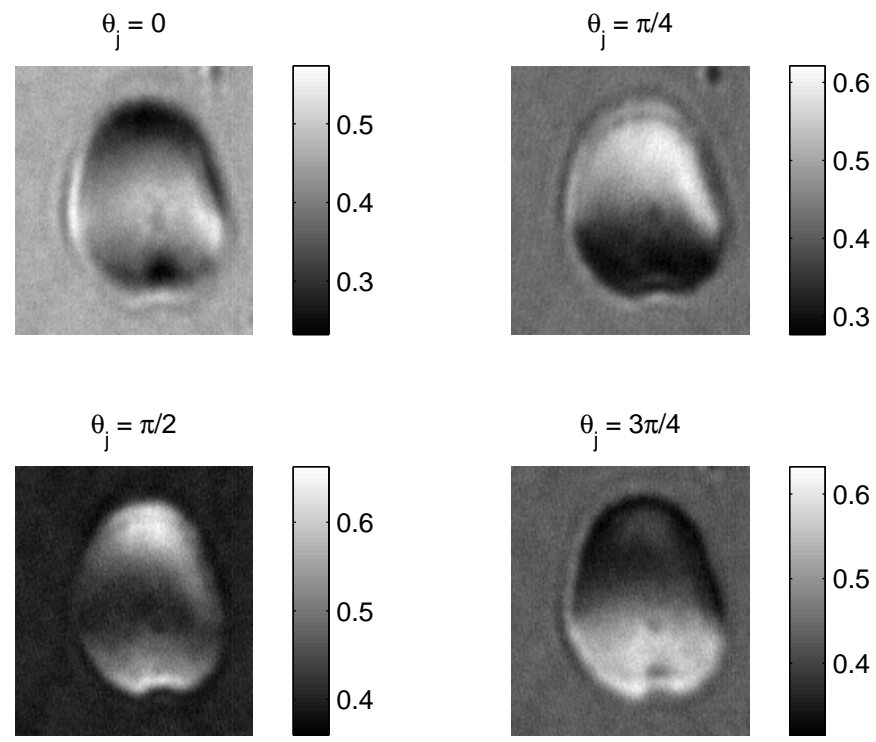

(a) DIC images for each $\theta_{j}$.

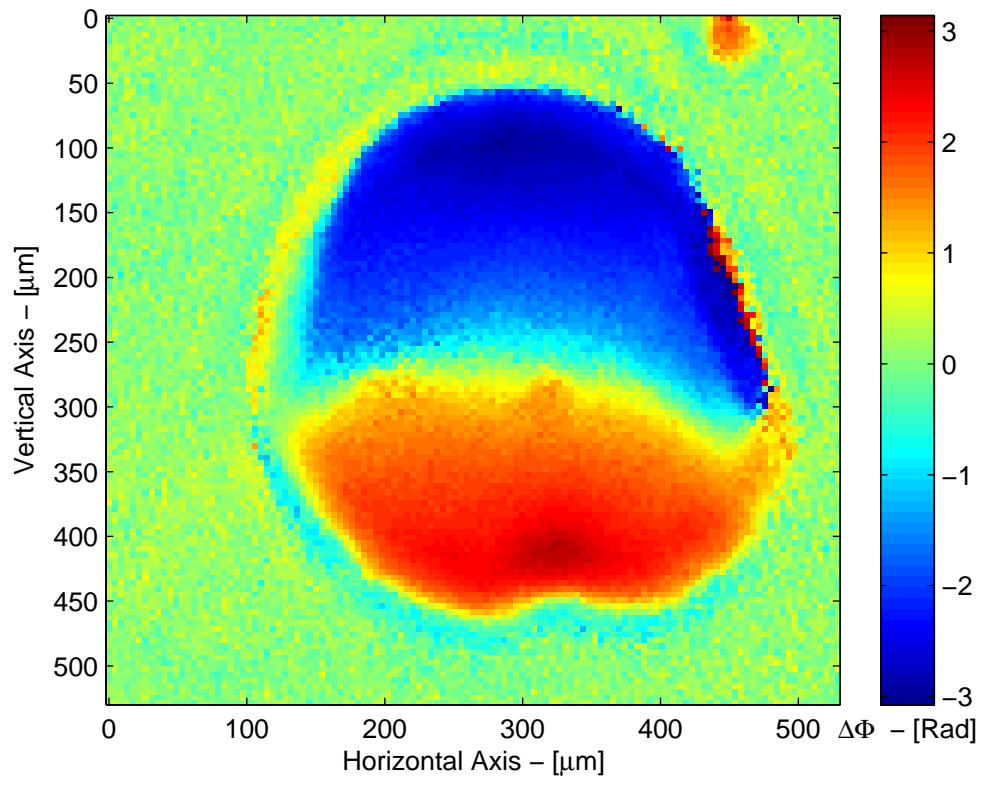

(b) Calculated wrapped phase difference, $\Delta \Phi$.

Figure 5.8: Measurement results of the test droplet with shear direction along the verticalaxis. $T=1\left(S_{\text {image }}=36.080 \mu m, S_{\text {object }}=2.200 \mu \mathrm{m}\right.$. $)$ 

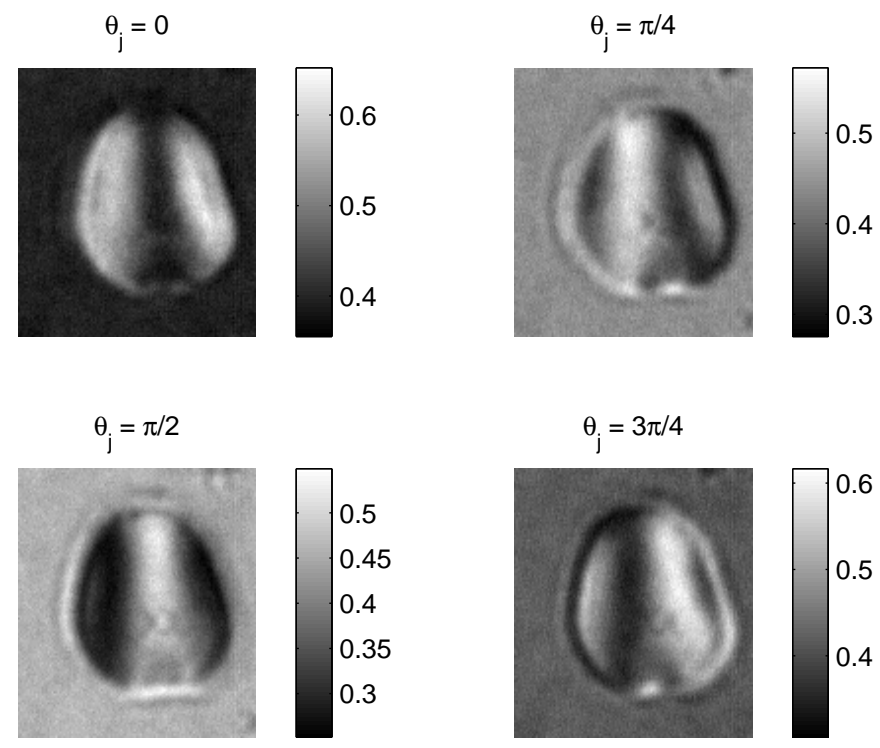

(a) DIC images for each $\theta_{j}$.

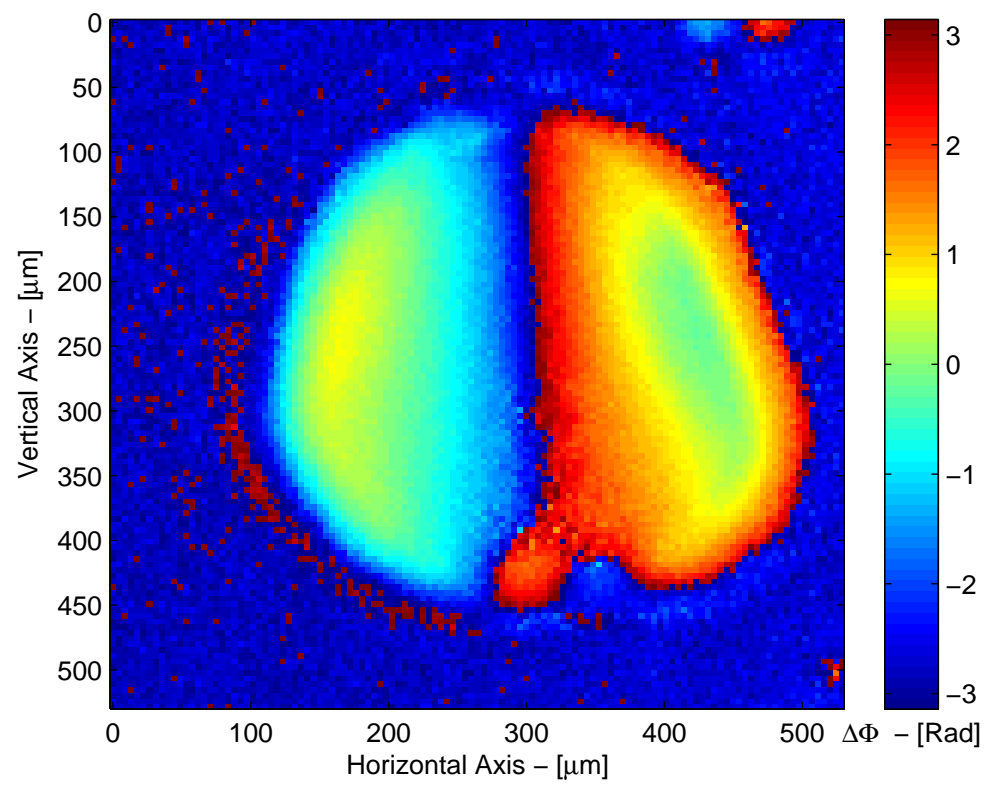

(b) Calculated wrapped phase difference, $\Delta \Phi$.

Figure 5.9: Measurement results of the test droplet with shear direction along the horizontalaxis. $T=1.45\left(S_{\text {image }}=52.316 \mu \mathrm{m}, S_{\text {object }}=3.190 \mu \mathrm{m}\right.$. $)$ 

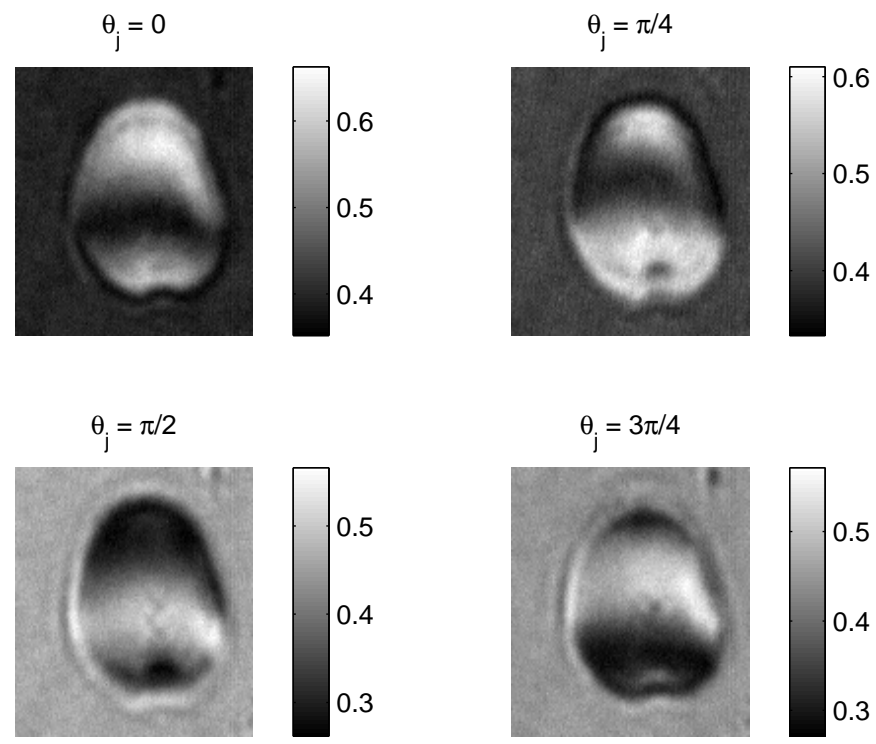

(a) DIC images for each $\theta_{j}$.

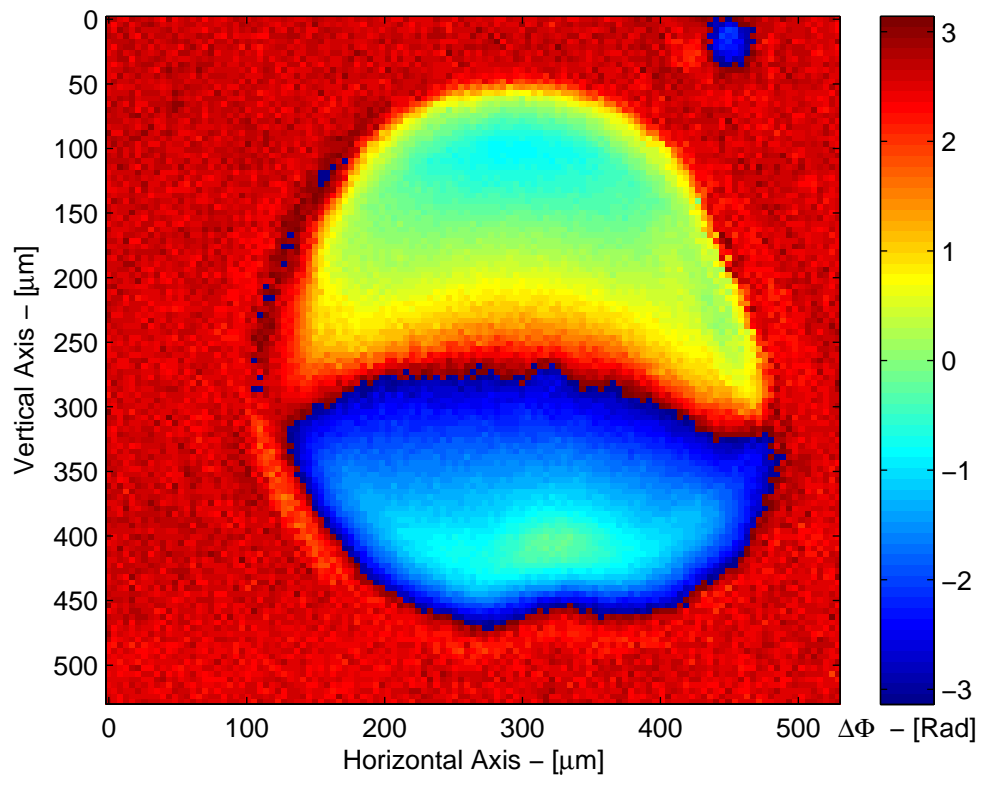

(b) Calculated wrapped phase difference, $\Delta \Phi$.

Figure 5.10: Measurement results of the test droplet with shear direction along the verticalaxis. $T=1.45\left(S_{\text {image }}=52.316 \mu \mathrm{m}, S_{\text {object }}=3.190 \mu \mathrm{m}\right.$. $)$ 


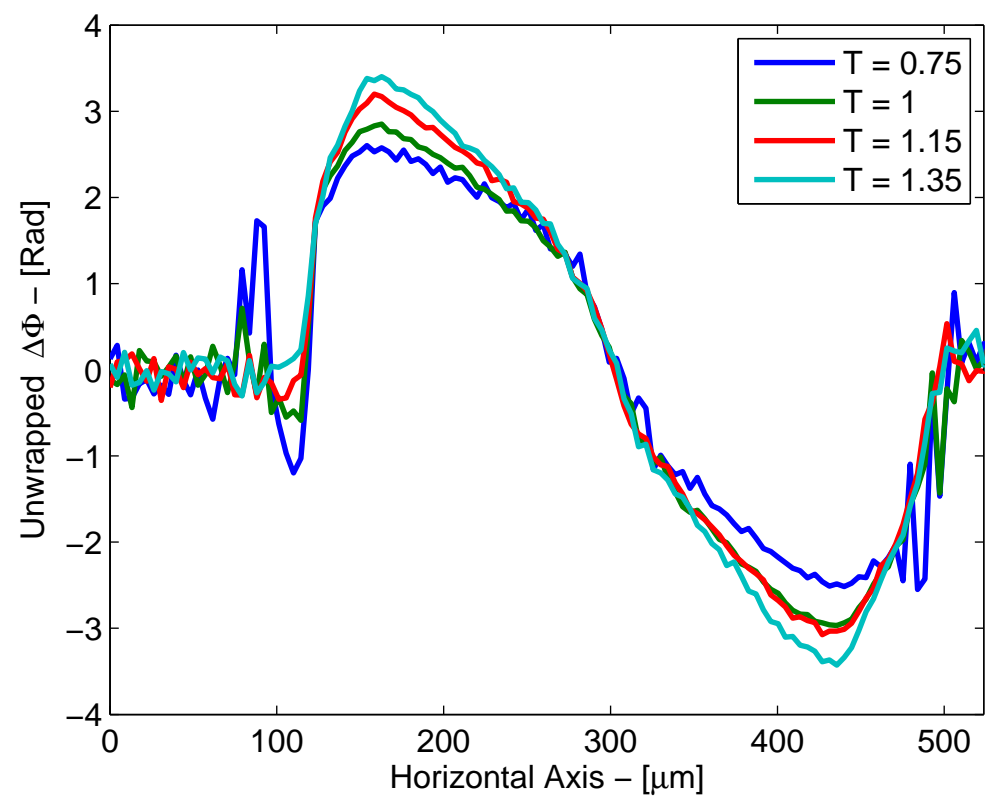

(a) Cross section along the horizontal axis.

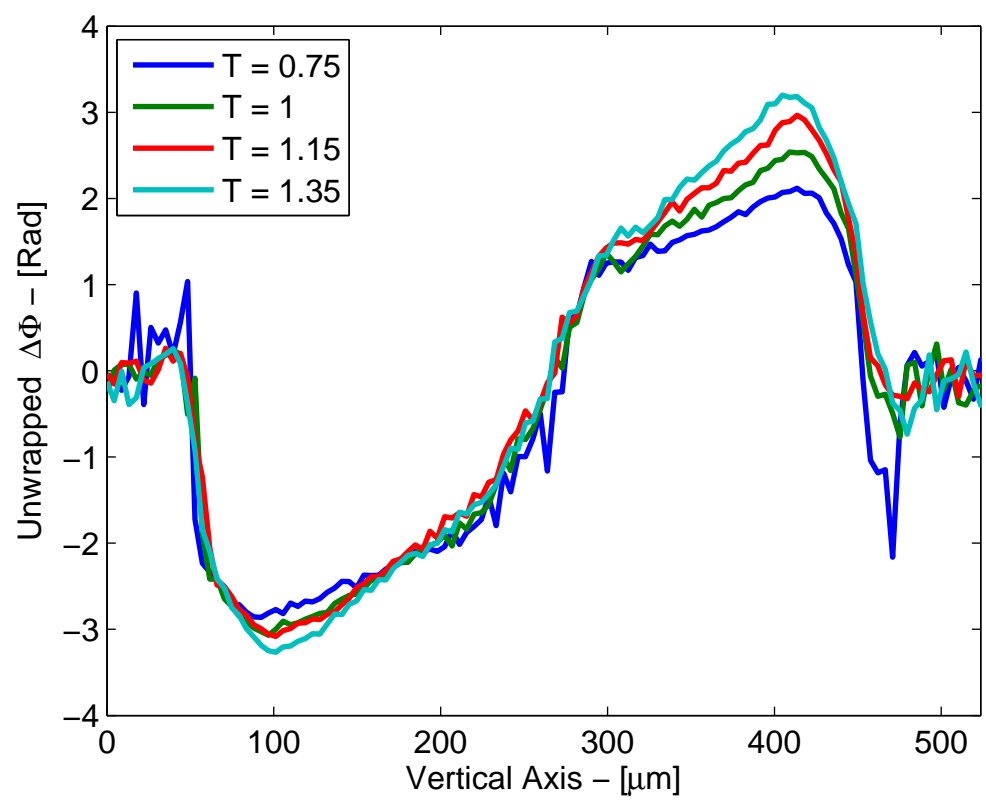

(b) Cross section along the vertical axis.

Figure 5.11: Cross section views of $\Delta \Phi$ with different shear amounts. 


\subsection{Results Analysis}

The discussion in this section aims to validate the DIC operation and object phase difference recovery by means of PSI from the measurement results presented in the previous section.

Qualitatively, the DIC images have all the characteristics of the images obtained by traditional DIC microscopes with Nomarski prism pair where the intensity distribution of the images produce shadow effects that are indicative of the shape of the transparent object along the direction of the shear. Also the objects in the DIC images have enhanced edges where they appear standing out from the background. These features become more pronounced for larger shear amounts. For example, the DIC images for $T=1.45$ in Figures 5.9a and 5.10a reveal the dome-like shape of the droplet as opposed to the brighfield image of the droplet in Figure 5.2b where the droplet appears as a flat-top circular object. The differences in the intensity pattern for every $\theta_{j}$ is also similar to the effects observed with adjusting the bias on a conventional DIC microscope which indicates that the lateral shifting of the SLM's pattern is performing PSI. Additionally, similar characteristics of the DIC images are also demonstrated in the simulation results of Chapter 3. Although these images offer more visual details about the object than the bright-field images, the primary value of them in this work is for calculating the phase difference map.

The numerical simulations in Chapter 3 show that for a semi-spherical weak phase object, $\Delta \Phi$ has an abrupt and large change at the boundaries of the object then it slowly tapers off following the slope of the topography of the object along the shear direction. Over the top of the droplet, $\Delta \Phi$ crosses zero and undergoes sign reversal. The calculated $\Delta \Phi$ maps from measurements also demonstrate such behavior except 
that they are offset by a constant amount (the background value) that is a function of $T$ and the shear direction. $\Delta \Phi$ for $T=1$ shown in Figure $5.8 \mathrm{~b}$ has an offset that is near zero and both the zero crossing along the horizontal border of the apex and sign reversal are simpler to visually observe. The same visual inspection method for other $\Delta \Phi$ maps with larger offset values can be applied by noting that the strip over the top region of the droplet has the same value as the background and considering them as the reference point.

Ideally no phase offset would be present and regardless of the value for $T$ or shear direction all $\Delta \Phi$ maps would be centered at zero. This is based on the assumption that in the frequency plane, the Fourier transform of the object fields is precisely aligned with the SLM pattern along the lateral axis but in practice this is difficult to achieve and the center points of the transformed fields and the SLM may not perfectly coincide. The effect of this misalignment is the introduction of a constant phase error in the pattern's function. Analytically, this misalignment can be modeled by adding an additional term to the pattern's phase offset of Eq. A.6. With this, the expression for $\theta$ becomes:

$$
\theta=\theta_{j}+\theta_{k}+\theta_{0}
$$

where $\theta_{0}$ is the constant offset error. It can be shown that $2 \theta_{0}$ appears as an added term to $\Delta \Phi$ in the argument of the cosine in the intensity expression of Eq. A.10 and since it is a constant, the PSI process cannot discriminate the two. Also this misalignment would yield a different value for $\theta_{0}$ depending on $T$ and the shear direction because this phase offset is the measure of the number of cycles of the blazed pattern that the center points of the transformed fields and the SLM are offset. Nonetheless, $\theta_{0}$ can be readily determined from the background values of the phase difference map and removed. 
The offsets for the cross section plots in Figure 5.11 are removed and it can be seen that their behavior bears a strong resemblance to the simulated cross section plot shown in Figure 3.4b. This verifies the speculation made about the dome-like shape of the droplet. The magnitude of $\Delta \Phi$ is also larger for larger $T$, which validates that the larger values of $T$ produce larger shear amounts and the larger the shear amount the grater is the phase difference. This result is in good agreement with the simulation results for different shear amounts shown in Figure 3.5. This is because for a sloped surface, the phase difference of the beams emerging from two separate test points increases as the separation distance of the test points is increased along the slope.

Another observation regarding the unwrapped plots of Figure 5.11 is that height differences on fractional orders of the illumination laser wavelength $(660 \mathrm{~nm})$ can be measured. The relationship between the height difference, $\Delta h$, and phase difference is given by $\Delta h=\Delta \Phi \lambda / 2 \pi$. For example for $T=0.75$ in Figure $5.11 \mathrm{~b}, \Delta h \approx 25 \mathrm{~nm}$ near the zero crossing point which suggests that over the peak of the droplet the measurement system is capable of detecting height differences as small as nearly $4 \%$ of the wavelength over $1.650 \mu m$ in the object plane. Such small numbers for $\Delta h$ have been reported in the literature where quantitative measurements with verification have been made [40] [38] but those systems have larger than 20x magnifications and are able to achieve smaller shear distances by an order of magnitude. It is for a future work with quantitatively verifiable test objects to evaluate this important aspect of the measurement.

In conclusion, the capability of the system to produce DIC images with adjustable shear amount and direction as well as to perform PSI without any additional components or any mechanical manipulation is verified. Since there is no a priori information 
about the phase object used for the experiments, quantitative analysis is not possible. However, comparing the measurement results obtained with different settings and parameters with simulation results validates the proper operation of the system as a DIC microscope. 
Chapter 6

Conclusion 


\subsection{Summary}

In the initial investigation phase of this work, a number of recent literature concerning modern and novel implementations of phase microscopy techniques using an SLM were surveyed. This provided an overview to bound the objectives and approaches to the research that followed. It also gave an understanding of the state of the art in the field of modern phase microscopy. Reported methods included both the adaptation of traditional phase microscopes such as the Zernike, DIC, and Michaelson and novel approaches such as spiral phase filtering and shearing by exploiting the birefrigerant property of SLM's. It was concluded that compared to other studied methods, shearing interferometry with an SLM requires a simpler process for calibration and characterization for accurate quantitative measurements. Also incorporation of a 4-f system with the SLM as a filter mask in the frequency plane extends the use of the SLM to readily apply PSI for phase information extraction as well. This led to the selection of the DIC technique for the subject of research in the presented work.

Among the publications that were reviewed, of particular interest was [40] in which McIntyre et al. utilized an unconventional way to numerically combine two patterns for a phase-only SLM into one pattern by a randomization process to perform shearing in a DIC microscope system. This inspired an alternative approach from Fourier optics point of view to investigate the motivation for pattern combination in such a manner. To that end, a complete theoretical treatment for explaining the operation of the DIC system using a 4-f system and a phase-only SLM were presented. This also gave way to the development of a novel phase stepping approach that could replace the phase-only SLM with an amplitude-only SLM in the mentioned DIC system.

A set of numerical simulation tools were developed in MATLAB to verify the 
theoretical claims. The simulation cases for the SLM pattern included 1) the ideal DIC where with the traditional 4-step PSI, 2) amplitude cosine transmittance with the 8-step PSI, and 3) randomly combined blazed pattern with both the 4-step and the 8-step PSI. In the first two cases, the simulation results and the theoretical predictions were in agreement. In the case of the randomly combined patterns, the results from the DIC portion of the simulation verified the shearing operation but the DIC images resulting from PSI were cluttered with distortion. Attempts to troubleshoot this by varying the parameters of the simulated system and phase object were unsuccessful. A two-dimensional Gaussian window function with various widths was applied to the SLM function in the frequency domain in order to supress the higher frequency order noise introduced by the randomization. This showed no correlation between the window function and the distortion amount of the DIC images. Since this method has experimentally been applied in this work and in [40] and no such distortion was observed, it is suspected that the distortions are due to numerical artifact. A more thorough numerical analysis and perhaps redesigning of the simulation program for the randomly combined pattern case is left for a future work.

An experimental configuration containing a microscope structure with its output extended to a 4-f system with a phase-only SLM in the frequency plane of the object was built. A custom software controlled both the SLM and retrieved captured images from the CCD. This system implemented a shearing method with an SLM pattern that is a random combination of two phase ramp patterns. The pattern combination process employed a pixel value assignment process using a random binary mask with uniform distribution. Furthermore, PSI was performed without any mechanical adjustments and only by digitally generating a new appropriate pattern for the SLM for every step of the PSI. This system also offered adjustable shear amount and direction through the software. The shear distance of the system as a function of the slope of 
the phase ramp of the SLM pattern was characterized experimentally.

Measurements with different shear amounts and directions were made on droplets of a transmissive immersion oil as test phase objects. The DIC images had the typical shadow effects expected from a traditional Nomarski DIC microscope. These shadow effects increased the contrast of the areas where there was phase variation present. As expected the larger shear amounts resulted in higher contrast in the DIC images of the droplets. The calculated phase gradient maps from the DIC images resulting from the PSI steps also showed expected qualitative characteristics. Comparing the phase difference maps obtained from different shear amounts with earlier simulation results verified the proper operation of the PSI as well.

The implementation of the DIC system for this work differed from the one reported in [40] by McIntyre et al. in two ways. The first difference is that each DIC image measurement step in this work is an average over two sets of measurements: one with the ramp patterns combined with the random binary mask and another one with the patterns combined with the complement of the binary mask. This has the effect of reducing the structured noise introduced by the randomization process. The second difference is that the SLM's phase pattern contained two ramps of equal slopes but opposite signs to perform shearing instead of ramps with different slopes of the same sign. This centers the specular reflection component of the light from the surface of the SLM about the two sheared images and with the help of an additional phase stepping process the effects of the specular reflection can be removed.

In conclusion, an SLM-based closed-loop DIC microscope system with capability to perform quantitative measurements was successfully implemented. The performance of the microscope was qualitatively verified with the aid of the simulation tools developed for this work. Improving the system is possible by increasing the 
magnification and characterizing the system using test objects with known optical and physical properties.

\subsection{Future Work}

Below is a list of improvements and alternative approaches to the current work that the author believes could constitute valuable follow-on projects.

\section{- Troubleshooting the simulation issues for the randomly combined} ramps case. A working simulation process for this case would offer a convenient method to better understand the effect of the randomization process on the point spread function of the system. Also possible optimization for the randomization algorithms can be tested this way. It might be instructional to begin with further expanding the analytical expression of Eq. 2.26 to obtain the point spread function that includes the variable phase term for the PSI $(\theta)$.

- Rebuilding the microscope system with a higher magnification. The current DIC system uses a 10x objective in its imaging path lenses and in combination with L5 (Figure 4.1) it produces an effective magnification of 16.4. For a given blazed pattern number of cycles, larger magnification produces smaller amount of shear. It also provides larger DIC images covering more pixels on the CCD that can result in higher resolution in the calculated phase difference map.

- Using known test phase objects for system characterization and quantitative measurements. This is a necessary step in accurately characterizing two important parameter of the system: 1) the shear amount 2) the smallest resolvable phase difference that can be measured. The use of polystyrene beads 
placed in a closely refractive index matched immersion oil is reported in [37] and [40]. A similar method can be used to create quantifiable weak test phase objects. The use of an arbitrary nanostructure that is also imaged with an electron microscope for reference comparison is another solution.

- Using an amplitude-only SLM instead of a phase-only SLM. Analytically, it was shown that the ramp pattern combination by randomization process is essentially an implementation of the ideal sinusoidal amplitude function. The 8-step PSI presented in Appendix A provides a way to remove the effect of the zeroth order diffraction from a sinusoidal amplitude filter mask in a 4-f system. This was also verified by numerical simulations. Therefore a programmable amplitude-only SLM could replace the phase-only SLM in the DIC system. The effectiveness of the 8-step PSI in removing the effects of the zeroth order in the images can be evaluated using known phase objects in a future work.

- Investigating the use of Carré method for phase information calculations. Initially, the equation for the Carré algorithm from [43] was used to calculate the phase gradient from the phase stepped DIC images. Carré equation offers the advantage that unlike the 4-step method it does not rely on knowing the absolute value of the phase steps and only equal interval between each step is required. However, applying this the results of this work returned a large number of complex values for smaller shear amounts whereas the 4-step algorithm did not have this problem. There are some literature that offer variants of the Carre algorithm to avoid the imaginary number generation. A collection of alternative approaches are available in $[13,44]$ that could be explored for quantitative measurements.

- Implementing the microscope system in reflectance mode configuration. Transmission mode microscopes are easier to build in a bench-top 
arrangement but it consumes a large area. Reflectance mode microscopes are more compact and only require one objective lens for both the illumination and the imaging path which makes them a cheaper alternative. Another advantage is that it allows for changing the objective lens for higher or lower magnifications without readjusting the rest of the components. Phase microscopy in reflectance mode for optically thin objects would result in the amplification of the objects effect on the phase of the incident wavefront due to the double passing of the light through the object. 


\section{Bibliography}

[1] A. Lasslett. Principles and applications of differential interference contrast light microscopy. Microscopy and Analysis, 20:S9-S11, September 2006.

[2] A. Lasslett. Nomarski differential interference-contrast microscopy. part IV. applications. ZEISS Information, 77/78:22-26, 1971.

[3] W. H. Hughes. The advantages and uses of phase contrast microscopy. Journal of Applied Bacteriology, 21(1):143-145, 1958.

[4] Natan Shaked. Biomedical optical phase microscopy and nanoscopy. Academic Press, Amsterdam Boston, 2013.

[5] Fundamental concepts in DIC microscopy. http://www.olympusmicro.com/ primer/techniques/dic/dicintro.html. Accessed: 3/2/2014.

[6] F. Zernike. How I discovered phase contrast. Science, 121(3141):345-349, 1955.

[7] Introduction to phase contrast microscopy. http://www.microscopyu.com/ articles/phasecontrast/phasemicroscopy.html. Accessed: 3/2/2014.

[8] Comparison of phase contrast and DIC microscopy. http://www.microscopyu . com/tutorials/java/phasedicmorph/index.html. Accessed: 3/2/2014.

[9] Etienne Cuche, Frédéric Bevilacqua, and Christian Depeursinge. Digital holog- 
raphy for quantitative phase-contrast imaging. Opt. Lett., 24(5):291-293, Mar 1999.

[10] Christopher Mann, Lingfeng Yu, Chun-Min Lo, and Myung Kim. High-resolution quantitative phase-contrast microscopy by digital holography. Opt. Express, 13(22):8693-8698, Oct 2005.

[11] Michael Totzeck, Norbert Kerwien, Alexander V. Tavrov, Eva Rosenthal, and Hans J. Tiziani. Quantitative Zernike phase-contrast microscopy by use of structured birefringent pupil-filters and phase-shift evaluation, 2002.

[12] Christopher G. Rylander, Digant P. Davé, Taner Akkin, ThomasE. Milner, Kenneth R. Diller, and Ashley J. Welch. Quantitative phase-contrast imaging of cells with phase-sensitive opticalcoherence microscopy. Opt. Lett., 29(13):1509-1511, Jul 2004.

[13] Donald D Duncan, David G Fischer, Amanda Dayton, and Scott A Prahl. Quantitative carré differential interference contrast microscopy to assess phase and amplitude. JOSA A, 28(6):1297-1306, 2011.

[14] Richard W Bowman, Amanda J Wright, and Miles J Padgett. An SLM-based ShackHartmann wavefront sensor for aberration correction in optical tweezers. Journal of Optics, 12(12):124004, 2010.

[15] Kurt D Wulff, Daniel G Cole, Robert L Clark, Roberto DiLeonardo, Jonathan Leach, Jon Cooper, Graham Gibson, and Miles J Padgett. Aberration correction in holographic optical tweezers. Optics express, 14(9):4169-4174, 2006.

[16] Andreas Hermerschmidt, Sven Krüger, Tobias Haist, Susanne Zwick, Michael Warber, and Wolfgang Osten. Holographic optical tweezers with real-time hologram calculation using a phase-only modulating lcos-based SLM at $1064 \mathrm{~nm}$. 
In Integrated Optoelectronic Devices 2008, pages 690508-690508. International Society for Optics and Photonics, 2008.

[17] Vyas Akondi, Sara Castillo, and Brian Vohnsen. Digital pyramid wavefront sensor with tunable modulation. Optics express, 21(15):18261-18272, 2013.

[18] T Shirai, TH Barnes, and TG Haskell. Adaptive wave-front correction by means of all-optical feedback interferometry. Optics letters, 25(11):773-775, 2000.

[19] Quanquan Mu, Zhaoliang Cao, Lifa Hu, Dayu Li, and Li Xuan. An adaptive optics imaging system based on a high-resolution liquid crystal on silicon device. Optics express, 14(18):8013-8018, 2006.

[20] Chao Li, Dayu Li, Quanquan Mu, Li Xuan, and Mingliang Xia. High-resolution retinal imaging through open-loop adaptive optics. Journal of biomedical optics, 15(4):046009-046009, 2010.

[21] Claas Falldorf, Stefan Osten, Christoph V. Kopylow, and Werner Jüptner. Shearing interferometer based on the birefringent properties of a spatial light modulator. Opt. Lett., 34(18):2727-2729, Sep 2009.

[22] C. Falldorf, C. Kopylow, and W. Juptner. Compact lateral shearing interferometer to determine continuous wave fronts. In 3DTV Conference, 200\%, pages 1-4, 2007.

[23] Claas Falldorf. Measuring the complex amplitude of wave fields by means of shear interferometry. J. Opt. Soc. Am. A, 28(8):1636-1647, Aug 2011.

[24] Michael Warber, Susanne Zwick, Malte Hasler, Tobias Haist, and Wolfgang Osten. SLM-based phase-contrast filtering for single and multiple image acquisition. Proc. SPIE, 7442:74420E-74420E-12, 2009. 
[25] Shuai Zhao and Po Sheun Chung. Digital speckle shearing interferometer using a liquid-crystal spatial light modulator. Optical Engineering, 45(10):105606105606, 2006.

[26] Jaeduck Jang, Chae Yun Bae, Je-Kyun Park, and Jong Chul Ye. Self-reference extended depth-of-field quantitative phase microscopy. In BiOS, pages $757018-$ 757018. International Society for Optics and Photonics, 2010.

[27] Jeffrey A. Davis, Dylan E. McNamara, Don M. Cottrell, and Juan Campos. Image processing with the radial Hilbert transform: theory and experiments. Opt. Lett., 25(2):99-101, Jan 2000.

[28] S.N. Khonina, V.V. Kotlyar, M.V. Shinkaryev, V.A. Soifer, and G.V. Uspleniev. The phase rotor filter. Journal of Modern Optics, 39(5):1147-1154, 1992.

[29] N. R. Heckenberg, R. McDuff, C. P. Smith, and A. G. White. Generation of optical phase singularities by computer-generated holograms. Opt. Lett., 17(3):221223, Feb 1992.

[30] Alipasha Vaziri, Gregor Weihs, and Anton Zeilinger. Superpositions of the orbital angular momentum for applications in quantum experiments. Journal of Optics B: Quantum and Semiclassical Optics, 4(2):S47, 2002.

[31] Severin Fürhapter, Alexander Jesacher, Stefan Bernet, and Monika RitschMarte. Spiral phase contrast imaging in microscopy. Opt. Express, 13(3):689-694, Feb 2005.

[32] Severin Fürhapter, Alexander Jesacher, Stefan Bernet, and Monika RitschMarte. Spiral interferometry. Opt. Lett., 30(15):1953-1955, Aug 2005. 
[33] Alexander Jesacher, Severin Fürhapter, Stefan Bernet, and Monika RitschMarte. Shadow effects in spiral phase contrast microscopy. Phys. Rev. Lett., 94:233902, Jun 2005.

[34] Alexander Jesacher, Severin Fürhapter, Stefan Bernet, and Monika RitschMarte. Spiral interferogram analysis. J. Opt. Soc. Am. A, 23(6):1400-1409, Jun 2006.

[35] Stefan Bernet, Alexander Jesacher, Severin Fürhapter, Christian Maurer, and Monika Ritsch-Marte. Quantitative imaging of complex samples by spiral phase contrast microscopy. Opt. Express, 14(9):3792-3805, May 2006.

[36] A. Jesacher, A. Schwaighofer, S. Fürhapter, C. Maurer, S. Bernet, and M. RitschMarte. Wavefront correction of spatial light modulators using an optical vortex image. Opt. Express, 15(9):5801-5808, Apr 2007.

[37] Christian Maurer, Stefan Bernet, and Monika Ritsch-Marte. Refining common path interferometry with a spiral phase Fourier filter. Journal of Optics A: Pure and Applied Optics, 11(9):094023, 2009.

[38] Christian Maurer, Stefan Bernet, and Monika Ritsch-Marte. Spiral Phase Contrast Microscopy, pages 143-154. Wiley-VCH Verlag GmbH and Co. KGaA, 2011.

[39] Timothy J. McIntyre, Christian Maurer, Stefan Bernet, and Monika RitschMarte. Differential interference contrast imaging using a spatial light modulator. Opt. Lett., 34(19):2988-2990, Oct 2009.

[40] Timothy J. McIntyre, Christian Maurer, Stephanie Fassl, Saranjam Khan, Stefan Bernet, and Monika Ritsch-Marte. Quantitative SLM-based differential interference contrast imaging. Opt. Express, 18(13):14063-14078, Jun 2010. 
[41] Jeffrey A. Davis and Don M. Cottrell. Random mask encoding of multiplexed phase-only and binary phase-only filters. Opt. Lett., 19(7):496-498, Apr 1994.

[42] Horst Schreiber and John H. Bruning. Optical Shop Testing, chapter 14. Wiley, third edition, 2007.

[43] Gary Cloud. Optical Methods of Engineering Analysis, chapter 22. Cambridge University Press, 1998.

[44] Manuel Servin, J Antonio Quiroga, and Moises Padilla. Fringe Pattern Analysis for Optical Metrology: Theory, Algorithms, and Applications. John Wiley \& Sons, 2014. 


\section{Appendix A}

\section{Sinusoidal Amplitude Filtering}

The general function of an amplitude cosine filter in the frequency plane of a $4-\mathrm{f}$ modulation system is given by:

$$
\begin{aligned}
H_{S L M}\left(f_{x}, f_{y}\right) & =\frac{1}{2}+\frac{1}{2} \cos \left[2 \pi\left(S_{x} f_{x}+S_{y} f_{y}\right)+\theta\right] \\
& =\frac{1}{2}+\frac{1}{4}\left[e^{i\left[2 \pi\left(S_{x} f_{x}+S_{y} f_{y}\right)+\theta\right]}+e^{-i\left[2 \pi\left(S_{x} f_{x}+S_{y} f_{y}\right)+\theta\right]}\right]
\end{aligned}
$$

Where $S_{x}$ and $S_{y}$ are the filter pattern's spatial frequencies along the x and y-axis respectively and $\theta$ is the phase offset that is assumed to be adjustable and used for PSI. The objective is to derive the intensity expression at the detector plane with the object fields given by $A(x, y) e^{i \Phi(x, y)}$ at the input of the 4-f modulation system. For the sake of simplicity in the example that follows, the cosine pattern only has a frequency component along the x-axis (e.g. $S_{y}=0$ ) and $s$ is used as the pattern frequency (e.g. $S_{x}=s$ ). With this, the filter's function becomes:

$$
\begin{aligned}
H_{S L M}\left(f_{x}, f_{y}\right) & =\frac{1}{2}+\frac{1}{2} \cos \left[2 \pi s f_{x}+\theta\right] \\
& =\frac{1}{2}+\frac{1}{4}\left[e^{i\left(2 \pi s f_{x}+\theta\right)}+e^{-i\left(2 \pi s f_{x}+\theta\right)}\right]
\end{aligned}
$$


Applying the filter to the Fourier transform of the object fields forms the following transform pair:

$$
\begin{aligned}
& H_{S L M}\left(f_{x}, f_{y}\right) \mathcal{F}\left\{A(x, y) e^{i \Phi(x, y)}\right\}\left(f_{x}, f_{y}\right) \Longleftrightarrow \\
& \frac{1}{2} A(x, y) e^{i \Phi(x, y)}+\frac{1}{4} A(x+s, y) e^{i[\Phi(x+s, y)+\theta]}+\frac{1}{4} A(x-s, y) e^{i[\Phi((x-s, y)-\theta]}
\end{aligned}
$$

For a phase object, $A(x, y)$ is assumed to be very small which means that the object mainly affects the phase fields passing through it. For completeness, the amplitude factor of the object will be accounted for henceforth. The expression for the intensity is given by:

$$
\begin{aligned}
I(x, y)= & {\left[\frac{1}{2} A(x, y) e^{i \Phi(x, y)}+\frac{1}{4} A(x+s, y) e^{i[\Phi(x+s, y)+\theta]}+\frac{1}{4} A(x-s, y) e^{i[\Phi(x-s, y)-\theta]}\right] \times } \\
& {\left[\frac{1}{2} A(x, y) e^{-i \Phi(x, y)}+\frac{1}{4} A(x+s, y) e^{-i[\Phi(x+s, y)+\theta]}+\frac{1}{4} A(x-s, y) e^{-i[\Phi(x-s, y)-\theta]}\right] } \\
= & \frac{1}{4} A(x, y)^{2}+\frac{1}{16} A(x+s, y)^{2}+\frac{1}{16} A(x-s, y)^{2}+ \\
& \frac{1}{8} A(x, y) A(x+s, y)\left[e^{i[\Phi(x+s, y)-\Phi(x, y)+\theta]}+e^{-i[\Phi(x+s, y)-\Phi(x, y)+\theta]}\right]+ \\
& \frac{1}{8} A(x, y) A(x-s, y)\left[e^{i[\Phi(x, y)-\Phi(x-s, y)+\theta]}+e^{-i[\Phi(x, y)-\Phi(x-s, y)+\theta]}\right]+ \\
& \frac{1}{16} A(x+s, y) A(x-s, y)\left[e^{i[\Phi(x+s, y)-\Phi(x-s, y)+2 \theta]}+e^{-i[\Phi(x+s, y)-\Phi(x-s, y)+2 \theta]}\right] \text { (A.4) }
\end{aligned}
$$

Using the Euler's identity, this simplifies to:

$$
\begin{aligned}
I(x, y)= & \frac{1}{4} A(x, y)^{2}+\frac{1}{16} A(x+s, y)^{2}+\frac{1}{16} A(x-s, y)^{2}+ \\
& \frac{1}{4} A(x, y) A(x+s, y) \cos [\Phi(x+s, y)-\Phi(x, y)+\theta]+ \\
& \frac{1}{4} A(x, y) A(x-s, y) \cos [\Phi(x, y)-\Phi(x-s, y)+\theta]+ \\
& \frac{1}{8} A(x+s, y) A(x-s, y) \cos [\Phi(x+s, y)-\Phi(x-s, y)+2 \theta]
\end{aligned}
$$


Where the first three terms are the constant DC terms and, unlike the ideal DIC case, there are three cosine terms. Two contain the "half-shear" terms $\Phi(x+s, y)-\Phi(x, y)$ and $\Phi(x, y)-\Phi(x-s, y)$ that are are the results of the interference of the zeroth order diffraction component with the +1 and -1 diffraction orders of the amplitude sinusoidal pattern and one carries the familiar phase difference $\Delta \Phi(x, y)=\Phi(x+$ $s, y)-\Phi(x-s, y)$. An interesting and useful result is that the phase offset of the pattern appears with a factor of 2 in the phase difference cosine term whereas the half-shear terms preserve the pattern's phase offset value.

Extracting $\Delta \Phi$ from Eq. A.5 by PSI discussed in section 2.6 is not possible because the cosine terms carrying the half-shear information are also inseparable parts of the intensity expression but they can be eliminated with the aid of an additional phase stepping process. To do so, $\theta$ is divided in two parts:

$$
\theta=\theta_{j}+\theta_{k}
$$

Where $\theta_{j}$ is the variable phase term for PSI, and

$$
\theta_{k}= \begin{cases}0 & k=0 \\ \pi & k=1\end{cases}
$$

With this, the intensity expression of Eq. A.5 becomes:

$$
\begin{aligned}
I_{j k}(x, y)= & \frac{1}{4} A(x, y)^{2}+\frac{1}{16} A(x+s, y)^{2}+\frac{1}{16} A(x-s, y)^{2}+ \\
& \frac{1}{4} A(x, y) A(x+s, y) \cos \left[\Phi(x+s, y)-\Phi(x, y)+\theta_{j}+\theta_{k}\right]+ \\
& \frac{1}{4} A(x, y) A(x-s, y) \cos \left[\Phi(x, y)-\Phi(x-s, y)+\theta_{j}+\theta_{k}\right]+ \\
& \left.\frac{1}{8} A(x+s, y) A(x-s, y) \cos \left[\Delta \Phi(x, y)+2 \theta_{j}+2 \theta_{k}\right)\right]
\end{aligned}
$$


Using the trigonometry identity $\cos (x)=-\cos (x+\pi)$ :

$$
\begin{aligned}
I_{j 0}(x, y)+I(x, y)_{j 1}= & \frac{1}{2} A(x, y)^{2}+\frac{1}{8} A(x+s, y)^{2}+\frac{1}{8} A(x-s, y)^{2}+ \\
& \frac{1}{4} A(x+s, y) A(x-s, y) \cos \left[\Delta \Phi(x, y)+2 \theta_{j}\right]
\end{aligned}
$$

Making the following substitutions:

$$
\begin{aligned}
\alpha & =\frac{1}{2} A(x, y)^{2}+\frac{1}{8} A(x+s, y)^{2}+\frac{1}{8} A(x-s, y)^{2} \\
\beta & =\frac{1}{4} A(x+s, y) A(x-s, y)
\end{aligned}
$$

gives:

$$
I(x, y)_{j}=\alpha+\beta \cos \left[\Delta \Phi(x, y)+2 \theta_{j}\right]
$$

which is the well-known interference formula for intensity given by Eq. 2.8 that phase-shifting interferometry can be applied to in order to extract the argument of the cosine term. 


\section{Appendix B}

\section{Analytical Analysis of Shearing with Randomly Multiplexed Phase Ramp Filter Functions in a 4-f System}

Starting with the SLM function containing two randomly multiplexed ramps of opposite slopes along the $f_{x}$ direction:

$$
\begin{aligned}
H_{S L M}\left(f_{x}, f_{y}\right) & =e^{R i\left(2 \pi s f_{x}+\theta\right)-\bar{R} i\left(2 \pi s f_{x}+\theta\right)} \\
& =R e^{i\left(2 \pi s f_{x}+\theta\right)}+\bar{R} e^{-i\left(2 \pi s f_{x}+\theta\right)}
\end{aligned}
$$

where $R$ is a two dimensional random binary (with values of 0 or 1 ) function in the $f_{x}, f_{y}$ plane and $\bar{R}$ is the complement of $R$. This allows to write the SLM function in the form of a summation of two exponential terms. For example when $R\left(f_{x}, f_{y}\right)=1$ :

$$
\begin{aligned}
H_{S L M}\left(f_{x}, f_{y}\right) & =e^{i\left(2 \pi s f_{x}+\theta\right)-0} \\
& =e^{i\left(2 \pi s f_{x}+\theta\right)}+0
\end{aligned}
$$

and when $R\left(f_{x}, f_{y}\right)=0$ :

$$
\begin{aligned}
H_{S L M}\left(f_{x}, f_{y}\right) & =e^{0-i\left(2 \pi s f_{x}+\theta\right)} \\
& =0+e^{-i\left(2 \pi s f_{x}+\theta\right)}
\end{aligned}
$$


The output fields are given by:

$$
\begin{aligned}
E_{\text {out }}(x, y)= & \mathcal{F}\left\{\mathcal{F}\left\{A(x, y) e^{i \Phi(x, y)}\right\} H_{S L M}\right\} \\
= & \mathcal{F}\left\{\mathcal{F}\left\{A(x, y) e^{i \Phi(x, y)}\right\}\right\} * \mathcal{F}\left\{R e^{i\left(2 \pi s f_{x}+\theta\right)}+\bar{R} e^{-i\left(2 \pi s f_{x}+\theta\right)}\right\} \\
= & A(-x,-y) e^{i \Phi(-x,-y)} *\left[\mathcal{F}\left\{R e^{i\left(2 \pi s f_{x}+\theta\right)}\right\}+\mathcal{F}\left\{\bar{R} e^{-i\left(2 \pi s f_{x}+\theta\right)}\right\}\right] \\
= & A(-x,-y) e^{i \Phi(-x,-y)} * \mathcal{F}\{R\} * \mathcal{F}\left\{e^{i\left(2 \pi s f_{x}+\theta\right)}\right\} \\
& \quad+A(-x,-y) e^{i \Phi(-x,-y)} * \mathcal{F}\{\bar{R}\} * \mathcal{F}\left\{e^{-i\left(2 \pi s f_{x}+\theta\right)}\right\} \\
= & \mathcal{F}\{R\} * A(-x+s,-y) e^{i[\Phi(-x+s,-y)+\theta]} \\
& \quad+\mathcal{F}\{\bar{R}\} * A(-x-s,-y) e^{-i[\Phi(-x-s,-y)-\theta]}
\end{aligned}
$$

Making the following substitutions:

$$
\begin{aligned}
\Gamma & \equiv \mathcal{F}\{R\} \\
\bar{\Gamma} & \equiv \mathcal{F}\{\bar{R}\} \\
\Phi^{+} & \equiv \Phi(-x+s,-y) \\
\Phi^{-} & \equiv \Phi(-x-s,-y) \\
A^{+} & \equiv A(-x+s,-y) \\
A^{-} & \equiv A(-x-s,-y) \\
\Delta \Phi & \equiv \Phi^{+}-\Phi^{-}
\end{aligned}
$$

the expression for intensity becomes:

$$
\begin{aligned}
I= & E_{\text {out }} E_{\text {out }}^{*} \\
=[ & {\left[\Gamma * A^{+} e^{i\left(\Phi^{+}+\theta\right)}+\bar{\Gamma} * A^{-} e^{i\left(\Phi^{-}-\theta\right)}\right] } \\
& \times\left[\Gamma^{*} * A^{+} e^{-i\left(\Phi^{+}+\theta\right)}+\bar{\Gamma}^{*} * A^{-} e^{-i\left(\Phi^{-}-\theta\right)}\right]
\end{aligned}
$$


that simplifies to:

$$
\begin{array}{rl}
I=A^{+^{2}} & * \Gamma * \Gamma^{*}+A^{-^{2}} * \bar{\Gamma} * \bar{\Gamma}^{*} \\
& +A^{+} A^{-}\left(\Gamma * \bar{\Gamma}^{*}\right) * e^{i(\Delta \Phi+2 \theta)}+A^{+} A^{-}\left(\Gamma^{*} * \bar{\Gamma}\right) * e^{-i(\Delta \Phi+2 \theta)}
\end{array}
$$

This can be re-written as:

$$
\begin{aligned}
I=\left(\Gamma * \Gamma^{*}\right) * A^{+^{2}}+\left(\bar{\Gamma} * \bar{\Gamma}^{*}\right) * A^{-^{2}} \\
\quad+\left(\Gamma * \bar{\Gamma}^{*}\right) * A^{+} A^{-} * e^{i(\Delta \Phi+2 \theta)}+\left[\left(\Gamma * \bar{\Gamma}^{*}\right) * A^{+} A^{-} * e^{i(\Delta \Phi+2 \theta)}\right]^{*}
\end{aligned}
$$

Defining $z$ as:

$$
z \equiv\left(\Gamma * \bar{\Gamma}^{*}\right) * A^{+} A^{-} * e^{i(\Delta \Phi+2 \theta)}
$$

and using the identity $z+z^{*}=2 \mathcal{R}\{z\}$, yields:

$$
\begin{gathered}
I=\left(\Gamma * \Gamma^{*}\right) * A^{+^{2}}+\left(\bar{\Gamma} * \bar{\Gamma}^{*}\right) * A^{-^{2}}+\mathcal{R}\left\{\left(\Gamma * \bar{\Gamma}^{*}\right) * A^{+} A^{-} * e^{i(\Delta \Phi+2 \theta)}\right\} \\
=\left(\Gamma * \Gamma^{*}\right) * A^{+^{2}}+\left(\bar{\Gamma} * \bar{\Gamma}^{*}\right) * A^{-^{2}} \\
+\mathcal{R}\left\{\Gamma * \bar{\Gamma}^{*}\right\} * A^{+} A^{-} * 2 \cos (\Delta \Phi+2 \theta)
\end{gathered}
$$

where the the first two terms are the DC terms and the third term contains the convolution of the cosine function and the amplitude expression $\mathcal{R}\left\{\Gamma * \bar{\Gamma}^{*}\right\} * A^{+} A^{-}$. It should also be noted that $\Gamma * \Gamma^{*}$ and $\bar{\Gamma} * \bar{\Gamma}^{*}$ are the auto-correlations of $\Gamma$ and $\bar{\Gamma}$ respectively and $\Gamma * \bar{\Gamma}^{*}$ is the cross-correlation of $\Gamma$ and $\bar{\Gamma}$. 
Appendix C

MATLAB Scripts for the Numerical Simulations 


\section{C.1 Ideal DIC}

\% This code creates a semi-circle shaped phase-only object, takes

$\%$ its Fourier transform and then it multiplies it by an SLM

$\%$ function that is the sum of two phase ramps expressed by two

\% complex exponentionals (Euler's formula) that form an amplitude

$\%$ cosine pattern. Finally a fourier transform of multiplied $\%$ expression is taken to represent the CCD captured fields.

clc; clear all; close all

$\%=$ Coordinate Generation $=$

$\mathrm{a}=1 ; \%$ Side length of the object's matrix

$\mathrm{N}=512 ; \%$ Number of pixels

$\mathrm{dx}=\mathrm{a} / \mathrm{N}$

$\mathrm{x}=-\mathrm{a} / 2: \mathrm{dx}: \mathrm{a} / 2-\mathrm{dx} ;$

$\mathrm{y}=-\mathrm{a} / 2: \mathrm{dx}: \mathrm{a} / 2-\mathrm{dx} ;$

$[\mathrm{xx}, \mathrm{yy}]=$ meshgrid $(\mathrm{x}, \mathrm{y}) ; \% 2 \mathrm{D} \mathrm{x}$ and $\mathrm{y}$ corrdinate matrices

$\mathrm{fs}_{\mathrm{s}}=\mathrm{N} / \mathrm{a} ; \% 1 / \mathrm{dx}$

$\mathrm{df}=\mathrm{fs} / \mathrm{N}$

$f x=\operatorname{linspace}(-f s / 2$, fs $/ 2-d f, N)$;

$[f f x, f f y]=$ meshgrid $\left(f_{x}, f_{x}\right) ; \% 2 D f_{-} x$ and $f_{-} y$ matrices 
$\%=$ Creating the phase object $=$

$\mathrm{R}=1 / 4 * \mathrm{a} ; \%$ Radius is $1 / 4$ of the $\mathrm{X}$-coordinate dimension kappa $=1 / 3 ; \%$ Object's phase weakness factor

Phi_obj $=\operatorname{kappa} * 2 *$ pi*real $\left(\operatorname{sqrt}\left(\mathrm{R}^{\wedge} 2-\left(\mathrm{xx} \cdot{ }^{\wedge} 2+\mathrm{yy} \cdot{ }^{\wedge} 2\right)\right)\right) \cdot / \mathrm{R}$;

E_obj $=\exp (1 \mathrm{i} *$ Phi_obj $) ; \%$ Object's E field matrix

E_OBJ $=$ fftshift $\left(\right.$ fft $2\left(E_{-}\right.$obj $\left.) /\left(\mathrm{N}^{\wedge} 2\right)\right) ; \%$ FT of the object's

field matrix

$\%=$ SLM Function Parameters $=$

alpha $=0 ; \%$ Pattern rotation angle

$f f x_{-} t=f f x_{*} * \cos ($ alpha $)+f f y \cdot * \sin ($ alpha $) ; \%$ Rotated $f_{-} x$ coordinates

$f_{f} y_{-} t=f x_{-} \cdot * \sin ($ alpha $)-f f f_{-} * \cos ($ alpha $) ; \%$ Rotated $f_{-} y$ coordinates

$\mathrm{s}=1 * \mathrm{a} / \mathrm{N} ; \%$ Half shear distance

$\mathrm{s}_{-} \mathrm{x}=\mathrm{s} * \cos ($ alpha $)$

$\mathrm{s}_{-} \mathrm{y}=\mathrm{s} * \sin ($ alpha $)$;

$\%=$ Four-step PSI loop $=$

theta_j $=\left[\begin{array}{llll}0 & \text { pi } / 4 & \text { pi } / 2 & 3 * p i / 4\end{array}\right]$;

for $\mathrm{j}=1: 4$

\% SLM Function

$\operatorname{SLM}(:,:, j)=1 / 2 * \exp \left(1 \mathrm{i} *\left(2 * \mathrm{pi} *\left(\mathrm{~s}_{-} \mathrm{x} * \mathrm{ff} \mathrm{x}_{-} \mathrm{t}+\mathrm{s}_{-} \mathrm{y} * \mathrm{ffy} \mathrm{y}_{-} \mathrm{t}\right)+\right.\right.$ theta $\left.\left.\mathrm{j}_{-}(\mathrm{j})\right)\right) \ldots$

$+1 / 2 * \exp \left(-1 \mathrm{i} *\left(2 * \mathrm{pi} *\left(\mathrm{~s}_{-} \mathrm{x} * \mathrm{ff} \mathrm{x}_{-} \mathrm{t}+\mathrm{s}_{-} \mathrm{y} * \mathrm{ff} \mathrm{y}_{-} \mathrm{t}\right)+\mathrm{theta}_{-} \mathrm{j}(\mathrm{j})\right)\right)$ 
$\%$ Modulated field

$\mathrm{E} \_\mathrm{OUT}=\mathrm{E} \_$OBJ.$* \operatorname{SLM}(:,:, \mathrm{j})$;

\% Output fields at the detector plane

E_out $=$ fft $2($ E_OUT $) ;$

$\%$ Intensity

$\mathrm{I}(:,:, \mathrm{j})=($ E_out $* * \operatorname{conj}($ E_out $)) ;$

end

$\mathrm{I} 1=\mathrm{I}(:,:, 1) ;$

$\mathrm{I} 2=\mathrm{I}(:,:, 2)$;

$\mathrm{I} 3=\mathrm{I}(:,:, 3)$;

$\mathrm{I} 4=\mathrm{I}(:,:, 4)$;

$\%$ Plot the results $=$

\% Intensities from the four-step PSI

figure

subplot 221

imagesc $(\mathrm{x}, \mathrm{y}, \mathrm{I}(:,:, 1))$

title ('\theta $=0$ ', 'FontSize', 13)

xlabel('X-Axis', 'FontSize', 11)

ylabel ('Y-Axis', 'FontSize',11)

axis square

colormap gray 
colorbar

subplot 222

imagesc $(\mathrm{x}, \mathrm{y}, \mathrm{I}(:,:, 2))$

title ('\theta $=\backslash$ pi /4',' FontSize',13)

xlabel ('X-Axis', 'FontSize', 11)

ylabel ('Y-Axis', 'FontSize',11)

axis square

colormap gray

colorbar

subplot 223

imagesc $(x, y, I(:,:, 3))$

title ('\theta $=\backslash$ pi $/ 2$ ', 'FontSize',13)

xlabel ('X-Axis', 'FontSize',11)

ylabel ('Y-Axis', 'FontSize',11)

axis square

colormap gray

colorbar

subplot 224

imagesc $(\mathrm{x}, \mathrm{y}, \mathrm{I}(:,:, 4))$

title ('\theta $=3 \backslash \mathrm{pi} / 4$ ', 'FontSize', 13 )

xlabel ('X-Axis', 'FontSize', 11)

ylabel ('Y-Axis', 'FontSize',11)

axis square

colormap gray

colorbar 
$\%$ Plot Delta_Phi

figure

Delta_Phi $=\operatorname{atan}((\mathrm{I}(:,:, 4)-\mathrm{I}(:,:, 2)) \cdot /(\mathrm{I}(:,:, 1)-\mathrm{I}(:,:, 3)))$;

imagesc ( $\mathrm{x}, \mathrm{y}$, Delta_Phi $)$

xlabel ('X-Axis', 'FontSize', 13)

ylabel ('Y-Axis', 'FontSize', 13)

colorbar

set (gca, 'FontSize', 12)

$\mathrm{h}=$ colorbar

title(h,'`Delta $\backslash$ Phi - Rad.', 'FontSize',13)

\% compare cross section with the ideal case

$\mathrm{a}=$ Phi_obj $(512 / 2,3:$ end $)$;

$\mathrm{a}=\left[\begin{array}{lll}0 & \mathrm{a} & 0\end{array}\right]$

$\mathrm{b}=$ Phi_obj $(512 / 2,1$ : end - 2$)$;

$\mathrm{b}=\left[\begin{array}{lll}0 & \mathrm{~b} & 0\end{array}\right]$;

figure

$\mathrm{c}=\mathrm{b}-\mathrm{a} ;$

plot (x (1:4:end), c (1:4:end), 'xr', 'LineWidth', 1.5);

hold on; plot (x, Delta_Phi (512/2,:), 'LineWidth',2)

xlabel ('X-Axis', 'FontSize', 13)

ylabel(' $\backslash$ Delta $\backslash$ Phi - Rad. ', 'FontSize',13)

set (gca, 'FontSize',12) 


\section{C.2 Cosine Transmittance}

$\%$ This code creates a semi-circle shaped phase-only object, takes

$\%$ its Fourier transform and then it multiplies it by an SLM

$\%$ function that is the sum of two phase ramps expressed by two

\% complex exponentionals (Euler's formula) an a DC term that form

$\%$ an amplitude cosine pattern witha positive offset to simulate a

$\%$ cosine transittance function

\% Finally a fourier transform of multiplied expression is taken to

$\%$ represent the CCD captured fields.

clc; clear all; close all

$\%=$ Coordinate Generation $=$

$\mathrm{a}=1 ; \%$ Side length of the object's matrix

$\mathrm{N}=512 ; \%$ Number of pixels

$\mathrm{dx}=\mathrm{a} / \mathrm{N}$

$\mathrm{x}=-\mathrm{a} / 2: \mathrm{dx}: \mathrm{a} / 2-\mathrm{dx} ;$

$y=-a / 2: d x: a / 2-d x$

$[\mathrm{xx}, \mathrm{yy}]=$ meshgrid $(\mathrm{x}, \mathrm{y}) ; \% 2 \mathrm{D} \mathrm{x}$ and $\mathrm{y}$ corrdinate matrices

$\mathrm{fs}=\mathrm{N} / \mathrm{a} ; \% 1 / \mathrm{dx}$

$\mathrm{df}=\mathrm{fs} / \mathrm{N}$; 


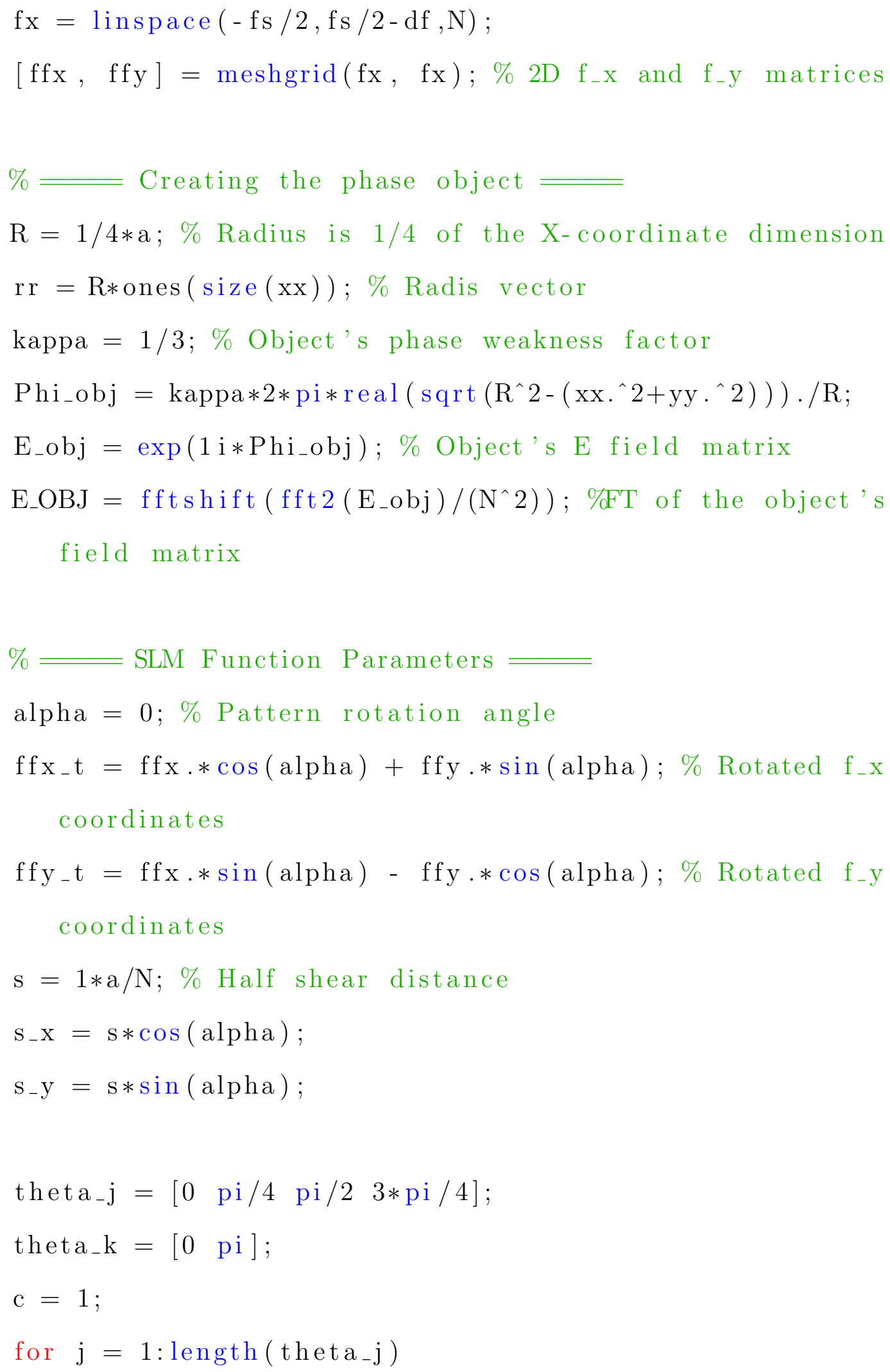




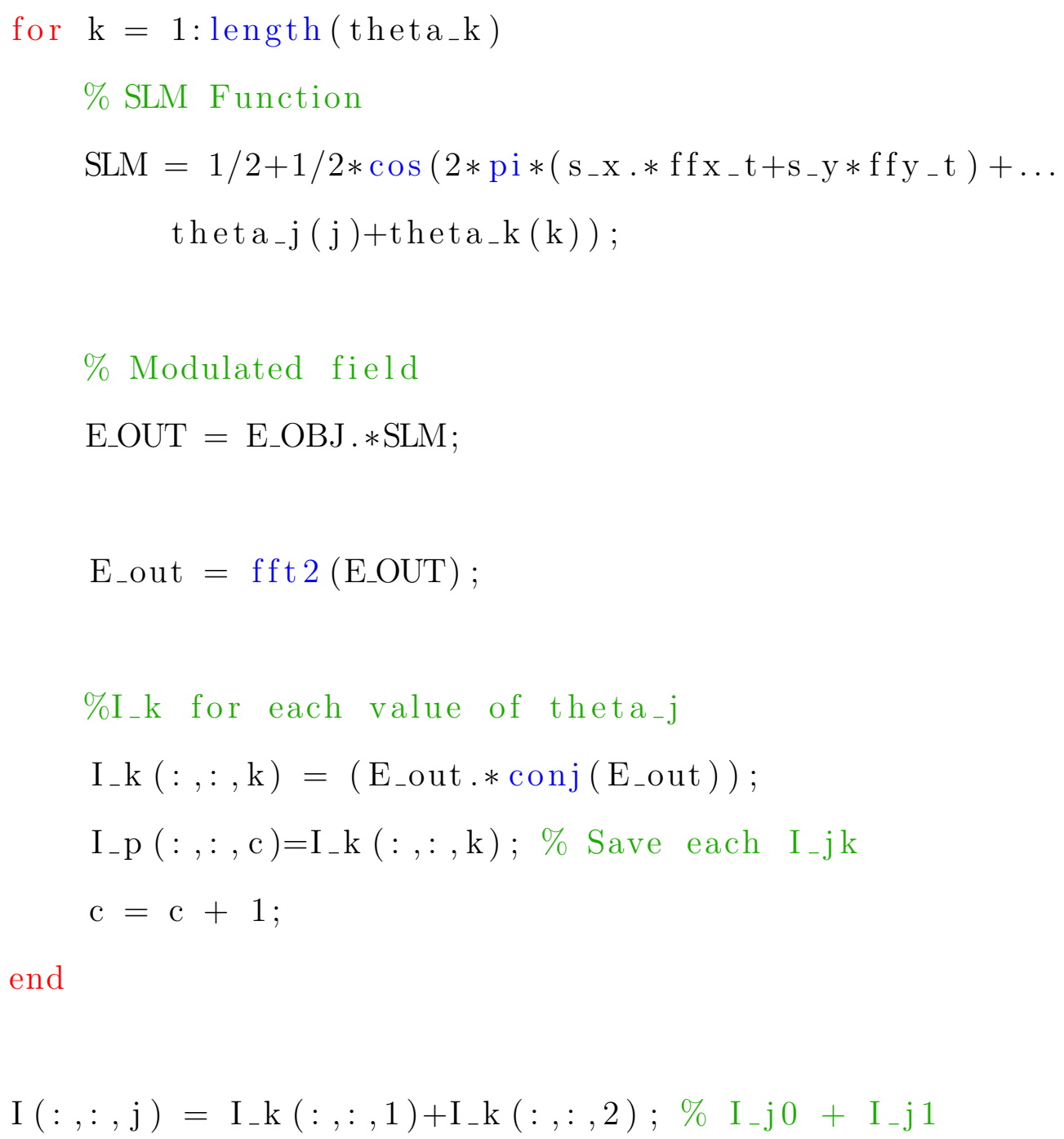

$$
\begin{aligned}
& \mathrm{I} 1=\mathrm{I}(:,:, 1) ; \\
& \mathrm{I} 2=\mathrm{I}(:,:, 2) ; \\
& \mathrm{I} 3=\mathrm{I}(:,:, 3) ; \\
& \mathrm{I} 4=\mathrm{I}(:,:, 4) ;
\end{aligned}
$$

$\%$ Plot the results

$\%$ Intensities from the eight-step PSI 
figure

subplot 421

$\operatorname{imagesc}\left(\mathrm{x}, \mathrm{y}, \mathrm{I}_{-} \mathrm{p}(:,:, 1)\right)$

title (' $\backslash$ theta ${ }_{-} \mathrm{j}=0, \backslash$ theta $\mathrm{k}=0$ ', 'FontSize',12)

xlabel ('X-Axis', 'FontSize', 10)

ylabel ('Y-Axis', 'FontSize', 10)

axis square

colormap gray

colorbar

subplot 422

$\operatorname{imagesc}\left(\mathrm{x}, \mathrm{y}, \mathrm{I}_{-} \mathrm{p}(:,:, 2)\right)$

title ('\theta ${ }_{-}=0, \backslash$ theta $k=\backslash p i$ ', 'FontSize',12)

xlabel ('X-Axis', 'FontSize', 10)

ylabel ('Y-Axis', 'FontSize',10)

axis square

colormap gray

colorbar

subplot 423

$\operatorname{imagesc}\left(\mathrm{x}, \mathrm{y}, \mathrm{I}_{-} \mathrm{p}(:,:, 3)\right)$

title ('\theta $\mathrm{j}_{-}=\backslash \mathrm{pi} / 4, \backslash$ theta_k =0',' FontSize', 12)

xlabel ('X-Axis', 'FontSize', 10)

ylabel ('Y-Axis', 'FontSize',10)

axis square

colormap gray

colorbar

subplot 424 


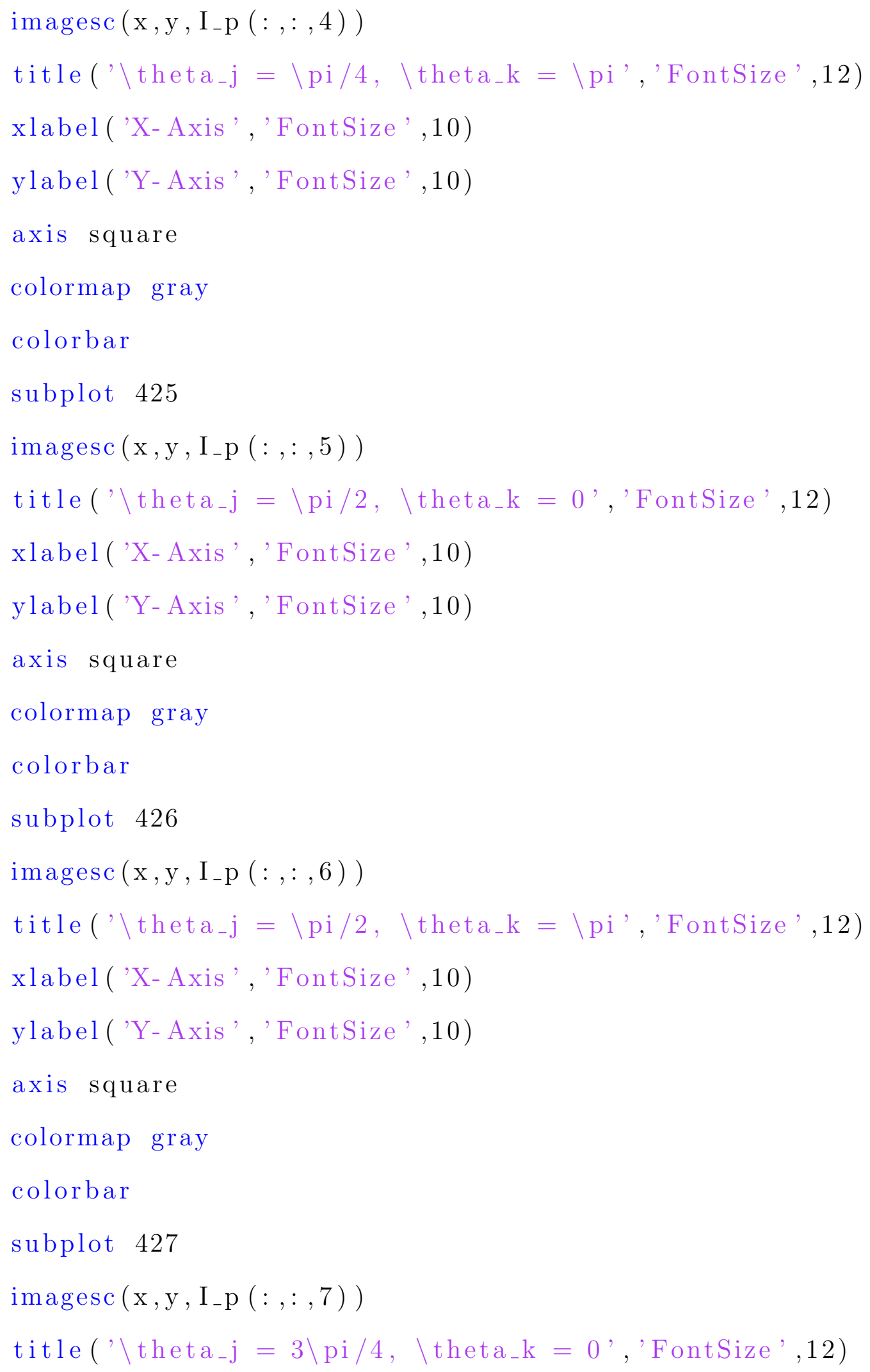




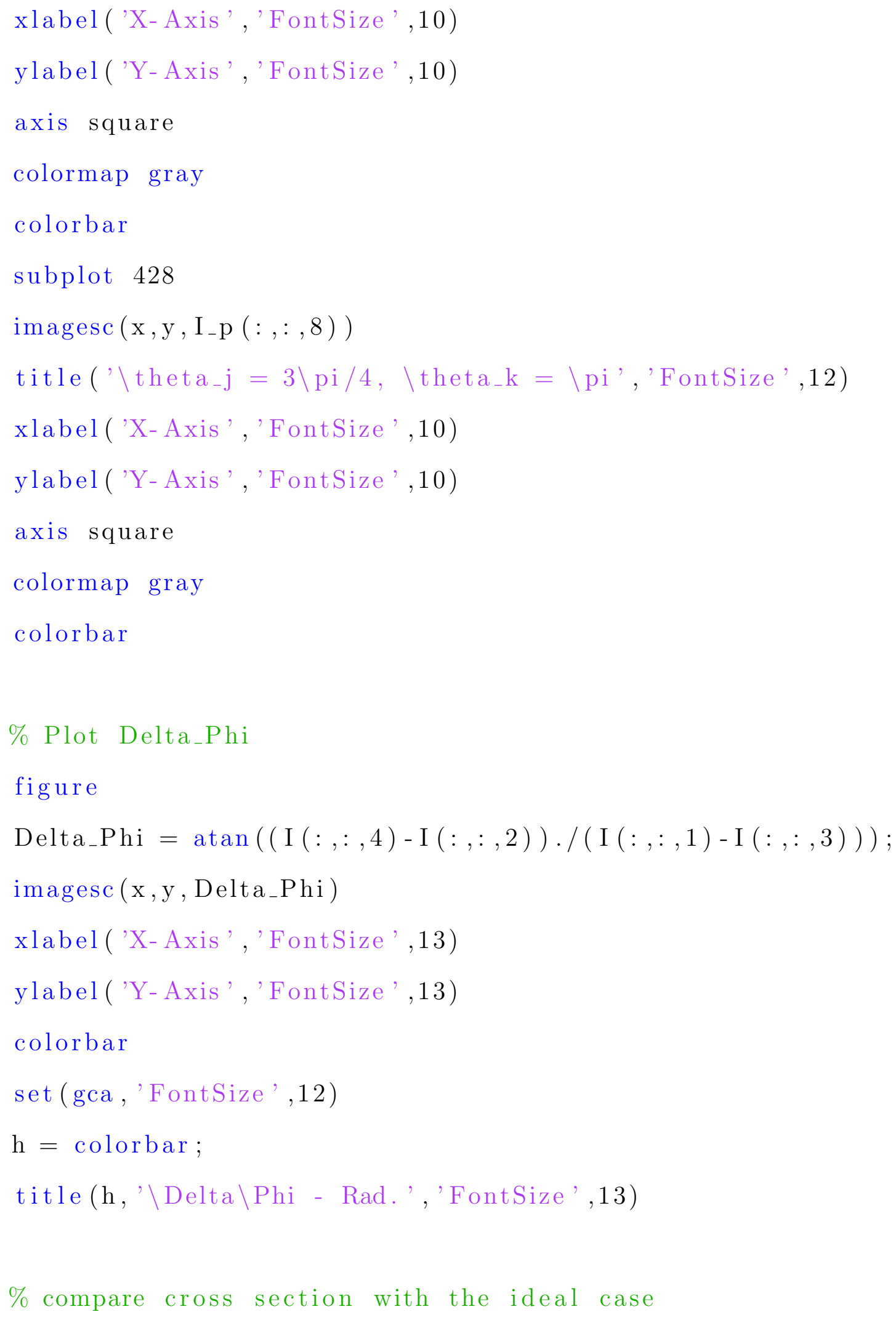




$$
\begin{aligned}
& \mathrm{a}=\text { Phi_obj }(\mathrm{N} / 2,3 \text { : end }) ; \\
& \mathrm{a}=\left[\begin{array}{lll}
0 & \mathrm{a} & 0
\end{array}\right] ; \\
& \mathrm{b}=\text { Phi_obj }(\mathrm{N} / 2,1 \text { : end }-2) ; \\
& \mathrm{b}=\left[\begin{array}{lll}
0 & \mathrm{~b} & 0
\end{array}\right] ; \\
& \mathrm{c}=\mathrm{b}-\mathrm{a} ;
\end{aligned}
$$

figure

$\operatorname{plot}(\mathrm{x}(1: 4:$ end $), \mathrm{c}(1: 4:$ end $)$, 'xr' , 'LineWidth', 1.5);

hold on; plot (x, Delta_Phi (512/2,:), 'LineWidth',2)

xlabel ('X-Axis', 'FontSize', 13)

ylabel ('\Delta \Phi - Rad.', 'FontSize',13)

set (gca,' FontSize', 12) 


\section{C.3 Randomly Combined Ramps}

\% This code creates a semi-circle shaped phase-only object, takes

$\%$ its Fourier transform and then it multiplies it by two SLM'

S

$\%$ functions which are randomly interleaved phase-only ramps.

\% Finally a fourier transform of multiplied expression is taken to

$\%$ represent the CCD captured fields.

clc; clear all; close all

$\%=$ Coordinate Generation $=$

$\mathrm{a}=1 ; \%$ Side length of the object's matrix

$\mathrm{N}=512 ; \%$ Number of pixels

$\mathrm{dx}=\mathrm{a} / \mathrm{N}$

$x=-a / 2: d x: a / 2-d x ;$

$y=-a / 2: d x: a / 2-d x ;$

$[\mathrm{xx}, \mathrm{yy}]=$ meshgrid $(\mathrm{x}, \mathrm{y}) ; \% 2 \mathrm{D} \mathrm{x}$ and $\mathrm{y}$ corrdinate matrices

$\mathrm{fs}=\mathrm{N} / \mathrm{a} ; \% 1 / \mathrm{dx}$

$\mathrm{df}=\mathrm{fs} / \mathrm{N}$

$\mathrm{fx}=\operatorname{linspace}(-\mathrm{fs} / 2, \mathrm{fs} / 2-\mathrm{df}, \mathrm{N})$;

$[\mathrm{ffx}, \mathrm{ffy}]=\operatorname{meshgrid}(\mathrm{fx}, \mathrm{fx}) ; \% 2 \mathrm{D} \mathrm{f}_{-} \mathrm{x}$ and $\mathrm{f}_{-} \mathrm{y}$ matrices

$\%=$ Creating the phase object $=$ 
$\mathrm{R}=1 / 4 * \mathrm{a} ; \%$ Radius is $1 / 4$ of the $\mathrm{X}$-coordinate dimension kappa $=1 / 3 ; \%$ Object's phase weakness factor

Phi_obj $=\operatorname{kappa} * 2 *$ pi $*$ real $\left(\operatorname{sqrt}\left(\mathrm{R}^{\wedge} 2-\left(\mathrm{xx} \cdot{ }^{\wedge} 2+\mathrm{yy} \cdot{ }^{\wedge} 2\right)\right)\right) \cdot / \mathrm{R}$;

E_obj $=\exp (1 \mathrm{i} *$ Phi_obj $) ; \%$ Object's E field matrix

E_OBJ $=$ fftshift $\left(\right.$ fft2 $\left.\left(\mathrm{E}_{-} \mathrm{obj}\right) /\left(\mathrm{N}^{\wedge} 2\right)\right)$; \%FT of the object's

field matrix

$\%=$ SLM Function $=$

alpha $=0 ; \%$ Pattern rotation angle

$f f x_{-} t=f f x_{*} * \cos ($ alpha $)+f f y \cdot * \sin ($ alpha $) ; \%$ Rotated $f_{-} x$

coordinates

$f y_{-} t=f f x \cdot * \sin ($ alpha $)-f f y \cdot * \cos ($ alpha $) ; \%$ Rotated $f_{-} y$

coordinates

$\mathrm{s}=1 * \mathrm{a} / \mathrm{N} ; \%$ Half shear distance

$\mathrm{s}_{-} \mathrm{x}=\mathrm{s} * \cos ($ alpha $)$

$\mathrm{s}_{-} \mathrm{y}=\mathrm{s} * \sin ($ alpha $)$;

\% Random binary mask and its compliment

[rand_mask, rand_mask_hat] $=$ randomMask $(\mathrm{N}, \mathrm{N})$;

$\%=$ Eight-step PSI loop $=$

theta_j $=\left[\begin{array}{llll}0 & \text { pi } / 4 & \text { pi } / 2 & 3 * p i / 4\end{array}\right]$;

theta_k $=\left[\begin{array}{ll}0 & \mathrm{pi}\end{array}\right]$;

$\mathrm{c}=1 ; \%$ Counter variable

for $j=1: \operatorname{length}\left(\right.$ thet $\left._{-} \mathbf{j}\right)$

for $\mathrm{k}=1$ : length (theta_k)

\% Ramp patterns set 


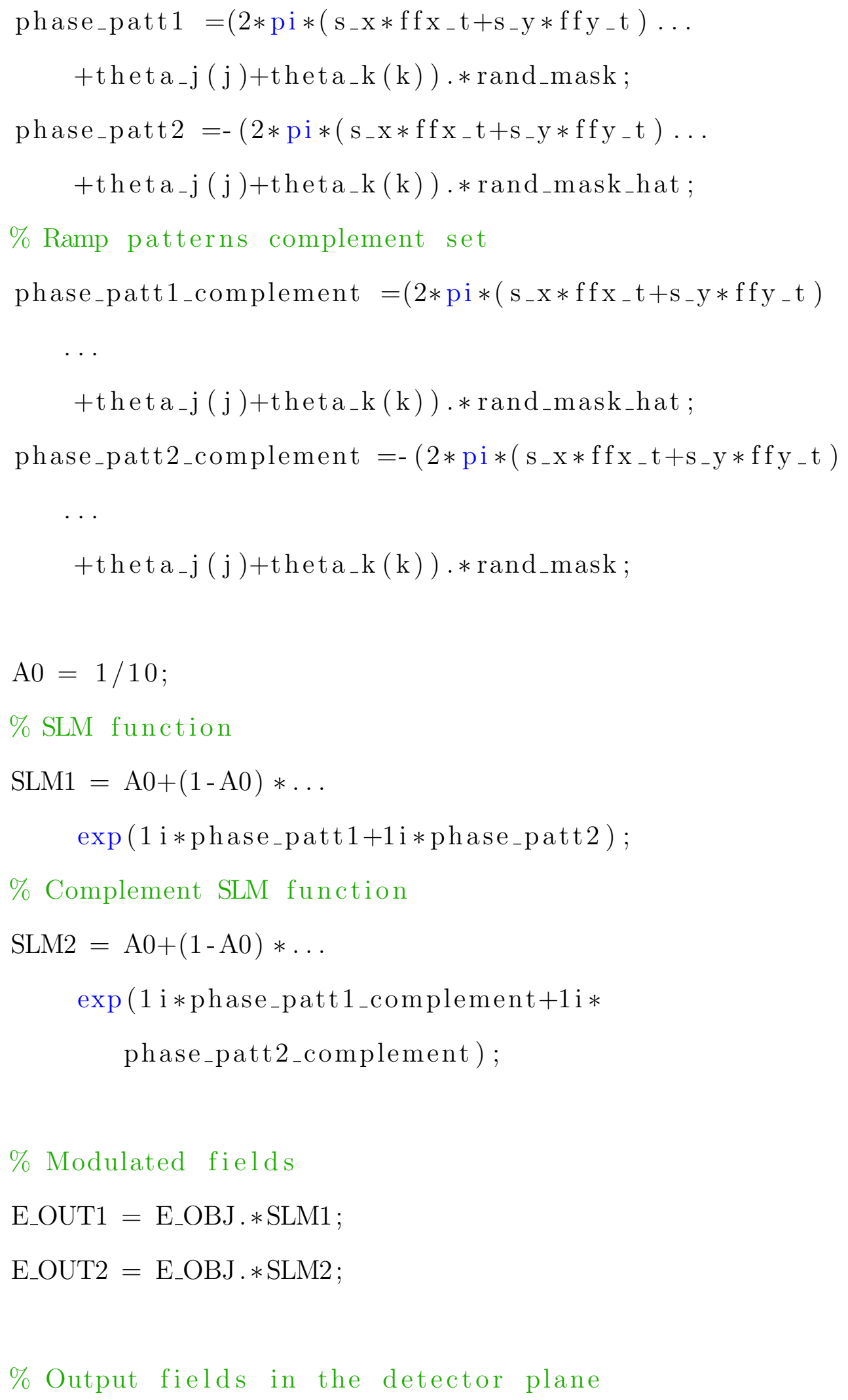




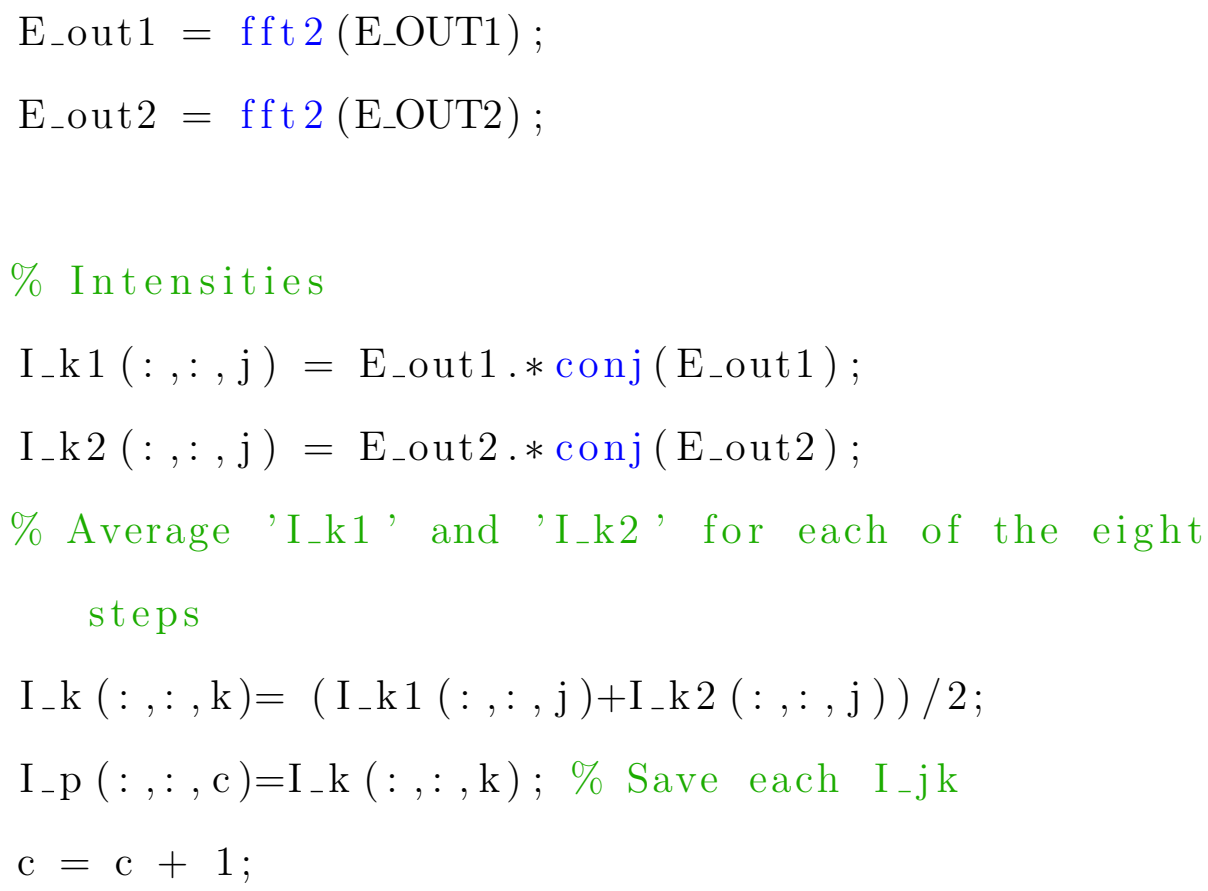

end

$$
\mathrm{I}_{-} \mathrm{j}(:,:, \mathrm{j})=\mathrm{I}_{-} \mathrm{k}(:,:, 1)+\mathrm{I}_{-} \mathrm{k}(:,:, 2) ;
$$

end

$\%$ Plot the results

$\%$ Intensities from the eight-step PSI

figure

subplot 421

$\operatorname{imagesc}\left(\mathrm{x}, \mathrm{y}, \mathrm{I}_{-} \mathrm{p}(:,:, 1)\right)$

title (' $\backslash$ theta ${ }_{-} \mathrm{j}=0, \backslash$ theta $\mathrm{k}=0$ ', 'FontSize', 12 )

xlabel ('X-Axis' ,'FontSize',10)

ylabel ('Y-Axis', 'FontSize',10)

axis square

colormap gray

colorbar 
subplot 422

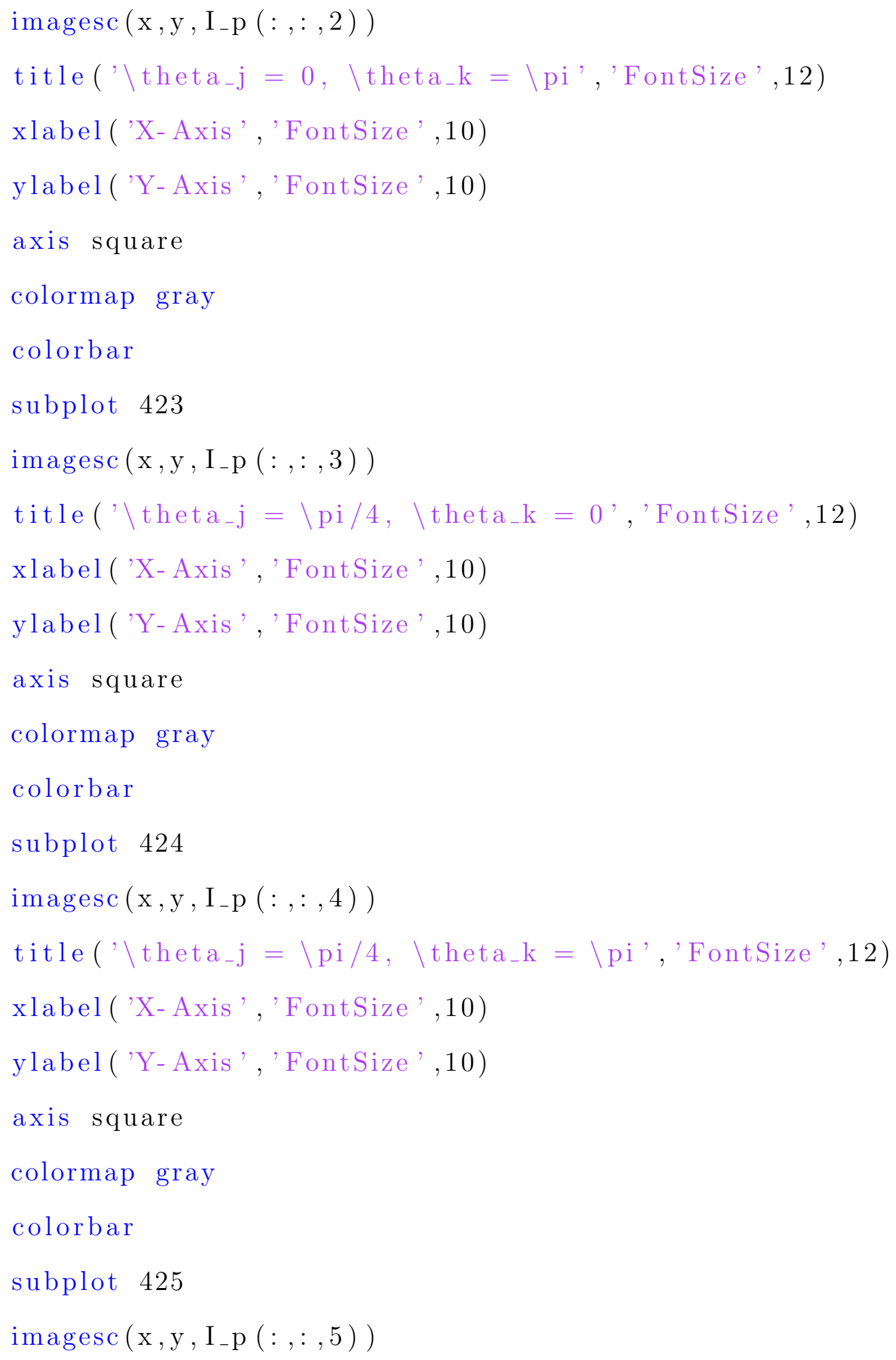




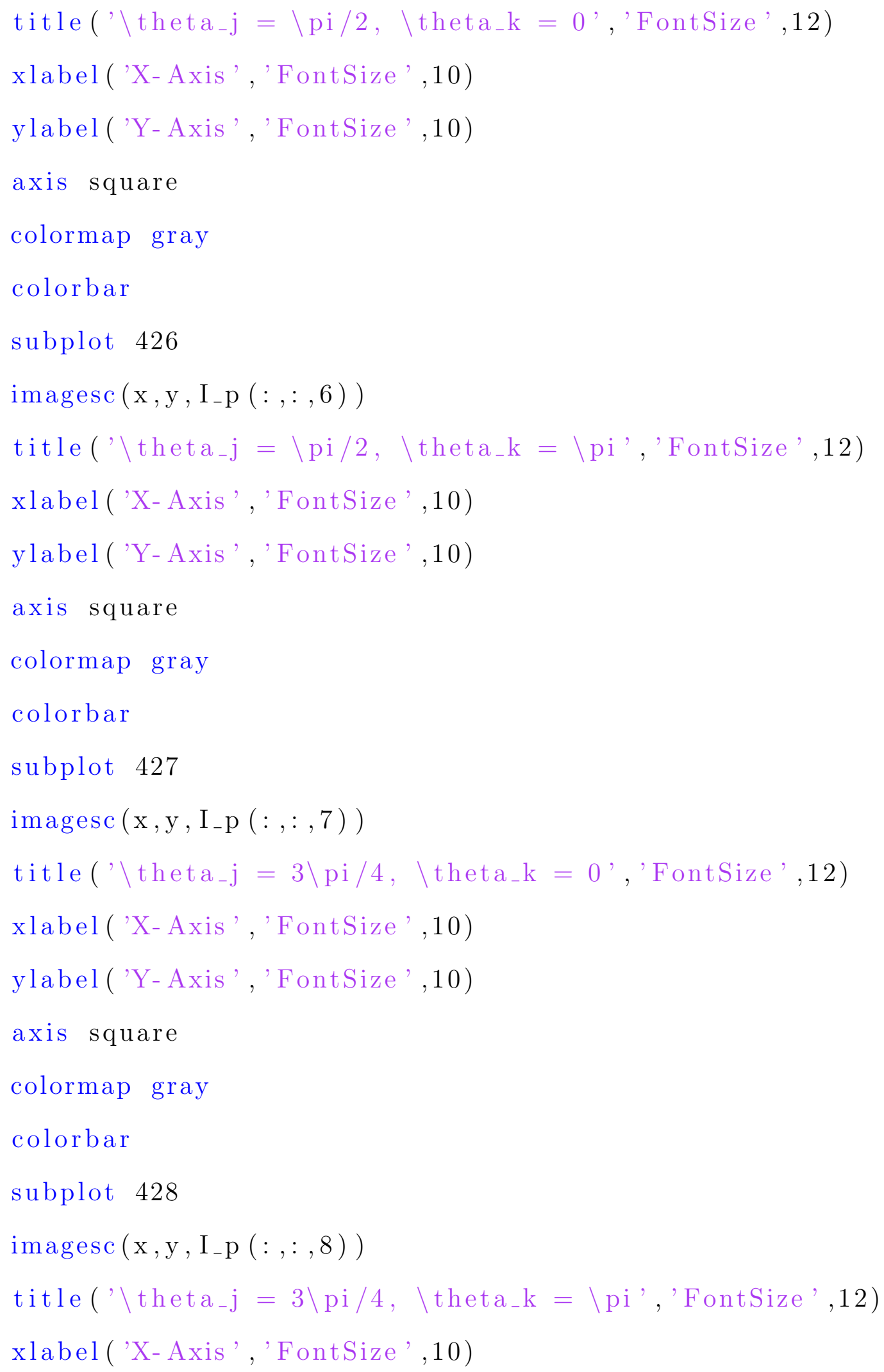


ylabel ('Y-Axis', 'FontSize', 10)

axis square

colormap gray

colorbar

\% Calculate object's phase gradient (Delta_Phi)

Delta_Phi $=\operatorname{atan}\left(\left(\mathrm{I}_{-} \mathrm{j}(:,:, 4)-\mathrm{I}_{-} \mathrm{j}(:,:, 2)\right) \ldots\right.$

$$
\left.. /\left(I_{-} \mathrm{j}(:,:, 1)-\mathrm{I}_{-} \mathrm{j}(:,:, 3)\right)\right) \text {; }
$$

$\%$ Plot Delta_Phi

figure

imagesc ( $x, y$, Delta_Phi $)$

xlabel ('X-Axis', 'FontSize', 13)

ylabel ('Y-Axis', 'FontSize', 13)

colorbar

set (gca, 'FontSize', 12)

$\mathrm{h}=$ colorbar;

title(h,'\Delta \Phi - Rad.', 'FontSize',13)

\% compare cross section with the ideal case

$\mathrm{a}=$ Phi_obj $(\mathrm{N} / 2,3$ : end $)$;

$\mathrm{a}=\left[\begin{array}{lll}0 & \mathrm{a} & 0\end{array}\right]$;

$\mathrm{b}=$ Phi_obj $(\mathrm{N} / 2,1$ : end -2$)$;

$\mathrm{b}=\left[\begin{array}{lll}0 & \mathrm{~b} & 0\end{array}\right]$; 
$\mathrm{c}=\mathrm{b}-\mathrm{a} ;$

figure

plot (x (1:4:end), c (1:4:end), 'r' ,'LineWidth', 1.5);

hold on; plot (x, Delta_Phi $(512 / 2,:)$, 'LineWidth',2)

xlabel ('X-Axis', 'FontSize',13)

ylabel ('\Delta $\backslash$ Phi - Rad. ', 'FontSize', 13)

set (gca,' 'FontSize', 12) 


\section{C.4 Random Binary Mask Generator Function}

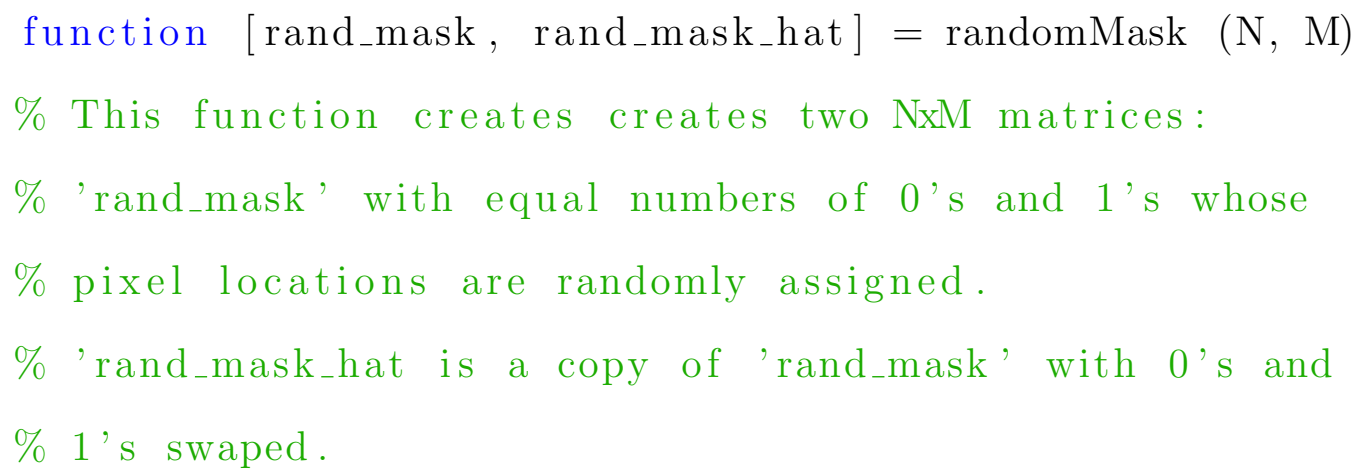

randomIndex $=\operatorname{randperm}(\mathrm{M} * \mathrm{~N},(\mathrm{M} * \mathrm{~N}) / 2)$;

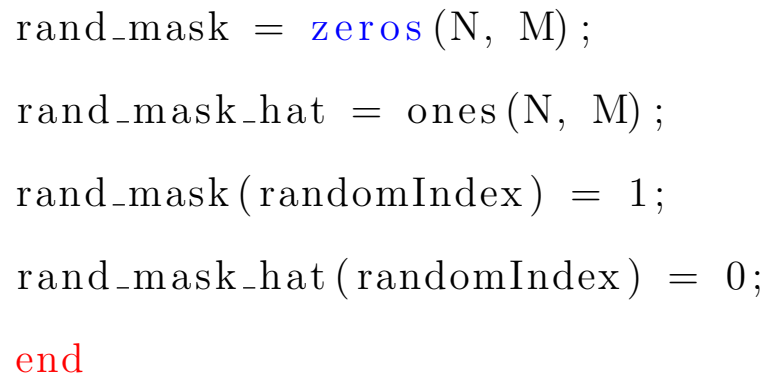

